\title{
TOXIC VOLATILE ORGANIC COMPOUNDS IN ENVIRONMENTAL TOBACCO SMOKE: EMISSION FACTORS FOR MODELING EXPOSURES OF CALIFORNIA POPULATIONS
}

Prepared by:

J.M. Daisey, K.R.R. Mahanama and A.T. Hodgson

Indoor Environment Program

Energy and Environment Division

Lawrence Berkeley Laboratory

University of California

Berkeley, CA 94720

510/486-7491

FAX 510/486-6658

Prepared for:

California Air Resources Board

2020 L Street

P.O. Box 2815

Sacramento, CA 95814

October 1994

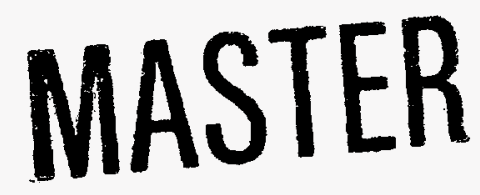

This report was submitted in fulfillment of ARB Contract Number A133-186, by Lawrence Berkeley Laboratory under the sponsorship of the California Air Resources Board and the Director, Office of Energy Research, Office of Health and Environmental Research, Human Health and Assessments Division, U.S. Department of Energy under Contract No. DE-AC03-76SF00098.

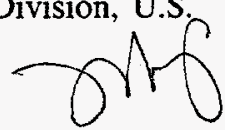




\section{DISCLAIMER}

The statements and conclusions in this report are those of the contractor and not necessarily those of the California Air Resources Board. The mention of commercial products, their source or their use in connection with material reported herein is not to be construed as either actual or implied endorsement of such products.

\section{ACKNOWLEDGMENTS}

The authors wish to thank the following for their generous advice and assistance in this project: Dr. K. Brunnemann, American Health Foundation, for providing information on the sidestream smoking apparatus; Dr. Chortyk, U.S. Dept. of Agriculture, Tobacco Safety Research Unit, Agricultural Research Service, for providing information on the manufacture of cigarettes and methods used to determine the weight of combusted tobacco; $\mathrm{H}$. Johnson, California Air Resources Board, for providing the data in Table 1 which were generated using weighted frequencies from a data tape provided by the Tobacco Control Section of the Department of Health Services, and Thu Phan, Lawrence Berkeley Laboratory, for her assistance in the analysis of some of the samples.

We also particularly wish to thank Elizabeth Ota, California Air Resources Board, for her advice and assistance during this project and her many constructive editorial suggestions during the preparation of this report.

This report was submitted in fulfillment of ARB Contract Number A133-186, "Toxic Volatile Organic Compounds in Environmental Tobacco Smoke: Emission Factors for Modeling Exposures of California Populations," by Lawrence Berkeley Laboratory under the sponsorship of the California Air Resources Board and the Birector, Office of Energy Research, Office of Health and Environmental Research, Human Health and Assessments Division, U.S. Department of Energy under Contract No. DE-AC03-76SF00098. Work was completed as of May, 1994. 


\section{DISCLAIMER}

This report was prepared as an account of work sponsored by an agency of the United States Government. Neither the United States Government nor any agency thereof, nor any of their employees, make any warranty, express or implied, or assumes any legal liability or responsibility for the accuracy, completeness, or usefulness of any information, apparatus, product, or process disclosed, or represents that its use would not infringe privately owned rights. Reference herein to any specific commercial product, process, or service by trade name, trademark, manufacturer, or otherwise does not necessarily constitute or imply its endorsement, recommendation, or favoring by the United States Government or any agency thereof. The views and opinions of authors expressed herein do not necessarily state or reflect those of the United States Government or any agency thereof. 


\section{DISCLAIMER}

Portions of this document may be illegible in electronic image products. Images are produced from the best available original document. 


\begin{abstract}
The primary objective of this study was to measure emission factors for selected toxic air contaminants in environmental tobacco smoke (ETS) using a room-sized environmental chamber. The emissions of 23 volatile organic compounds (VOCs), including 1,3-butadiene, three aldehydes and two vapor-phase $\mathrm{N}$-nitrosamines were determined for six commercial brands of cigarettes and reference cigarette 1R4F. The commercial brands were selected to represent $62.5 \%$ of the cigarettes smoked in California. For each brand, three cigarettes were machine smoked in the chamber. The experiments were conducted over four hours to investigate the effects of aging. Emission factors of the target compounds were also determined for sidestream smoke (SS).

For almost all target compounds, the ETS emission factors were significantly higher than the corresponding SS values probably due to less favorable combustion conditions and wall losses in the SS apparatus. Where valid comparisons could be made, the ETS emission factors were generally in good agreement with the literature. Therefore, the ETS emission factors, rather than the SS values, are recommended for use in models to estimate population exposures from this source. The variabilities in the emission factors $(\mu \mathrm{g} /$ cigarette $)$ of the selected toxic air contaminants among brands, expressed as coefficients of variation, were 16 to $29 \%$. Therefore, emissions among brands were generally similar. Differences among brands were related to the smoked lengths of the cigarettes and the masses of consumed tobacco. Mentholation and whether a cigarette was classified as light or regular did not significantly affect emissions. Aging was determined not to be a significant factor for the target compounds. There were, however, deposition losses of the less volatile compounds to chamber surfaces.
\end{abstract}




\section{TABLE OF CONTENTS}

DISCLAIMER -

ACKNOWLEDGMENTS - ii

ABSTRACT - iv

LIST OF TABLES -._-_ vii

LIST OF FIGURES -

1.0 SUMMARY AND CONCLUSIONS 1

1.1 Objectives and study design -.._. 1

1.2 Determination of the ETS emission factors ( $\mu \mathrm{g} /$ cigarette) for selected toxic air contaminants in a room-sized environmental chamber under conditions which simulate typical indoor settings --.-_- 3

1.3. Estimation of the range and variability of the ETS emission factors among a subset of popular cigarettes brands which have large market shares in California -_-_--- 6

1.4. Determination of emission factors for the same VOCs in sidestream smoke for comparison with the ETS measurements --_-_ 6

1.5. Investigation of the effect of aging on the apparent emission factors of 1,3-butadiene and other VOCs in ETS - 7

2.0 RECOMMENDATIONS

2.1. Surface deposition and re-emission rates of VOCs --_._.

2.2. Improved sampling method for acrolein -

2.3. Tobacco-specific tracers for VOCs in ETS -

2.4. Loss mechanisms for nicotine -_- 12

2.5. Measurement of emission factors in buildings -

3.0 STATEMENT OF PROBLEM -...13

3.1 Background-13

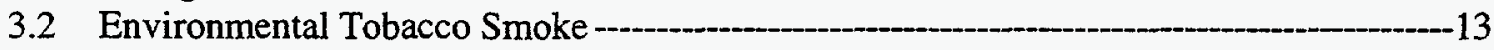

3.3 Limitations of Available Data on ETS -

4.0 OBJECTIVES -

5.0 STUDY DESIGN -

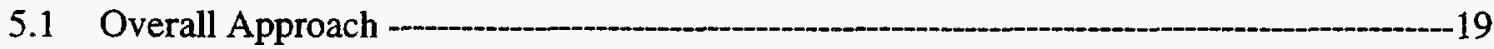

5.2 Cigarettes -

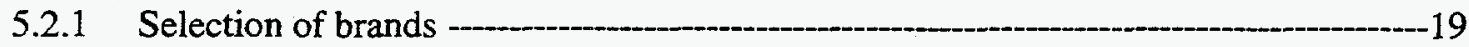

5.2.2 Protocols for Cigarettes and Smoking -_._._.

5.3 Environmental Chamber -

5.4 Protocols for the Environmental Chamber Experiments -.__

5.5 Apparatus and Experimental Protocols for Measurements of Sidestream Smoke -.--_-30

5.6 Sampling and Chemical Analysis for N-Nitrosamines in Tobacco Smoke -_-___-_-_31

5.6.1 Development of a Sampling and Analysis Method for N-Nitrosamines in Tobacco Smoke-_- 34

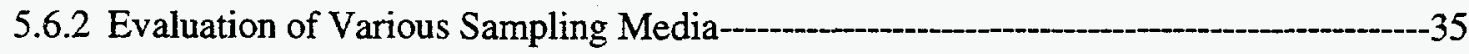

5.6.3 Development of a Sample Clean-up Method for VNA Collected on Thermosorb/N Cartridges -..-

5.6.4 Comparison of Thermosorb/N Cartridge \& Impinger Methods of Sample Collection -38 
5.7 Sampling and Chemical Analysis for Other Species-

5.7.1 Aldehydes -

5.7.2 Volatile Organic Compounds -

5.7.3 Nicotine---

5.7.4 Airbome Particulate Matter -

5.7.5 $\mathrm{NO}_{\mathrm{X}}$ and $\mathrm{CO}$

5.8 Data Reduction and Analysis -

5.8.1 Emission Factors for ETS-

5.8.2 Emission Factors for SS-_-50

5.8.3 Experimental Uncertainties in the Measurements -

5.8.4 Lower Limits of Detection-1-54

6.0 RESULTS AND DISCUSSION -

6.1 Emission Factors for Environmental Tobacco Smoke and Sidestream Smoke --_---57

6.1.1 Volatile N-Nitrosamines --._-

6.1.2 Aldehydes --62

6.1.3 Volatile Organic Compounds -

6.1.4 Nicotine--1-86

6.1.5 Particulate Matter--_-90

6.1.6 NO, NOx and CO-95

7.0 REFERENCES -

8.0 GLOSSARY OF TERMS - 104

APPENDIX 105 


\section{LIST OF TABLES}

1.1. Summary of environmental tobacco smoke emission factors determined for six commercial cigarettes and Kentucky reference cigarette $1 \mathrm{R} 4 \mathrm{~F}$.

1.2. Comparison of ETS and SS emission factors determined for six commercial brands of cigarettes, $\mu \mathrm{g} /$ cigarette.

3.1. Some VOCs and Aldehydes Reported in Cigarette Smoke which are on CARB's list of toxic air contaminants.

5.1. Brands of cigarettes purchased by California smokers over 18 years of age.

5.2. Brand preferences of various ethnic categories in California as percent citing.

5.3. Cigarette dimensions and tobacco weights per unit length.

5.4. Average temperatures and relative humidities for the environmental chamber experiments.

5.5. Summary of the sampling protocols and analysis methods for ETS measurements.

5.6. Summary of the sampling protocols and analysis methods for SS measurements.

5.7. Percentage recovery of spiked VNA from different sampling media.

5.8. Comparison of two sampling methods for N-nitrosamines in ETS, ng/cigarette.

5.9. Relative uncertainties in the analytical response factors for the measured compounds.

5.10. Estimated relative uncertainties in ETS emission factors for the measured compounds, $\mu \mathrm{g} /$ cigarette.

5.11. Estimated relative uncertainties in SS emission factors for the measured compounds, $\mu \mathrm{g} /$ cigarette.

5.12. Estimated lower limits of detection for the compounds measured in environmental tobacco smoke and sidestream smoke.

6.1. Percentage distribution of VNA in sidestream smoke among cartridge, filter and sidestream apparatus.

6.2. Summary of VNA emission factors for sidestream smoke and environmental tobacco smoke from six commercial cigarettes and Kentucky reference cigarette 1R4F.

6.3. Comparison of SS emission factors for VNA reported for American, Swiss and German cigarettes and Kentucky reference cigarette 1R4F, ng/cigarette.

6.4. Summary of aldehyde emission factors for environmental tobacco smoke from six commercial cigarettes and Kentucky reference cigarette $1 \mathrm{R} 4 \mathrm{~F}$.

6.5. Summary of aldehyde emission factors for sidestream smoke from six commercial cigarettes and Kentucky Reference cigarette 1R4F. 
6.7. Comparison of ETS and SS aldehyde emission factors determined in this study to literature data.

6.8. Summary of VOC emission factors for environmental tobacco smoke from six commercial cigarettes and Kentucky reference cigarette 1R4F, ng/mg of tobacco consumed.

6.9. Summary of VOC emission factors for environmental tobacco smoke from six commercial cigarettes and Kentucky reference cigarette $1 R 4 F, \mu \mathrm{g} /$ cigarette.

6.10. Calculated $\mathrm{k}$ values for 3-vinylpyridine, phenol and cresols from six commercial cigarettes and Kentucky reference cigarette 1R4F.

6.11. Corrected and uncorrected ETS emission factors for 3-vinylpyridine, phenol and cresols from six commercial cigarettes and Kentucky reference cigarette 1R4F, $\mathrm{ng} / \mathrm{mg}$ tobacco consumed.

6.12. Corrected and uncorrected ETS emission factors for 3-vinylpyridine, phenol and cresols from six commercial cigarettes and Kentucky reference cigarette 1R4F, $\mu \mathrm{g} / \mathrm{cigarette}$.

6.13. Summary of VOC emission factors for sidestream smoke from six commercial cigarettes and Kentucky reference cigarette $1 \mathrm{R} 4 \mathrm{~F}, \mathrm{ng} / \mathrm{mg}$ of tobacco consumed.

6.14. Summary of VOC emission factors for sidestream smoke from six commercial cigarettes and Kentucky reference cigarette 1R4F, $\mu \mathrm{g} /$ cigarette.

6.15 Comparison of average ETS and SS emission Factors for eleven VOCs.

6.16. Comparison of the VOC emission factors determined in this study to literature data.

6.17. Summary of nicotine emission factors for environmental tobacco smoke and sidestream smoke from six commercial cigarettes and Kentucky reference cigarette 1R4F.

6.18. Comparison of nicotine emission factors determined in this study to literature data.

6.19. Summary of corrected and uncorrected ETS emission factors for PM-2.5 $5_{\mathrm{EQ}}$ for six commercial cigarettes and Kentucky reference cigarette 1R4F.

6.20. Summary of SS particulate matter emission factors for six commercial cigarettes and Kentucky reference cigarette 1R4F.

6.21. ETS particulate matter emission factors determined in environmental chambers.

6.22. Comparison of ratios of ETS particulate matter to ETS nicotine.

6.23 Environmental chamber concentrations of carbon monoxide and nitric oxide from environmental tobacco smoke during experiments with six commercial cigarettes and Kentucky reference cigarette 1R4F. 


\section{LIST OF FIGURES}

5.1 Schematic diagram showing a cross sectional view of the environmental chamber.

5.2 Schematic diagram of locations, configurations, and sampling rates for samples collected in the environmental chamber experiments. MFC = mass flow controller.

5.3 Schematic diagram of the sidestream apparatus. 30

5.4 Schematic diagrams of the sampling trains used for collecting $\mathrm{N}$-nitrosamines in a.) ETS and b.) SS.

5.5 Schematic diagram of the sampling trains for aldehydes and VOCs in SS.

5.6 Total-ion-current chromatogram of an ETS sample collected from the environmental chamber.

5.7 Schematic diagram of the sampling train for collection of nicotine and particulate matter in a.) ETS and b.) SS.

6.1 Variations in chamber concentrations of selected VOCs in ETS over time for cigarette B.

6.2 Plots of In measured concentration versus initial sampling time for 3-vinylpyridine in chamber experiments with six commercial cigarettes and reference cigarette $1 \mathrm{R} 4 \mathrm{~F}$. 


\subsection{SUMMARY AND CONCLUSIONS}

\subsection{Objectives and study design}

Hodgson and Wooley (1991) identified environmental tobacco smoke (ETS) as an important source of a number of toxic air contaminants in indoor air, including 1,3-butadiene, acetaldehyde, acrolein, $\mathrm{N}$-nitrosamines, xylenes, phenols, and cresols. Environmental tobacco smoke is the smoke to which nonsmokers are exposed when they are in an indoor environment with smokers. It is composed largely of sidestream tobacco smoke (SS), the smoke emitted by the smoldering end of a cigarette between puffs, with minor contributions from exhaled mainstream smoke (the smoke which is directly inhaled by the smoker) and any smoke that escapes from the burning part of the tobacco during puff-drawing by the smoker. ETS differs from SS in that it is highly diluted and dispersed within a room and it undergoes aging.

Recent research indicates that $61 \%$ of California adults are exposed to ETS for an average of about 5 hours per day (Wiley, et al., 1991; Jenkins, et al., 1992). Thus, exposures to toxic air contaminants from this source alone are widespread in California. Although ETS is likely to be the major source of exposure for many of these compounds, quantitative data to evaluate exposures to the population of California are often lacking or weak (Daisey, et al., 1991). In addition, emission factors for SS or ETS reported in the older literature may differ from emission factors for the cigarettes currently sold because of changes in cigarette manufacturing processes to reduce tar in mainstream smoke, e.g., more use of reconstituted tobacco.

The overall goal of this study was to provide up-to-date emission factors for selected volatile organic compounds (VOCs) in ETS which can be used in models to estimate exposures of the California population. Most of these VOCs have been designated toxic air contaminants by the State of California. The specific objectives were to:

1. Determine the ETS emission factors ( $\mu \mathrm{g} /$ cigarette) for selected toxic air contaminants (N-nitrosamines, aldehydes, and other VOCs, including 1,3-butadiene) in a room-sized environmental chamber under conditions which simulate typical indoor settings;

2. Estimate the range and variability of the ETS emission factors among a subset of popular cigarette brands which have large market shares in California;

3. Determine emission factors for the same VOCs in sidestream smoke for comparison with the ETS measurements; and 
4. Investigate the effect of aging on the apparent emission factors of 1,3-butadiene and other VOCs in ETS.

Emission factors were determined for volatile $\mathrm{N}$-nitrosamines, aldehydes (formaldehyde, acetaldehyde, and acrolein), and for 20 additional VOCs including pyridine, pyrrole, and 3-vinylpyridine. The latter three compounds were investigated as possible tracers for the vapor-phase components of ETS. As part of this research, we also measured vapor-phase nicotine in ETS, total nicotine in SS, suspended particulate matter (PM-2.5 $\left.5_{\mathrm{EQ}}\right)^{*}$ in ETS, total condensed particulate matter in SS and $\mathrm{NO}_{\mathrm{x}}$ and CO in ETS. Nicotine was measured because it is commonly used as a tracer for estimating exposures to particles from ETS. Airborne particulate matter was measured so that its ratio to nicotine for various brands of cigarettes could be compared to those measured by other investigators and to provide an emission factor for estimating Californians' exposures to ETS particulate matter.

ETS emission factors were determined from concentration measurements made in a room-sized $\left(20-\mathrm{m}^{3}\right)$ environmental chamber with stainless steel walls and low background concentrations of the species of interest. The chamber was operated under static conditions, with a minimal air exchange rate of $0.029 \mathrm{~h}^{-1}$ for 4.5 hours for each experiment. Diluted sidestream smoke, emitted into the chamber and mixed, was used to simulate ETS. In each experiment, three cigarettes were smoked by machine using a standard smoking cycle of one puff per minute of $35-\mathrm{cm}^{3}$ volume and 2-sec duration. Sampling was initiated immediately after smoking was completed. The air in the chamber was mixed throughout the entire experiment, and a fluorescent light simulated indoor lighting conditions. For the determination of SS emission factors, an apparatus of $225-\mathrm{mL}$ volume, designed and used by Brunnemann and Hoffmann (1974), was utilized to sample freshly generated and minimally diluted sidestream smoke. The airflow rate through the apparatus was $1.5-\mathrm{L} \mathrm{min}^{-1}$.

Six commercial cigarettes were selected for testing: Benson and Hedges, filtered, mentholated; Camel, non-filtered; Marlboro, filtered; Marlboro, filtered, light; Salem, filtered, mentholated; and Winston, filtered. Kentucky reference cigarette $1 R 4 F$ (filtered), which is made to reflect current U.S. market shares of various cigarettes (except extra-light types), was also tested. The selected commercial brands represent $62.5 \%$ of the market share (population weighted) of cigarettes sold in California in 1990 and include filtered, non-filtered and mentholated cigarettes. The brands selected are also reasonably representative of the brands favored by various ethnic groups in California. Four different cigarette manufacturers are represented.

\footnotetext{
* Total airborne particulate matter was collected in the chamber. Based on other studies in our laboratory, $98 \%$ of the particles collected in the chamber would be less than $0.8 \mu \mathrm{m}$. Thus, the samples were equivalent to those which would have been measured with a PM- 2.5 sampler and are designated PM-2.5 $5_{\mathrm{EQ}}$.
} 
As part of this study, a method for sampling vapor-phase N-nitrosamines in cigarette smoke using a commercial sampling cartridge (Thermosorb/N) was developed and validated. The sample collection method is reliable and free of artifacts. Sample clean-up procedures for volatile N-nitrosamines were also developed and validated. The reproducibility and the precision of the method are enhanced over those for the traditional bubbler method.

\subsection{Determination of the ETS emission factors ( $\mu \mathrm{g} /$ cigarette) for selected toxic air contaminants in a room-sized environmental chamber under conditions which simulate typical indoor settings}

Table 1.1 summarizes the averages and standard deviations of the ETS emission factors determined for the six commercial brands of cigarettes and reference cigarette $1 \mathrm{R} 4 \mathrm{~F}$. The emission factors are presented in terms of $\mathrm{ng}$ of compound per $\mathrm{mg}$ of tobacco smoked and of $\mu \mathrm{g}$ per cigarette. The uncertainties for sampling and analysis are also shown in the table. These were estimated using the method of propagation of errors since multiple measurements of a single value were not made. The uncertainties for sampling and analysis ranged from about 6 to $25 \%$. These uncertainties were mostly associated with the analyses. Emission factors determined in duplicate chamber experiments were in excellent agreement and indicated that any variabilities in chamber operation did not noticeably add to the uncertainties in the measurements. The variabilities of duplicate SS measurements were also consistent with the estimated uncertainties.

The emission factors for the target compounds range from $0.1 \mu \mathrm{g} /$ cigarette for $\mathrm{N}$-nitrosopyrrolidine to as high as $2,150 \pm 477 \mu \mathrm{g} /$ cigarette for acetaldehyde. Butyl acetate, butyraldehyde, ethyl acetate, ethyl acrylate, 3-methyl-1-butanol, $\mathrm{N}$-nitrosodiethylamine and N-nitrosomorpholine were not detected in any of the ETS or SS samples. For these compounds, the emission factors were calculated using the minimum detection limits and were reported as less-than values. Sample sizes could not be increased to improve the limits of detection for the VOCs without overloading and contaminating the analytical instrument with the more abundant sample components. The "light" cigarette brands emitted slightly more volatile $\mathrm{N}$-nitrosamines than the regular cigarette brands. The emission factors for reference cigarette $1 \mathrm{R} 4 \mathrm{~F}$ generally fell within the range of the factors determined for the commercial cigarettes or were very close (Table 1.1). Although acrolein was collected and analyzed in all of the experiments, there was evidence of interferences in the analyses and/or chemical reactions during sampling. For these reasons, we do not recommend the use of the acrolein emission factors for exposure estimation.

Nicotine is a semi-volatile organic compound which is emitted is large amounts during cigarette combustion. An apparent emission factor is reported for nicotine in Table 1.1. This apparent emission factor is based on time-averaged concentrations measured in chamber air over the four-hour sampling 
Table 1.1. Summary of environmental tobacco smoke emission factors determined for six commercial cigarettes and Kentucky reference cigarette 1R4F.

\begin{tabular}{|c|c|c|c|c|c|}
\hline \multirow[t]{2}{*}{ Compound } & \multicolumn{2}{|c|}{$\begin{array}{c}\text { Emission Factor, } \\
\text { ng/mg }\end{array}$} & \multicolumn{2}{|c|}{$\begin{array}{c}\text { Emission Factor, } \\
\mu \mathrm{g} / \mathrm{cig} .\end{array}$} & \multirow[t]{2}{*}{$\begin{array}{l}\text { Uncertainty }^{\mathrm{b}} \\
\text { of Emission } \\
\text { Factors, \% } \\
\end{array}$} \\
\hline & $\begin{array}{l}\text { Average } \pm \\
\text { Std. Dev. }{ }^{2}\end{array}$ & 1R4F & $\begin{array}{l}\text { Average } \pm \\
\text { Std. Dev. }\end{array}$ & 1R4F & \\
\hline Acetaldehyde & $3,340 \pm 525$ & 3,430 & $2,150 \pm 477$ & 2,220 & 14 \\
\hline Acrolein $^{c .}$ & $(126 \pm 109)$ & $(120)$ & $(86 \pm 86)$ & (78) & 14 \\
\hline Acrylonitrile & $154 \pm 16$ & 185 & $99 \pm 18$ & 120 & 16 \\
\hline Benzene & $630 \pm 31$ & 653 & $406 \pm 71$ & 423 & 7 \\
\hline 1,3-Butadiene & $236 \pm 29$ & 276 & $152 \pm 27$ & 179 & 25 \\
\hline 2-Butanone (MEK) & $451 \pm 25$ & 585 & $291 \pm 56$ & 379 & 8 \\
\hline Butyl acetate $^{\mathrm{d} .}$ & $<4$ & $<4$ & $<3$ & $<3$ & 7 \\
\hline Butyraldehyde & $<29$ & $<29$ & $<18$ & $<18$ & 7 \\
\hline $\mathrm{m}, \mathrm{p}-\mathrm{Cresol}^{\mathrm{e}}$ & $128 \pm 27$ & 106 & $83 \pm 26$ & 68 & 14 \\
\hline o-Cresol ${ }^{e}$ & $55 \pm 11$ & 59 & $35 \pm 5$ & 38 & 15 \\
\hline Ethyl acetate $^{\mathrm{d} .}$ & $<6$ & $<6$ & $<4$ & $<4$ & 7 \\
\hline Ethyl acrylate ${ }^{\text {d. }}$ & $<5$ & $<5$ & $<3$ & $<3$ & 14 \\
\hline Ethylbenzene & $157 \pm 14$ & 178 & $130 \pm 10$ & 89 & 7 \\
\hline Formaldehyde & $2,040 \pm 414$ & 2,060 & $1,310 \pm 348$ & 1,330 & 10 \\
\hline 3-Methyl-1-butanol ${ }^{\mathrm{d}}$ & $<23$ & $<23$ & $<14$ & $<14$ & 17 \\
\hline Nicotine & $1,410 \pm 260$ & 1,540 & $919 \pm 240$ & 993 & 9 \\
\hline N-Nitrosodiethylamine ${ }^{d}$ & $<0.033$ & $<0.033$ & $<0.020$ & $<0.020$ & 13 \\
\hline N-Nitrosodimethylamine & $0.88 \pm 0.11$ & 0.69 & $0.57 \pm 0.12$ & 0.44 & 13 \\
\hline N-Nitrosomorpholine $\mathrm{e}^{\mathrm{d} .}$ & $<0.033$ & $<0.033$ & $<0.020$ & $<0.020$ & 13 \\
\hline N-Nitrosopyrrolidine & $0.16 \pm 0.02$ & 0.15 & $0.10 \pm 0.02$ & 0.10 & 13 \\
\hline Phenol $^{\text {e. }}$ & $438 \pm 76$ & 368 & $281 \pm 61$ & 238 & 9 \\
\hline Pyridine & $663 \pm 126$ & 989 & $428 \pm 122$ & 641 & 18 \\
\hline Pymole & $626 \pm 94$ & 816 & $402 \pm 90$ & 529 & 16 \\
\hline Styrene & $229 \pm 16$ & 250 & $147 \pm 24$ & 162 & 15 \\
\hline Toluene & $1,020 \pm 78$ & 1,130 & $656 \pm 107$ & 732 & 6 \\
\hline 3-Vinylpyridine ${ }^{e}$ & $1,020 \pm 149$ & 1,054 & $662 \pm 155$ & 683 & 10 \\
\hline m,p-Xylene & $467 \pm 40$ & 504 & $299 \pm 52$ & 327 & 7 \\
\hline o-Xylene & $104 \pm 13$ & 115 & $67 \pm 16$ & 75 & 6 \\
\hline PM-2.5 & $12,400 \pm 1,300$ & 11,900 & $8,100 \pm 2,000$ & 7,700 & 2 \\
\hline
\end{tabular}
a. Average \pm Standard Deviation for six commercial cigarettes.
b. Estimated by propagation of errors method for $\mu \mathrm{g} / \mathrm{cig}$ values.
c. Use of the acrolein emission factors for exposure modeling is not recommended.
d. Less-than values are lower limits of detection.
e. Emission factors are corrected for deposition losses to chamber surfaces. 
period. However, based on our measurements of total nicotine in SS, approximately $80 \%$ rapidly deposited to the surfaces of the chamber or was otherwise lost. At present, the theory of deposition losses (and reemission) for such compounds is not sufficiently advanced that we can estimate indoor air concentrations with confidence. Nevertheless, the apparent emission factor is in good agreement with the nicotine emission factor measured by Leaderer and Hammond (1991) in an environmental chamber. Despite the expected variability in nicotine deposition and re-emission from different surfaces, the ratio of airborne ETS particulate matter to ETS nicotine for our chamber experiments is also in good agreement with ratios reported for other chamber experiments (Hammond, et al., 1987; Lofroth, et al., 1989; Leaderer and Hammond, 1991) and for measurements made in U.S. residences (Leaderer and Hammond, 1991).

The average emission factor for PM-2.5 $5_{\mathrm{EQ}}$, corrected for surface deposition, was $8.1 \pm 2.0 \mathrm{mg} / \mathrm{cig}$. The PM-2.5 $5_{\mathrm{EQ}}$ emission factors were 29 and $44 \%$ lower than those respectively reported by Lofroth, et al. (1989) and by Leaderer and Hammond (1991). There is evidence that suggests that the reason for this difference is attributable to the smoking protocols (Benner, et al., 1989). In the two earlier chamber studies, cigarettes were continuously smoked by human subjects during the sampling period and a mixture of freshly generated and aged ETS was sampled. In our experiments, samples were collected for a fourhour period after completion of smoking. Thus, there was sufficient time for some volatilization of particle mass to the vapor phase and a consequent lowering of the PM-2.5 $5_{\mathrm{EQ}}$ emission factor. In addition, our study did not include a contribution from exhaled mainstream smoke.

Three nitrogen-containing VOCs, pyridine, pyrrole, and 3-vinylpyridine, were investigated as potential tracers for vapor-phase compounds from ETS. Criteria for an ETS tracer include (NRC, 1986): 1) uniqueness to tobacco smoke; 2) detectability at low smoking rates; 3) similar emission rates across different tobacco products; and 4) consistent proportions to other ETS compounds for different environments and tobacco products. Based on emissions data reported in the literature and measurements of VOCs in indoor environments, these three VOCs appear to be unique to tobacco smoke in indoor air. All three compounds can be easily detected at low smoking rates, although they are not as abundant in ETS as nicotine. The coefficients of variation for the pyridine, pyrrole and 3-vinylpyridine emission factors $(\mu \mathrm{g} / \mathrm{cig})$ among the six commercial brands of cigarettes were 29,22 , and $23 \%$ respectively. The ratios of the highest to lowest emission factors for these compounds were about 2 or less. Thus, all three compounds meet the third criterion reasonably well. A tracer must also exhibit indoor behavior similar to that of the vapor-phase compounds it traces. Based on our measurements, only pyridine and pyrrole meet this criterion. The 3-vinylpyridine was found to deposit onto surfaces in the chamber over the period of the experiments. Thus, mass-balance estimates of the contributions of ETS to the indoor concentrations of vapor-phase VOCs which have multiple indoor sources (e.g., benzene, styrene and toluene) should be based on their ratios to pyridine and/or pyrrole as the tracer compounds. Further work in buildings to more fully evaluate 
the use of pyridine and pyrrole as tracers of VOCs from ETS is currently in progress in our laboratory for another project.

\subsection{Estimation of the range and variability of the ETS emission factors among a subset of popular cigarettes brands which have large market shares in California}

The variability in the ETS emission factors among brands of cigarettes was quite small. The emission factors expressed as mass per mg of tobacco smoked typically varied by $15 \%$ or less, and these variations were similar to the uncertainties due to sampling and analysis. The ETS emission factors as mass per cigarette showed slightly greater variabilities among cigarette brands of 16 to $29 \%$ due to differences in the cigarette lengths and tobacco densities. The emission factors for 3-vinylpyridine, phenol, o-cresol and $\mathrm{m}, \mathrm{p}$-cresol were corrected for deposition losses to the interior surfaces of the stainless-steel chamber. The variability among brands was generally somewhat greater for these four compounds. The ratios of the highest to the lowest emission factors $(\mu \mathrm{g} / \mathrm{cig})$ among cigarette brands was 1.5 to 1.6 for most of the VOCs and ranged up to 2.0 for pyridine. Mentholation of the cigarettes did not seem to affect the ETS emissions of the target VOCs. The ETS emissions of light and regular cigarettes were also not significantly different for most of the VOCs. Only the volatile $\mathrm{N}$-nitrosamines showed significant differences.

\subsection{Determination of emission factors for the same VOCs in sidestream smoke for comparison with the ETS measurements}

A summary of the SS emission factors is presented in Table 1.2. Emission factors for the undiluted SS were generally significantly lower than those for simulated ETS (diluted and aged SS). There is no internationally recognized procedure for sidestream smoke production. SS emission factors can vary significantly depending on the apparatus and procedures that are used. Important parameters are air dilution, air flow near the burning cigarette cone, and the size of the chamber. These can vary significantly between a typical SS apparatus and an environmental chamber. For example, since the SS apparatus has a much higher surface-to-volume ratio than does the environmental chamber, there is a much greater potential for losses of compounds to surfaces. There is also a greater potential for losses of reactive compounds due to chemical reactions in the SS apparatus because of the higher concentration of cigarette smoke.

The lowest SS emission factors relative to ETS factors occurred for the most reactive VOCs, formaldehyde and 1,3-butadiene, and for the VOCs with the lowest vapor pressures, phenol, cresols, and 3vinylpyridine. The high concentrations of smoke, water vapor and ammonia in the SS chamber may have promoted chemical reactions of formaldehyde. 1,3-Butadiene may have also been lost due to chemical reactions. The less-volatile VOCs may have been partially lost through condensation on the surface of the SS chamber and on the filter used to protect the sampling device. 
It may be possible to design a larger bench-scale SS apparatus that would produce emission factors more similar to those measured in the room-sized chamber. Such a device should have air dilution and air flow patterns around the burning cigarette that more closely simulate smoking environments. However, the effort required to develop and validate a new apparatus may not be cost effective since most of the costs of determining ETS emission factors in a large chamber are associated with sampling and the chemical analyses.

The ETS emission factors for vapor-phase nicotine were significantly lower than the SS emission factors for total nicotine which included the condensates from the SS apparatus. Nicotine is a semi-volatile compound with a very low vapor pressure at room conditions. Significant amounts of this compound were presumably lost by deposition onto the interior surfaces of the environmental chamber.

Particulate matter emission factors were also higher in the SS apparatus than in the environmental chamber. This was probably due to artifacts from condensation and sorption on the sampling filters as the result of the very high concentrations of compounds in the gas stream of the SS apparatus.

\subsection{Investigation of the effect of aging on the apparent emission factors of 1,3-butadiene and other VOCs in ETS}

Aldehydes were measured over the first and last 100 minutes of the ETS chamber experiments to detect any losses that might occur due to chemical reactions. The average ratios of the emission factors for the last and first samples were near one for formaldehyde, acetaldehyde and acrolein indicating that chemical reactions involving these compounds did not occur or were minimal over the four-hour sampling period. 
Table 1.2. Comparison of ETS and SS emission factors determined for six commercial brands of cigarettes, $\mu \mathrm{g} /$ cigarette.

\begin{tabular}{|c|c|c|c|}
\hline Compound & $\begin{array}{l}\text { ETS Emission Factors } \\
\text { Average } \pm \text { Std. Dev. }^{2}\end{array}$ & $\begin{array}{c}\text { SS Emission Factors } \\
\text { Average } \pm \text { Std. Dev. }^{2}\end{array}$ & $\begin{array}{c}\text { Factors Significantly } \\
\text { Different }(p<0.01)^{b}\end{array}$ \\
\hline Acetaldehyde & $2,150 \pm 477$ & $1,660 \pm 279$ & Yes; ETS >SS \\
\hline Acrolein $^{c}$ & $(86 \pm 86)$ & $(38 \pm 12)$ & No \\
\hline Acrylonitrile & $99 \pm 18$ & $69 \pm 18$ & Yes; ETS $>$ SS \\
\hline Benzene & $406 \pm 71$ & $248 \pm 70$ & Yes; ETS $>$ SS \\
\hline 1,3-Butadiene & $152 \pm 27$ & $<58$ & Yes; ETS>SS \\
\hline 2-Butanone (MEK) & $291 \pm 56$ & $197 \pm 52$ & Yes; ETS $>$ SS \\
\hline Butyl acetate ${ }^{\mathrm{d}}$ & $<3$ & $<2$ & -- \\
\hline Butyraldehyde ${ }^{d .}$ & $<18$ & $<14$ & -- \\
\hline $\mathrm{m}, \mathrm{p}-\mathrm{Cresol}^{\mathrm{e}}$ & $83 \pm 26$ & $<18$ & Yes; ETS>SS \\
\hline o-Cresol ${ }^{e}$ & $35 \pm 5$ & $<18$ & Yes; ETS $>$ SS \\
\hline Ethyl acetate ${ }^{\mathrm{d}}$ & $<4$ & $<3$ & -- \\
\hline Ethyl acrylate $e^{d .}$ & $<3$ & $<3$ & -- \\
\hline Ethylbenzene & $130 \pm 10$ & $84 \pm 17$ & Yes; ETS >SS \\
\hline Formaldehyde & $1,310 \pm 348$ & $28 \pm 7$ & Yes; ETS $>$ SS \\
\hline 3-Methyl-1-butanol ${ }^{\mathrm{d} .}$ & $<14$ & $<11$ & --- \\
\hline Nicotine & $919 \pm 240$ & $5,060 \pm 760$ & Yes; SS>ETS \\
\hline N-Nitrosodiethylamine ${ }^{\mathrm{d}}$ & $<0.020$ & $<0.002$ & - \\
\hline $\mathrm{N}$-Nitrosodimethylamine & $0.57 \pm 0.12$ & $0.28 \pm 0.06$ & Yes; ETS $>$ SS \\
\hline $\mathrm{N}$-Nitrosomorpholine ${ }^{\mathrm{d} .}$ & $<0.020$ & $<0.002$ & -- \\
\hline N-Nitrosopyrrolidine & $0.10 \pm 0.02$ & $0.14 \pm 0.02$ & Yes; SS>ETS \\
\hline Phenol $^{\text {e. }}$ & $281 \pm 61$ & $<47$ & Yes; ETS $>$ SS \\
\hline Pyridine & $428 \pm 122$ & $394 \pm 80$ & No \\
\hline Pyrrole & $402 \pm 90$ & $411 \pm 87$ & No \\
\hline Styrene & $147 \pm 24$ & $102 \pm 22$ & Yes; ETS $>$ SS \\
\hline Toluene & $656 \pm 107$ & $508 \pm 86$ & Yes; ETS $>$ SS \\
\hline 3-Vinylpyridine $e^{e .}$ & $662 \pm 155$ & $298 \pm 74$ & Yes; ETS $>S S$ \\
\hline $\mathrm{m}, \mathrm{p}$-Xylene & $299 \pm 52$ & $224 \pm 56$ & Yes; ETS $>$ SS \\
\hline o-Xylene & $67 \pm 16$ & $44 \pm 10$ & Yes; ETS $>$ SS \\
\hline PM-2.5 & $8,100 \pm 2,000$ & $30,000 \pm 5,700$ & Yes; SS>ETS \\
\hline
\end{tabular}

\footnotetext{
a. Average \pm Standard Deviation for six commercial cigarettes.

b. Paired t-test.

c. Use of the acrolein emission factors for exposure modeling is not recommended.

d. Less-than values are lower limits of detection.

e. ETS emission factors are corrected for deposition losses to chamber surfaces.
} 
For the other VOCs, chamber concentrations were measured over time by collecting four successive one-hour samples. The concentrations and calculated emission factors for 1,3-butadiene showed some evidence of increases with time in several experiments. However, since these increases were not consistent and there was a relatively high amount of uncertainty in the measurement of this compound, it could not be concluded that concentrations of 1,3-butadiene changed significantly with time. The chamber concentrations of four of the VOCs, 3-vinylpyridine, phenol, m,p-cresol and o-cresol, consistently decreased with time at rates that were greater than that predicted by the small ventilation rate for the chamber. These compounds were the least volatile of the target VOCs. The additional removal was undoubtedly due to deposition of the compounds onto the interior surfaces of the chamber. Removal rates due to deposition losses were calculated for the four VOCs. These rates ranged from 0.09 to $0.27 \mathrm{~h}^{-1}$ and were related to the vapor pressures of the compounds. As discussed above, the loss of nicotine, a semivolatile compound, to chamber surfaces was substantially greater. Loss rates of these compounds in buildings with typical surface materials would be expected to be higher than those measured in the stainlesssteel chamber. Therefore, the ETS emission factors for 3-vinylpyridine, phenol and cresols will over estimate population exposures to these compounds when they are used in mass-balance models unless proper account is taken of these losses.

\subsection{Use of emission factors for modeling exposures of the California population to selected VOCs in ETS}

As noted above, SS measurements are predicted to be highly dependent upon the design and operation of the SS apparatus. The environmental chamber, on the other hand, much more closely simulates real smoking environments. For almost all of the target VOCs, the ETS emission factors were significantly higher than the corresponding SS emission factors. This was presumably due to differences in the combustion conditions, as well as wall loses and possibly chemical reactions in the SS apparatus. Therefore, the ETS emission factors measured in the environmental chamber, rather than the SS emission factors, are recommended for use in models to estimate population exposures.

The ETS emission factors presented in Table 1.1 are representative of the emissions from the major brands of cigarettes smoked by Californians. The variabilities in emission factors ( $\mu \mathrm{g} / \mathrm{cig}$ ) among the six brands expressed as coefficients of variation were 16 to $29 \%$. Mentholation, or whether a cigarette was classified as light or regular, did not significantly affect the emissions of the target compounds. The differences among brands were, however, related to the lengths and tobacco densities of the cigarettes as evidenced by the lower variabilities when the results were expressed as mass of compound emitted per milligram of tobacco consumed. Because of the relative uniformity of the emissions and the relationship with the amount of tobacco consumed, emissions from other cigarettes can probably be predicted with a high degree of confidence using the ETS data from this study. 
The measurements of the concentrations of the target compounds were generally of high quality. This lends confidence to model predictions that are made using these data as inputs. An improved sampling method for volatile $\mathrm{N}$-nitrosamines using a solid sorbent cartridges was developed and validated as part of this study. The overall method is highly sensitive, has good precision, and produced results that were comparable to literature values obtained with less convenient methods. The one exception is acrolein, for which there were analytical problems. It is suspected that there were substantial losses of acrolein in the sampling system which lead to low ETS and SS emission factors relative to some values that have been reported in the literature using other methods. Therefore, we do not recommend the use of the acrolein emission factors from this study for exposure modeling.

Aging of ETS was determined not to be a significant factor for the compounds included in this study. They were chemically stable and were neither created or consumed during the four-hour experimental period. There were, however, deposition losses of the less volatile compounds to chamber surfaces. These losses were presumably severe for nicotine as evidenced by the five-fold difference between total nicotine produced in the SS experiments and vapor-phase nicotine measured in the environmental chamber. The emission factors for 3-vinylpyridine, phenol and cresols were corrected for deposition losses in the chamber. At present, we have no means of reliably estimating such losses to complex surfaces in buildings. It is also likely that these compounds are re-emitted from surfaces under some conditions. Exposure estimates for the lower volatility compounds that are based solely on the corrected ETS emission factors are likely to be overestimates.

Two potential tracers for VOCs from ETS were identified and were shown to meet most of the criteria for such use. These are the nitrogen containing compounds pyridine and pyrrole. Of these two, analytical sensitivity was betisr for pyrrole. By using such a volatile tobacco-specific tracer, it should be possible to determine the contributions of ETS to airborne concentrations in buildings of compounds, such as the aromatic hydrocarbons, which have other indoor sources besides ETS. Additional work will be required to determine the behavior of pyridine and pyrrole in buildings. 


\subsection{RECOMMENDATIONS}

\subsection{Surface deposition and re-emission rates of VOCs}

Several of the target compounds included in this study showed evidence of deposition losses to the interior surfaces of the environmental chamber. Loss rate constants were determined for these compounds in the stainless-steel chamber. However, these constants cannot be applied to other environments, such as buildings, because surface materials, surface-to-volume ratios, airflow characteristics near surfaces, and bulk air concentrations will be different. Sorbed compounds will also desorb when their bulk air concentrations decrease. Therefore, it is not possible to make accurate estimates of the indoor concentrations of these compounds using the emission rate data obtained in a stainless-steel chamber. An empirical approach using different surfaces and building parameters could be attempted to generate loss rate constants for a variety of settings. A more general theoretical framework for deposition and re-emission of VOCs in indoor environments could also be developed through additional research. These studies would provide the data that are needed to accurately predict indoor concentrations of the less volatile compounds in ETS.

\subsection{Improved sampling method for acrolein}

The measured emission rates of acrolein in both SS and ETS were unexpectedly low. It is suspected that there were substantial losses of this compound in the acid impregnated cartridges used to collect the samples. The method employing these cartridges is widely used for sampling aldehydes in indoor and outdoor air. However, it does not appear to have been adequately validated for all of the target compounds. A validation study should be conducted using a known vapor source of acrolein at low partper-billion concentrations as the standard. An alternate sampling method for acrolein may need to be developed.

\subsection{Tobacco-specific tracers for VOCs in ETS}

This study has provided data which suggest that pyridine or pyrrole may be useful as tracers for the more-volatile components of ETS. Considerable additional work is needed to evaluate these potential tracers. The issue of analytical sensitivity and precision needs further investigation as the emission rates for these compounds are not elevated above the emission rates of the compounds of interest, such as benzene. It should be confirmed that ETS is the primary source of these compounds in indoor environments. The ratios of the tracers to the target compounds should be carefully evaluated in a chamber study employing different surface materials and environmental conditions. Finally, the usefulness of these compounds as tracers needs to be demonstrated in real buildings. Some of this evaluation is currently being conducted at LBL with other sources of funding. 


\subsection{Loss mechanisms for nicotine}

Because it is unique to tobacco smoke and is present at relatively high abundance, nicotine is commonly used as a tracer for exposure to particles in ETS. However, its behavior in indoor environments is not completely understood. In the environmental chamber, the airborne nicotine concentration was a factor of four or five lower than that predicted from the total amount nicotine generated in the SS experiments. Much of the nicotine may be lost by deposition to surfaces and re-emission may occur over relatively long time periods. There may also be losses due to chemical reactions. A mass balance study is needed to identify all of the sinks for nicotine. The deposition and re-emission of nicotine from surfaces should also be studied in more detail. An improved understanding of the behavior of nicotine should improve its utility as tracer for ETS. Some additional study of nicotine is currently being conducted at LBL with other sources of funding.

\subsection{Measurement of emission factors in buildings}

The usefulness of ETS emission factors obtained by this and other chamber studies should be validated by conducting similar measurements in several indoor environments with real smokers and real surface materials and furnishings. All of the same parameters would need to be controlled and measured. These parameters include smoking rates, ventilation rates, and background and ventilation air concentrations of the target compounds. Measured ETS emission factors could then be compared with those predicted from the chamber experiments. 


\subsection{STATEMENT OF PROBLEM}

\subsection{Background}

The California Air Resources Board (CARB) is required to consider indoor exposures in assessing risks to public health posed by compounds classified as or being reviewed for classification as toxic air contaminants. Consequently, a goal of the Indoor Air Quality/Personal Exposure Assessment Program (Indoor Program) of the CARB is to obtain data that are applicable to California for such toxic compounds. To achieve these goals, the Indoor Program sponsors a number of interrelated research activities, the objectives of which are to: 1) obtain data regarding health effects; 2) develop monitoring methods if suitable methods are unavailable; 3) obtain indoor concentration and exposure pattern data; 4) identify indoor sources and obtain source emissions data; 5) determine activity patterns for Californians; and, 6) develop and validate a total exposure model which can be used to perform risk assessments.

For some of the compounds, the existing data on indoor concentrations and personal exposures are adequate with respect to the CARB's goals. However, for many, the sources of indoor exposure have not been fully identified, and no, or only limited, indoor concentration and personal exposure data are available. To obtain more information, the CARB has sponsored field surveys to measure indoor concentrations and exposures for a variety of compounds and has initiated a research effort to identify indoor sources and measure source emission rates of selected candidate compounds.

As part of these efforts, Lawrence Berkeley Laboratory (LBL) conducted a literature study to evaluate published data on indoor concentrations and personal exposures for selected volatile organic compounds (VOCs) that were being reviewed as candidate toxic air contaminants (Hodgson and Wooley, 1991). This study identified environmental tobacco smoke (ETS) as an important source of a number of the toxic air contaminant VOCs in indoor air, including 1,3-butadiene, acetaldehyde, acrolein, N-nitrosamines, cresols, phenols, and hydrazine. Recent research indicates that $62 \%$ of adults and adolescents in California are exposed to ETS for an average of about 5 hours per day (Wiley, et al., 1991). Thus, exposures to these VOCs from this source alone are widespread in California. Although ETS is likely to be the major source of exposure for many of the toxic air contaminants, quantitative data in support of this hypothesis are often lacking or weak (Daisey, et al., 1991).

\subsection{Environmental Tobacco Smoke}

Tobacco smoke is a highly complex mixture of thousands of volatile, semi-volatile and particulate organic and inorganic compounds of which 300-400 have been identified in sidestream smoke (NRC, 1986; Hoffmann and Wynder, 1986). The polycyclic aromatic hydrocarbons (PAH) and the tobacco-specific Nnitrosamines present in tobacco smoke are suspected to play a significant role in its overall carcinogenic properties (Hoffmann and Wynder, 1986). For example, the $\mathrm{N}$-nitrosamine compounds are among the most 
potent chemical carcinogens in experimental animals (IARC, 1978). The U.S. EPA has assigned a unit risk value for $\mathrm{N}$-nitrosodimethylamine (NDMA) of $1.4 \times 10^{-2}$ per $\mu \mathrm{g} \mathrm{m}^{-3}$. The concentration at which the excess cancer risk for NDMA is estimated to be equal to one in a million $\left(10^{-6}\right)$ is $7 \times 10^{-5} \mu \mathrm{g} \mathrm{m}^{-3}$ or $2.3 \mathrm{x}$ $10^{-5}$ ppbv (CARB, 1989). Other carcinogens, co-carcinogens, and cancer promoting agents are also present in tobacco smoke and probably contribute to its carcinogenicity.

Environmental tobacco smoke (ETS) is the smoke to which non-smokers are exposed when they are in an indoor environment with smokers. It is composed largely of sidestream tobacco smoke (SS), the smoke emitted by the smoldering end of a cigarette between puffs, with minor contributions from exhaled mainstream smoke (the smoke from the cigarette which is directly inhaled by the smoker) and any smoke that escapes from the burning part of the tobacco during puff-drawing by the smoker. ETS differs from SS in that it is highly diluted and dispersed within a room and it undergoes aging. There is a growing body of evidence which indicates that exposure to ETS increases the risk of lung cancer, although exposures and risks are lower than for mainstream smoke (U.S. EPA, 1992).

Sidestream smoke, the main component of ETS, differs somewhat from mainstream smoke in its chemical composition. For example, the emissions of benzene, acrolein, $\mathrm{N}$-nitrosamines, aromatic amines and polycyclic aromatic hydrocarbons are higher in freshly diluted sidestream smoke than in mainstream smoke by factors ranging from 2 to 100 (NRC, 1986). In addition, the $\mathrm{pH}$ of sidestream smoke ranges from 6.7 to 7.5 while that of mainstream smoke ranges from about 6.0 to 6.5 . One consequence of the higher $\mathrm{pH}$ of sidestream smoke is that it contains a greater proportion of unprotonated nicotine and more nicotine in the vapor than in the particulate phase (Hoffmann and Wynder, 1986). These differences are due in part to differences in combustion temperatures and fuel-to-oxygen ratios for the generation of sidestream and mainstream smokè.

\subsection{Limitations of Available Data on ETS}

Emission factors for many VOCs have been reported for sidestream smoke freshly collected from smoldering cigarettes. The estimates for individual compounds in SS generally range over a factor of about two to five (NRC, 1986), but data on variability are fairly limited. It should be noted that the range of emission factors for SS seems to be smaller than for mainstream smoke. Emission factors have not generally been measured for ETS. Furthermore, there have been only a few limited comparisons between emission factors measured for SS and ETS. If the ETS and SS emission factors could be shown to be similar, then those available for SS could be used to model exposures to many VOCs in ETS with more confidence.

Another problem with the existing data base on SS emission factors is that the specific brands of cigarettes smoked are not identified. Thus, it is difficult to use these data to represent the brands of 
cigarettes which are being smoked in California without making the assumption that emission factors do not differ significantly among different brands and types of cigarettes. In addition, emission factors for SS or ETS reported in the older literature may differ from emission factors for current cigarettes because of changes in cigarette manufacturing processes to reduce tar in mainstream smoke, e.g., more use of reconstituted tobacco.

There have been a few measurements of the concentrations of some of the candidate VOCs in ETS in rooms with smokers but, in these experiments, neither the air exchange rates for the room nor the background (non-ETS) concentrations of the VOCs were reported. Thus, emission factors cannot be inferred from these uncontrolled experiments.

There is also the issue of whether chemical reactions of ETS, which may occur over time (i.e., "aging"), have a significant impact on measured emission factors. At present, we have almost no quantitative information on reactivity losses over time. If the losses are large, then the emission factors measured immediately after a cigarette is smoked would significantly overestimate exposures. This information cannot be inferred from measurements of sidestream smoke since this is always collected directly and immediately from the smoldering cigarette. There is some limited evidence that some reactions do occur as ETS ages. For example, nitrogen oxide (NO) has been shown to gradually oxidize to nitrogen dioxide $\left(\mathrm{NO}_{2}\right)$ as ETS ages (Villains and Lephardt, 1975). Schmeltz and Hoffmann (1977) have suggested that amines in ETS may be converted to N-nitrosamines with time but have not done experiments to test this hypothesis. For compounds such as 1,3-butadiene, which is quite reactive chemically, it is reasonable to expect some loss over time. For other less reactive compounds, such as benzene, losses from chemical reactions should be small.

There are many VOCs which have been identified in cigarette smoke (Ogden, 1988) for which we lack emission factors. Since many of these compounds can be quantitatively collected on the multisorbent sampler which is routinely used in our laboratory for the collection and analysis of a broad spectrum of VOCs in indoor air, we were able to cost-effectively measure a number of VOCs without additional method development.

Table 3.1 presents a list of VOCs and aldehydes identified in tobacco smoke which are on CARB's list of Toxic Air Contaminants (CARB, 1993). Methods for sampling and analyzing these compounds in air have been developed and validated and it was possible to determine emission factors for these compounds with only minor modifications in the methods. 
Table 3.1. Some VOCs and aldehydes reported in cigarette smoke ${ }^{2 \cdot}$ which are on CARB's list of toxic air contaminants.

\begin{tabular}{|c|c|c|c|}
\hline Compound & $\begin{array}{l}\text { CARB Toxic Air } \\
\text { Contaminant Group }\end{array}$ & VOCs $s^{b}$ & Aldehydes $^{c}$ \\
\hline Acetaldehyde & $\mathrm{I}$ & & $\bar{X}$ \\
\hline Acrolein & $I$ & & $\mathrm{X}$ \\
\hline Acrylonitrile & $\bar{I}$ & $\mathrm{X}$ & \\
\hline Benzene & $\bar{I}$ & $\mathrm{X}$ & \\
\hline 1,3-Butadiene & $I$ & $\bar{x}$ & \\
\hline n-Butyl acetate & & $\mathrm{X}$ & \\
\hline \multicolumn{4}{|l|}{ 2-Butanone } \\
\hline n-Butyraldehyde & & $\mathrm{X}$ & $\mathrm{X}$ \\
\hline Cresols ( 3 isomers) & $I$ & $\bar{X}$ & \\
\hline Ethyl acetate & & $\mathrm{X}$ & \\
\hline Ethyl acrylate & I & d. & \\
\hline Ethylbenzene & $I$ & $\mathrm{X}$ & \\
\hline Formaldehyde & $\bar{I}$ & & $\mathrm{X}$ \\
\hline 3-Methyl-1-butanol & & $\mathrm{X}$ & \\
\hline Phenol & $I$ & $\mathrm{X}$ & \\
\hline Styrene & I & $\bar{X}$ & \\
\hline Toluene & I & $\bar{X}$ & \\
\hline Xylenes ( 3 isomers) & $\bar{I}$ & $\mathrm{X}$ & \\
\hline
\end{tabular}

a. Ogden, 1988; NRC, 1986.

b. VOCs which can be sampled with a multisorbent sampler and analyzed by GC/MS

c. Volatile aldehydes which can be sampled on a silica cartridge with DNPH and analyzed by HPLC.

d. Ethyl acrylate was not reported in Ogden 1986, or NRC, 1988, but may be present since methyl acrylate has been reported.

Vapor-phase nicotine is frequently used as an indicator of exposure to particles from ETS. Field and laboratory measurements by Leaderer and Hammond (1991) have shown that vapor-phase nicotine is a suitable marker of exposures to respirable particulate matter (RSP, $D_{50}=3.5 \mu \mathrm{m}$ ) from ETS. However, because there have been so few simultaneous measurements of VOCs and nicotine in ETS, it is not clear how useful vapor-phase nicotine is as a measure of exposure to these vapor-phase components of ETS. If such data were available, then the measured ratios of the VOCs to nicotine could be used with field measurements of nicotine to estimate exposures to many toxic VOCs. 
In summary, there are some existing data for sidestream smoke that can be used to provide firstorder estimates (probably good to within a factor of about five) of exposures to some candidate VOCs from ETS. For some of the candidate VOCs, we lack quantitative measurements even in SS. We also lack information on how well ETS emission factors agree with the available SS values. In addition, we do not have very good data on the variability of emission factors across brands of cigarettes that are currently smoked in California. Existing indoor measurements cannot be used for modeling the exposures of the California population to toxic air contaminant VOCs from ETS because there is no information on background levels of the compounds of interest or on the air exchange rates for these spaces. Therefore, there is a need to measure ETS emission factors that can be used with confidence in appropriate models to estimate exposures of the California population to toxic air contaminants from this source. 


\subsection{OBJECTIVES}

The overall goal of the research reported here was to provide emission factors for selected candidate VOCs in ETS which can be used in models to estimate exposures of the California population. The specific objectives were to:

1. Determine the ETS emission factors ( $\mu \mathrm{g} /$ cigarette) for selected toxic air contaminants ( $N$-nitrosamines, aldehydes, and other VOCs, including 1,3-butadiene) in a room-sized environmental chamber under conditions which simulate typical indoor settings;

2. Estimate the range and variability of the ETS emission factors among a subset of popular cigarette brands which have large market shares in California;

3. Determine emission factors for the same VOCs in sidestream smoke for comparison with the ETS measurements; and

4. Investigate the effect of aging on the apparent emission factors of 1,3-butadiene and other VOCs in ETS.

Emission factors were determined for selected vapor-phase $\mathrm{N}$-nitrosamines and for the aldehydes (formaldehyde, acetaldehyde, and acrolein) and VOCs in Table 3.1. In addition, pyridine, pyrrole, and 3vinylpyridine were investigated as possible tracers for the vapor-phase components of ETS. As part of this research, we also measured vapor-phase nicotine in ETS and total nicotine in SS, suspended particulate matter in ETS and SS, and $\mathrm{NO}_{\mathrm{x}}$ and CO in ETS. Nicotine was measured because it is commonly used as a tracer for estimating exposures to particles from ETS. Airborne particulate matter was measured so that its ratio to nicotine for various brands of cigarettes could be compared to those measured by other investigators and also to provide a means of estimating exposures to ETS particulate matter from published measurements of vapor-phase nicotine. Airborne particulate matter (PM-10) is also one of the outdoor pollutants for which there are California and Federal standards. Concentrations of $\mathrm{NO}_{\mathrm{x}}$ were measured as a possible indicator of chemical reactions over time and $\mathrm{CO}$ was measured because it is not chemically reactive and would provide a means of checking that there was no unexpected air leakage in the environmental chamber during each experiment. 


\subsection{STUDY DESIGN}

\subsection{Overall Approach}

The overall approach in this study was to measure both ETS and SS emission factors for selected brands of commercially available cigarettes which represent a substantial share of the California market. Cigarettes were smoked by machine using standard protocols for both the ETS and SS measurements. The SS apparatus, originally designed by Neurath and Emke (1964) and modified by Brunnemann and Hoffmann (1974), was used to measure SS emission factors. ETS emission factors were determined using diluted SS in a room-sized environmental chamber. There were two reasons for using diluted SS to simulate ETS. Firstly, the chemical differences between the two are not expected to be large since SS constitutes the major portion of the ETS. Baker and Proctor (1990), for example, have reported that exhaled mainstream smoke constitutes only about $13 \%$ of the $\mathrm{CO}, 9 \%$ of the nicotine and $15 \%$ of the particles in ETS. Secondly, the alternative choice of using smokers in the chamber to generate ETS would require approval by the Human Use Committee and would be more costly since the smokers would have to be paid. Smoking would also be less controlled than with a smoking machine.

\subsection{Cigarettes}

\subsubsection{Selection of brands}

In consultation with CARB staff, six commercial cigarettes were selected for testing. This selection was based primarily on the market shares of major brands of cigarettes smoked in California and included high and low tar cigarettes. Kentucky reference cigarette $1 R 4 F$, which is made to reflect current U.S. market shares of various cigarettes (except extra-light types), was also tested.

Table 5.1 summarizes the market shares of the major brands of cigarettes purchased by smokers in California based on the 1990 California Tobacco Survey conducted by Westat for a University of California-San Diego project supported by the California Department of Health Services (DHS) (Pierce, et al., 1991; 1992). The survey included a question on cigarette brand preference. Specifically, the question asked was: "What brand do you usually buy?" The question was asked of current smokers. Current smokers were defined as respondents who have smoked at least 100 cigarettes in their lifetimes and who report current usage. The survey included almost 8000 current adult smokers who cited over 50 preferred cigarette brands. The data in Table 5.1 were generated using weighted frequencies from a data tape provided by the DHS Tobacco Control Section (H. Johnson, CARB, personal communication).

For each brand, there are a number of available types of cigarettes (e.g., filtered, non-filtered, mentholated, light, etc.). Information on the various types of cigarettes for each brand, was obtained from a U.S. Federal Trade Commission Report (FTC, 1992) which reports on the tar, nicotine and carbon 
monoxide content of the mainstream smoke of domestic cigarettes. In the FTC report, cigarette brands are sub-categorized by filter/non-filter, length of cigarette $[70 \mathrm{~mm}, 80-85 \mathrm{~mm}$ (King), and 100-120 mm], hard/soft pack, flavored/non-flavored; and light/ultra-light. For the purpose of selecting cigarettes for this study, the FTC categories were compressed. The packaging categories, hard and soft packs, were eliminated since they do not affect emissions. The length of the cigarette was also disregarded in the brand selection process since the tobacco weight and the length of each cigarette type was to be measured prior to testing. If a $100-\mathrm{mm}$ cigarette is tested, then an $85-\mathrm{mm}$ cigarette of the same brand and type should have emission factors which are approximately proportional to the reduced length and mass of tobacco.

Among the cigarettes with the largest market shares in California, almost all are filtered and many are mentholated. All of the types of Kool and Salem brand cigarettes are mentholated. The most popular types of Benson and Hedges cigarettes are also mentholated (FTC, 1992).

To select the cigarette brands and types for testing, market shares and types of cigarette, as presented in Table 5.1, were considered. Market shares were first considered separately for men and women since there was some difference in brand preference. Marlboro accounts for one-third or more or the market share for the California population for both men and women and it was clear that it should be included. For men, Camel, Winston and Benson and Hedges, in that order, are the next three most popular brands. For women, the next three most popular brands, in decreasing order, are Benson and Hedges, Winston and Salem. For men and women combined, Marlboro, Camel, Benson and Hedges, Winston and Salem account for $62.6 \%$ of the California market. These five brands include the top four for men and the top four for women.

There are also some data available on brand preference for certain ethnic categories in California (H. Johnson, CARB, personal communication). These are presented in Table 5.2. For the Hispanic, Asian/South Pacific Islander and American Indian ethnic categories, Marlboro, Camel, Benson and Hedges and Winston are among the most favored brands. For the African-American category, however, Newport and Kool have the largest percentages for brand preference, followed by Benson and Hedges, Marlboro and Salem. Marlboro, Camel, Benson and Hedges, Winston and Salem are among the top five favored brands for both men and women and most ethnic categories, and, are thus good candidates for selection. The most popular brand among the African-American group is Newport, which is not included in the top five above. This, however, is a mentholated cigarette and the top five brands listed above include two cigarette brands for which the mentholated type is the most popular - Benson and Hedges and Salem. 
Table 5.1. Brands of cigarettes purchased by California smokers over 18 years of age."

\begin{tabular}{|c|c|c|c|c|}
\hline \multicolumn{5}{|c|}{ Percentage of Market Share } \\
\hline Brand $d^{b .}$ & Men & Women & Totalc. & Types Available $^{\mathrm{d} .}$ \\
\hline Marlboro & 44.5 & 32.0 & 38.9 & REG, LT, MEN, MEN-LT, ULT-LT \\
\hline Camel & 10.5 & 2.8 & 7.1 & REG, REG/NF, IT, ULT-LT \\
\hline Benson \& Hedges & $\overline{4.5}$ & 9.8 & 6.9 & REG, LT, MEN, MEN-LT, ULT-LT, ULT-LT/MEN \\
\hline Winston & 7.1 & 4.6 & 6.0 & REG, LT, ULT-LT \\
\hline Salem & 2.8 & 4.7 & 3.7 & MEN, MEN-LT, MEN/ULT-LT, MEN-LT/SLIM \\
\hline Kool & 3.6 & $\overline{3.1}$ & 3.4 & MEN, MEN-NF, MEN-LT, MILD-MEN, DLX-MEN \\
\hline Pall Mall & $\overline{3.8}$ & 1.9 & 2.9 & REG, REG/NF, LT, LT/NF \\
\hline Newport & 2.6 & 2.8 & 2.7 & MEN, MEN-LT, MEN-LT/SLIM, REG \\
\hline Other & 20.5 & 38.3 & 28.4 & - \\
\hline
\end{tabular}

a. Based on data reported by Pierce, et al., 1991; 1992.

b. Manufacturers of brands: Marlboro - Phillip Morris; Camel - Camel; Benson and Hedges - Benson and Hedges; Winston - R.J. Reynolds Co.; Salem - R.J. Reynolds Co., Kool - Brown and Williamson Co., Pall Mall - American Tobacco Co.

c. Percentages are population weighted.

d. Types are categorized regardless of length. In the table, all types are filtered unless designated NF (non-filtered). REG (regular) is used here to designate cigarettes that are not specially flavored, light or ultra-light, regardless of length; MEN = mentholated; LT = light; ULT-LT = ultra-light, DLX = deluxe.

Table 5.2 Brand preferences of various ethnic categories in California as percent citing.

\begin{tabular}{|l|c|c|c|c|c|}
\hline Brand & White & $\begin{array}{c}\text { African- } \\
\text { American }\end{array}$ & Hispanic & $\begin{array}{c}\text { Asian/Pacific } \\
\text { Islanders }\end{array}$ & $\begin{array}{c}\text { American } \\
\text { Indian }\end{array}$ \\
\hline Marlboro & 39.8 & 10.0 & 59.7 & 42.9 & 36.4 \\
\hline Camel & 7.2 & - & 4.7 & 5.4 & 8.5 \\
\hline Winston & 6.3 & - & 7.4 & & 3.5 \\
\hline Benson \& Hedges & 6.3 & 12.0 & 5.9 & 11.2 & 3.2 \\
\hline Newport & & 23.5 & & & \\
\hline Kool & & 15.5 & & 2.9 & \\
\hline Salem & & 8.5 & 4.2 & 5.4 & \\
\hline
\end{tabular}

The second factor considered in selecting cigarettes was type within a brand category (e.g., regular, light, ultra-light, menthol and various combinations of these). The California survey does not contain information on market shares for the sub-categories. Since Kool and Benson and Hedges are included in the brands identified above as possible candidates for study, two mentholated cigarette types were included. Since Marlboro accounts for the greatest percentage of market share for both men and women, we decided that the sixth cigarette should be selected from among the remaining types of Marlboro cigarettes. A light cigarette was selected for testing and comparison to the regular type. 
According to Dr. Chortyk (U.S. Dept. of Agriculture, Tobacco Safety Research Unit, Agricultural Research Service, personal communication), for the light and ultra-light cigarettes, the major determinant of sidestream emissions is the weight of the tobacco burned. Dr. Chortyk also stated that two methods are used to reduce the tar content in light and ultra-light cigarettes: 1) the use of reconstituted tobacco (reconstituted tobacco includes less desirable but lower tar-producing tobacco leaf parts, such as stems); and, 2) the addition of perforations in the filter overwrap to allow more air to enter during puff drawing. The effect of both of these measures on sidestream emissions is expected to be much less than on mainstream smoke.

The question also arises as to whether the selected brands and types of cigarettes will provide a reasonable estimate of the variability in emission factors across many brands. There is only limited information on the variability of emissions among brands of cigarettes. For mainstream smoke, tar, nicotine and $\mathrm{CO}$ yields per cigarette generally range within a factor of three or less among the cigarettes listed in Table 5.1 (FTC, 1992). For specific VOCs, emission factors for mainstream smoke can vary over more than an order of magnitude among different brands and types (e.g., Schlitt and Knoppel, 1989; Fisher, et al., 1989).

There is some evidence which indicates that the variability in emission factors for sidestream smoke among different cigarette types (i.e., low and high tar) is less than for mainstream smoke. For example, Schlitt and Knoppel (1989) reported aldehyde emission factors for sidestream smoke from a "light" filter cigarette and a "strong" unfiltered cigarette. For formaldehyde, the emission factors were 2,200 and 2,300 mg/cig for the light and strong cigarettes, respectively. Emission factors for acetaldehyde were $4,700 \mathrm{mg} / \mathrm{cig}$ (light, filtered) and $6,100 \mathrm{mg} / \mathrm{cig}$ (strong, unfiltered), less than a two-fold difference. For mainstream smoke, the investigators found about a factor of six variation among the emission factors for the same two compounds. For acrolein, the emission factors varied by a factor of 12 for mainstream smoke, while for SS the emission factors were virtually identical.

Brunnemann, et al. (1977) reported SS emission factors for four different $\mathrm{N}$-nitrosamines that varied within a factor of three (with one exception) among four brands. For phenol and the cresols, the variation in SS emission factors among brands appears to be somewhat larger than for the aldehydes and $\mathrm{N}$ nitrosamines. For example, Chortyk and Schlotzhauer (1989) reported emission factors for phenol and the three cresol isomers for a number of U.S. low-tar filter cigarettes which varied by four to seven fold. There is some indication that SS emission factors for phenol and cresols emitted by low tar cigarettes are positively correlated with tar values for mainstream smoke (Chortyk and Schlotzhauer, 1989). However, the correlation was not very high. For the 20 cigarettes tested, the values of $\mathrm{r}^{2}$ for the correlation between the emission factors for phenol and the 3 cresols with tar in mainstream smoke ranged from 0.06 to 0.44 . That is, at best, the tar values could account for only $44 \%$ of the variance in the emission factors. Some of 
the variability was also probably due to differences in the lengths of the cigarettes and the weights of tobacco smoked.

Based on all of the information discussed above and discussions with the CARB staff, the following commercial cigarette brands were selected for testing:

- Benson \& Hedges, filter, mentholated

- Camel, non-filter

- Marlboro, filter

- Marlboro, filter, light

- Salem, filter, mentholated

- Winston, filter

The selected brands represent $62.5 \%$ of the market share (population weighted) of cigarettes sold in California in 1990 and include filtered, non-filtered and mentholated cigarettes. Four different cigarette manufacturers are represented. Each of the cigarette brands was assigned a letter code which is used in the remainder of this report.

\subsubsection{Protocols for Cigarettes and Smoking}

The commercial cigarettes used in the experiments were purchased in stores in Walnut Creek, $\mathrm{CA}$, in the fall and winter of 1993. The 1R4F reference cigarettes were obtained from the Tobacco and Health Research Institute, University of Kentucky, Lexington, KY. All cigarettes were conditioned for at least 48 hours prior to smoking at $60 \%$ relative humidity and room temperature in a dessicator over a solution of saturated sodium bromide. The average weight of tobacco per $\mathrm{cm}$ was determined by cutting off the butt section of six equilibrated cigarettes and weighing the tobacco in the remaining portion (Chortyk and Schlotzhauer, 1989). The mass of the cigarette tobacco smoked was calculated from the mass of the tobacco per unit length (average from six cigarettes without the filter and the paper), and the actual smoked length of the cigarette. The total length, the filter overwrap length, the standard smoked length and the mass of tobacco per $\mathrm{cm}$ length are presented in Table 5.3 for each of the cigarettes. The standard smoked length for filter cigarettes is the total length minus the butt length defined as the filter overwrap length plus $0.3 \mathrm{~cm}$; for the non-filtered cigarette, the standard smoked length is the total length minus a butt length of $2.3 \mathrm{~cm}$ (ISO, 1986). Cigarettes were machine smoked for both SS and ETS generation using standard protocols, i.e., a smoking cycle of one puff per minute of $35-\mathrm{cm}^{3}$ volume and 2 -sec duration. 
Table 5.3. Cigarette dimensions and tobacco weights per unit length.

\begin{tabular}{|l|c|c|c|c|}
\hline Cigarette & $\begin{array}{c}\text { Total Length, } \\
\mathbf{c m}\end{array}$ & $\begin{array}{c}\text { Overwrap Length, } \\
\mathbf{c m}\end{array}$ & $\begin{array}{c}\text { Standard } \\
\text { Smoked Length, } \\
\mathbf{c m}\end{array}$ & $\begin{array}{c}\text { Mass } \\
\text { Per Unit Length, } \\
\mathbf{m g} / \mathbf{c m}\end{array}$ \\
\hline A & 7.9 & 2.3 & 5.3 & $115.5 \pm 0.2$ \\
\hline B & 8.3 & 3.1 & 4.9 & $117.1 \pm 0.6$ \\
\hline C & 6.9 & - & 4.6 & $116.9 \pm 4.0$ \\
\hline D & 8.4 & 2.5 & 5.6 & $113.7 \pm 1.4$ \\
\hline E & 9.9 & 3.1 & 6.5 & $120.4 \pm 1.3$ \\
\hline F & 9.9 & 3.2 & 6.4 & $113.0 \pm 0.4$ \\
\hline 1R4F & 8.3 & 3.2 & 4.8 & $135.0 \pm 2.2$ \\
\hline
\end{tabular}

\subsection{Environmental Chamber}

The LBL Environmental Chamber, which was used for the experiments with ETS, is designed for investigations of emissions of pollutants from indoor sources under simulated, controlled indoor environmental conditions. This room-sized chamber, shown schematically in Figure 5.1, encloses a volume of $20 \mathrm{~m}^{3}$ with interior dimensions of $3.65 \mathrm{~m}$ (length) $\times 2.44 \mathrm{~m}$ (width) $\times 2.23 \mathrm{~m}$ (height). The walls, floor and ceiling are insulated with a $10-\mathrm{cm}$ layer of high-density polyurethane foam. All interior surfaces are clad with stainless steel. The door and interior seams are sealed with silicone gasket material. The synthetic materials used in construction of the chamber were selected, in part, for their low emissions of voCs.

The chamber is equipped with a single-pass ventilation system. Inlet air is drawn from outside the laboratory building by a variable-speed blower and passes through a filter assembly containing a coarse filter, 12 charcoal filters, and a HEPA (high efficiency particle air) filter, in series. The desired dew-point and dry bulb temperatures of the inlet air are established by a pre-heater, a humidifier, a chiller coil, and a re-heater in the air-handling system. The volumetric flow rate of air is monitored with a turbine flowmeter located downstream of the filter pack. Air enters the chamber through a diffuser positioned high at one end of a long wall and exits the chamber through an outlet located low at the opposite end of the same wall. Air is exhausted outdoors. Ventilation rates can be varied from about 0.1 to 10 air changes per hour (ach) or the chamber can be operated in static mode (i.e., the only air exchange is due to infiltration of air through the leakage pathways). At 1 ach, the variation was \pm 0.02 ach over a one week period. Air temperature in the chamber can varied from about $15^{\circ} \mathrm{C}$ to $30^{\circ} \mathrm{C}$ and held within $\pm 1{ }^{\circ} \mathrm{C}$ over a period of a week. Relative humidity can also be varied but is usually held at about $50 \% \pm 1.5 \%$. Prior to the chamber experiments, the interior surfaces of the chamber were washed with an alkaline cleaning solution, thoroughly rinsed and dried. 

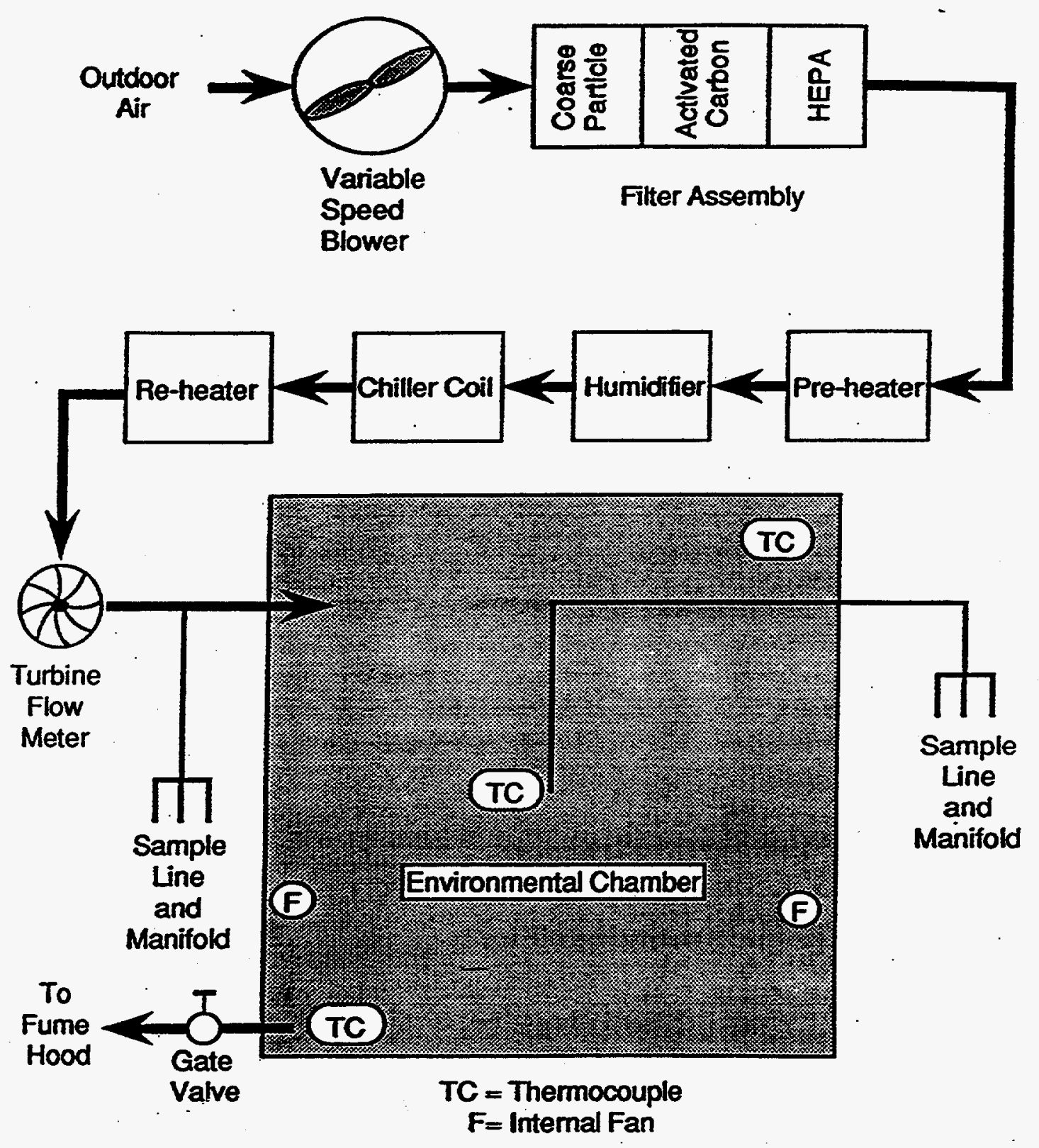

Figure 5.1. Schematic diagram showing a cross sectional view of the environmental chamber. 


\subsection{Protocols for the Environmental Chamber Experiments}

For measurements of emission factors, the chamber was operated in a static mode, that is, with a minimum air exchange rate. This mode of operation was required to maintain sufficiently high concentrations of ETS for the sampling and analyses of the pollutants over the 4-hour sampling period and to allow sufficient time for the aging of the smoke without removal by ventilation. If, for example, the chamber was operated at 0.5 air changes per hour, then, after 4 hours, approximately $94 \%$ of the original mass of ETS would be removed. Operation of the chamber in a static mode was not expected to affect the values of the emission factors.

The air temperature of the chamber was established and maintained by controlling the temperature of the laboratory in which the chamber is located. During each experiment, the air temperature in the chamber was periodically monitored at three locations with type $T$ thermocouples. The thermocouples were positioned in the chamber near the air inlet, the air exhaust and the mid-point. The readings from the three thermocouples were averaged to obtain an average air temperature. The air temperature in the laboratory was also monitored. All thermocouples were calibrated against a precision thermometer prior to the study.

The humidity of the chamber was adjusted, if necessary, by evaporating water in the chamber prior to the start of an experiment. This was necessary because of the static operating mode. The dew-point temperature of the air in the chamber was monitored periodically with a chilled-mirror dew-point hygrometer (Model 911 Dew-All, EG\&G, Inc.). This instrument was calibrated by the manufacturer prior to the study. Atmospheric pressure in the chamber was continuously monitored with a pressure transducer readable to one torr (Model PDCP-20A-230, Columbia Research Laboratories, Inc.). The calibration of the transducer was checked against a mercury barometer.

The analog output signals from the thermocouples, the dew-point hygrometer and the pressure transducer were continuously sampled throughout the experiment with a data-acquisition system (Series 500 , Keithly/DAS) at a rate of five points per minute. Data collection, processing and recording were controlled with a personal computer running a commercial data-acquisition software program (Labtech Notebook, Ver. 6.01, Laboratory Technologies Corp.). Average parameter values for consecutive fifteenminute intervals were recorded on a hard disk and backed-up on diskettes.

A 20-watt fluorescent lamp (Model 9010B, Lights of America, Walnut Creek, CA) was mounted in the chamber on the walls. In a preliminary experiment, the lamp was operated for 5.5 hours while the temperatures in the chamber and the laboratory in which the chamber is located were monitored. During that period, the temperature in the chamber did not rise. Consequently, all of the chamber experiments were run with the lamp on to simulate lighting in an indoor environment. 
Table 5.4 presents a summary of the average chamber temperature and relative humidity for each experiment. The average temperatures and relative humidities are typical for indoor environments. The relatively small variations in these parameters among the experiments would not be expected to affect the values of the emission factors.

Table 5.4. Average temperatures and relative humidities for the environmental chamber experiments.

\begin{tabular}{|c|c|c|c|}
\hline Experiment No. & Cigarette & Average Temperature, ${ }^{\circ} \mathbf{C}$ & Average Relative Humidity, \% \\
\hline 1 & A & $23.8 \pm 0.3$ & $46.0 \pm 0.8$ \\
\hline 2 & $\mathrm{~A}^{\mathrm{a}}$ & $24.1 \pm 0.2$ & $42.5 \pm 0.7$ \\
\hline 3 & $\mathrm{D}$ & $23.5 \pm 0.2$ & $43.1 \pm 1.2$ \\
\hline 4 & $\mathrm{~B}$ & $22.7 \pm 0.4$ & $49.0 \pm 1.8$ \\
\hline 5 & $\mathrm{~F}$ & $23.9 \pm 0.2$ & $47.9 \pm 1.9$ \\
\hline 6 & $\mathrm{E}$ & $23.6 \pm 0.3$ & $49.4 \pm 1.5$ \\
\hline 7 & $\mathrm{C}$ & $23.2 \pm 0.1$ & $44.0 \pm 0.4$ \\
\hline 8 & 1R4F & $23.2 \pm 0.2$ & $49.2 \pm 1.0$ \\
\hline
\end{tabular}

a. Duplicate chamber experiment.

Six small variable-speed fans, mounted on the four walls of the chamber, at one-third or two-thirds (alternating) of the height of the chamber wall (two fans were positioned on each of the long walls) were operated continuously to establish and maintain uniform mixing in the chamber.

The air exchange rate for the chamber in static mode was determined by releasing a small aliquot of $\mathrm{SF}_{6}$, an inert gas, into the chamber and mixing the air. Samples of air were then withdrawn at regular intervals and analyzed with a gas chromatograph (Model HP 5890A, Hewlett-Packard Co.) equipped with a $60 / 80$ Chromasil 310 column and an electron-capture detector. The air exchange rate was then determined from linear regression analysis of the logarithm of the concentration of $\mathrm{SF}_{6}, \ln \mathrm{C}\left(\mathrm{SF}_{6}\right)$, versus time:

$$
\ln \mathrm{C}\left(\mathrm{SF}_{6}\right)=\ln \mathrm{C}\left(\mathrm{SF}_{6}\right)_{0}-\lambda \mathrm{t}
$$

where $\mathrm{C}\left(\mathrm{SF}_{6}\right)_{0}$ is the concentration of $\mathrm{SF}_{6}$ at time zero, $t$ is time, and $\lambda$ is the air exchange rate. The air exchange rate for the chamber in static mode was determined to be $0.005 \mathrm{~h}^{-1}$. The act of sampling induced an additional $0.024 \mathrm{~h}^{-1}$ air exchange over the period of the experiment. The air exchange rate for the chamber experiments and for the calculation of the emission factors was estimated as the sum of the background air exchange rate plus the air exchange due to sampling and subsequent infiltration of laboratory air to replace the removed air, i.e., $0.029 \mathrm{~h}^{-1}$. During each chamber experiment, the 
concentration of carbon monoxide ( $\mathrm{CO}$ ) was continuously monitored as a check on the total air exchange rate for the chamber to ensure that there was no unexpected air infiltration. Carbon monoxide is very chemically stable and is not lost through deposition on walls. The concentration of $\mathrm{CO}$ did not change significantly during any of the experiments.

For each experiment, sidestream emissions from three cigarettes were generated in the chamber. The cigarettes were smoked in sequence, each for 8 to 9 minutes, using a single-port smoking machine (ADL/II Smoking System, Arthur D. Little, Inc.) attached to a twelve-port cigarette holder. The holder, designed and constructed at LBL, was programmable and had auto-igniting and extinguishing capabilities. A standard smoking cycle of one puff per minute of $35-\mathrm{cm}^{3}$ volume and 2-second duration was used. Cigarettes were smoked to a standard butt length and then mechanically extinguished by the holder. Nonfilter cigarettes were smoked to a butt length of $23 \mathrm{~mm}$; filter cigarettes were smoked to the length of filter overwrap plus $3 \mathrm{~mm}$, as specified by ISO (1986) and by the U.S. Federal Trade Commission.

The smoking machine was positioned toward one corner of the chamber. Mainstream smoke was vented to the exterior of the chamber so that only sidestream smoke contributed to the simulated ETS. Through observation we could see that the smoke plume was quickly mixed by the fans. Sample collection was initiated (defined as $\mathrm{t}=0$ ) immediately after the third cigarette was extinguished. All samples were collected near the center of the chamber. A summary of the sampling protocols for each class/species is presented in Table 5.5. Figure 5.2 presents a schematic diagram of the environmental chamber showing the locations and configurations of all of the samplers and their flow rates.

Table 5.5. Summary of the sampling protocols and analysis methods for ETS measurements. ${ }^{2}$

\begin{tabular}{|c|c|c|c|c|c|}
\hline Compound or Class & $\begin{array}{c}\text { Collection } \\
\text { Method } \\
\end{array}$ & $\begin{array}{c}\text { No. of } \\
\text { Samples per } \\
\text { Experiment }^{\text {b. }}\end{array}$ & $\begin{array}{l}\text { Sampling } \\
\text {. Rate, } \\
\text { mL/min }\end{array}$ & $\begin{array}{c}\text { Sampling } \\
\text { Duration, } \\
\text { min }\end{array}$ & Analysis Method \\
\hline N-Nitrosamines & $\begin{array}{c}\text { Thermosorb/N } \\
\text { cartridge }\end{array}$ & 1 & 4,000 & 250 & GC-TEA \\
\hline Aldehydes & $\begin{array}{c}\text { Silica-DNPH } \\
\text { cartridge }\end{array}$ & $2^{c}$ & 255 & 100 & HPLC-UV \\
\hline VOCs & $\begin{array}{l}\text { Multisorbent } \\
\text { cartridge }^{d .}\end{array}$ & $4^{\mathrm{e}}$ & 36.5 & 60 & GC/MS \\
\hline Nicotine & $\begin{array}{c}\text { XAD-4 } \\
\text { sorbent tube }\end{array}$ & 1 & 2,000 & 250 & GC-NPD \\
\hline Particulate Matter & Teflon filter & 1 & 4,000 & 250 & Microbalance \\
\hline
\end{tabular}

a. $\mathrm{NO}_{\mathrm{x}}$ and $\mathrm{CO}$ were continuously monitored.

b. Chamber blanks were also collected prior to each chamber experiment and analyzed.

c. Two samples were collected sequentially during the first and last 100 minutes of the 250 -minute experiments.

d. Tenax-TA, Ambersorb XE-340 and activated charcoal, in series.

e. Four samples collected sequentially. 


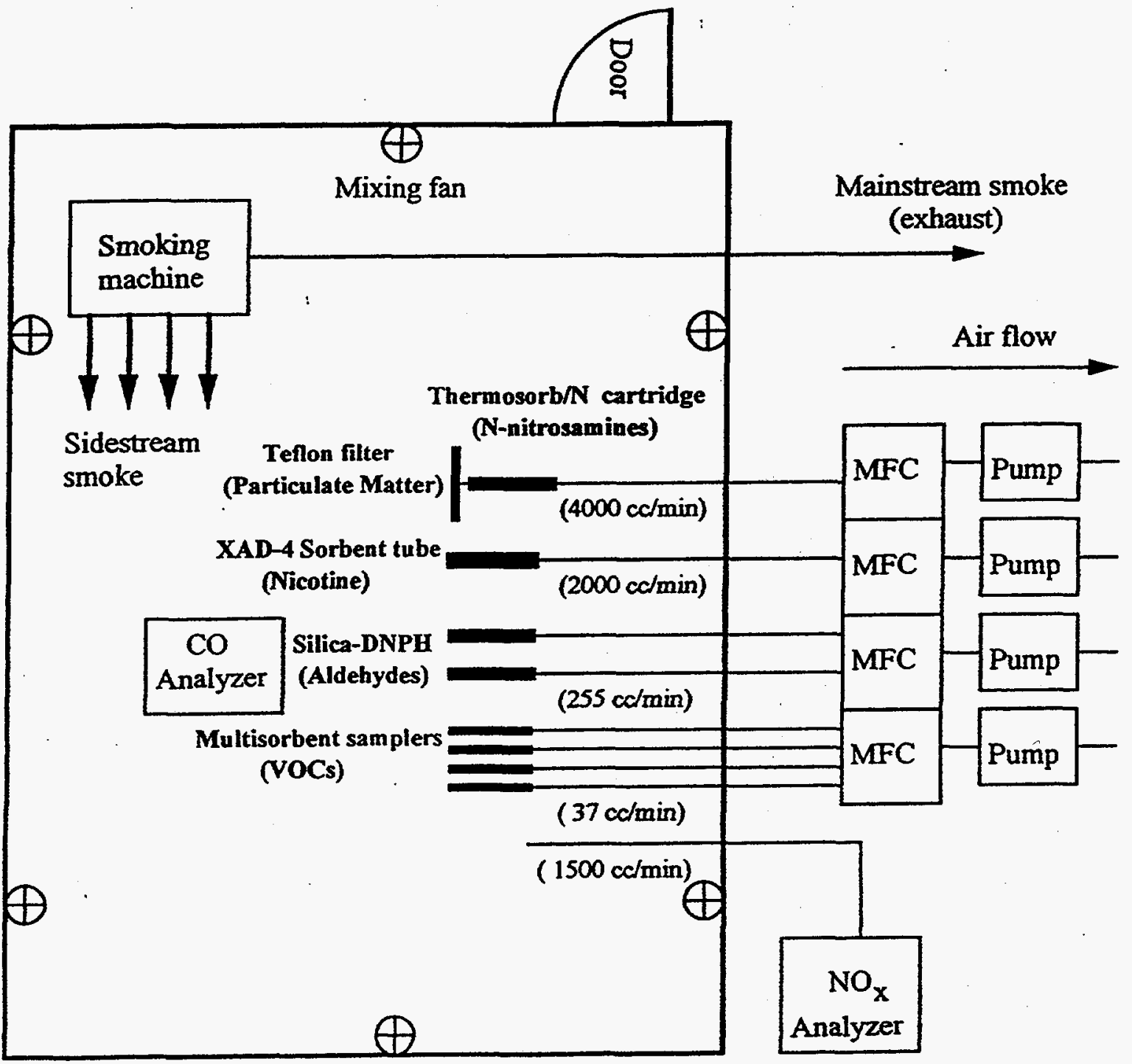

Figure 5.2. Schematic diagram showing the locations; configurations, and sampling rates for samples collected in the environmental chamber experiments. MFC $=$ mass flow controller. 


\subsection{Apparatus and Experimental Protocols for Measurements of Sidestream Smoke}

Samples of sidestream smoke (SS) were collected from the sidestream sampling apparatus (SSA) shown in Figure 5.3. This apparatus which has an internal volume of $225 \mathrm{~mL}$ is similar to that used by Brunnemann and Hoffmann (1974, 1978). The apparatus consists of a glass chamber with inlets for a cigarette and for air intake positioned at one end, and with a side arm for sample collection, located at a 90degree angle with respect to the cigarette. Unlike the apparatus used by Brunnemann and Hoffmann, it did not have a water-jacket for cooling. The jacket was omitted based on the recommendation of Dr. Brunnemann (personal communication). Elimination of the additional cooling helped to reduce some of the condensation of cigarette smoke components on the inner surfaces of the apparatus. An external smoking machine (ADL/I Smoking System, Arthur D. Little, Inc.) smoked a cigarette that was inserted into the SSA. Sidestream smoke samples were drawn from the side arm (positioned horizontally with respect to the benchtop) at a $1.5 \mathrm{~L} / \mathrm{min}$ flow rate throughout the smoking. Sampling started with insertion of the ignited cigarette into the SSA. The cigarette was smoked for 10 to 11 minutes (i.e., 10 to 11 puffs). Sampling was stopped 8 seconds after removal of the cigarette. One cigarette was smoked for the measurement of $\mathrm{N}$ nitrosamines, one for the aldehydes and VOCs, and one for nicotine and particulate matter. Table 5.6 presents a summary of the sampling protocols for the SS measurements.

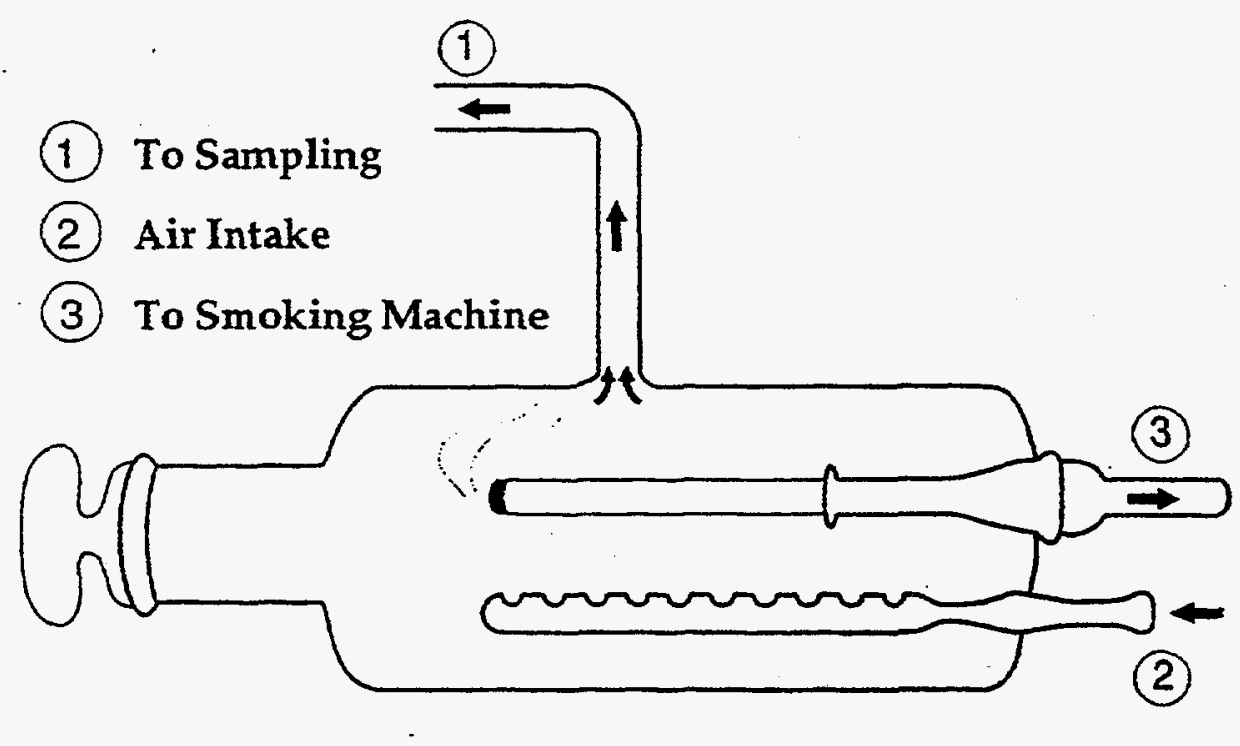

Figure 5.3. Schematic diagram of the sidestream apparatus. 
Table 5.6. Summary of the sampling protocols and analysis methods for SS measurements. ${ }^{2}$

\begin{tabular}{|c|c|c|c|c|c|}
\hline $\begin{array}{l}\text { Compound } \\
\text { or Class }\end{array}$ & Collection Method & $\begin{array}{c}\text { No. of } \\
\text { samples } \\
\text { collected }^{\mathbf{b}} \text {. }\end{array}$ & $\begin{array}{c}\text { Sampling } \\
\text { Rate, } \\
\mathrm{mL} / \mathrm{min}\end{array}$ & $\begin{array}{c}\text { Sampling } \\
\text { Duration, } \\
\text { min }\end{array}$ & $\begin{array}{l}\text { Analysis } \\
\text { Method }\end{array}$ \\
\hline N-Nitrosamines & Thermosorb/N cartridge ${ }^{\mathrm{c}}$ & 1 & 1,500 & $10-11$ & GC-TEA \\
\hline Aldehydes & Silica-DNPH cartridge ${ }^{\mathrm{d}}$ & 1 & 30 & $10-11$ & HPLC-UV \\
\hline VOCs & Multisorbent cartridge $e^{d}$ & 1 & 1 & $10-11$ & GC/MS \\
\hline Nicotine & XAD-4 sorbent tube $e^{e .}$ & 1 & 20 & $10-11$ & GC-NPD \\
\hline Particulate Matter & $\begin{array}{l}\text { Teflon-coated glass-fiber } \\
\text { filtere. }\end{array}$ & 1 & 1,480 & $10-11$ & $\begin{array}{c}\text { Electronic } \\
\text { Balance }\end{array}$ \\
\hline
\end{tabular}

a. One cigarette was smoked for the measurement of N-nitrosamines, one for the aldehydes and VOCs, and one for nicotine and particulate matter; a second cigarette was smoked for each of the duplicate samples.

b. For each brand, a blank was also collected and analyzed.

c. Also recovered from the SSA walls and the particulate filter.

d. 1:1 dilution with nitrogen for sampling.

e. Also recovered from the SSA walls.

\subsection{Sampling and Chemical Analysis for N-Nitrosamines in Tobacco Smoke}

Four volatile N-nitrosamines (VNA) were targeted for measurement in both ETS and SS: N-nitrosodimethylamine (NDMA), N-nitrosodiethylamine (NDEA), N-nitrosopyrrolidine (NPYR), and N-nitrosomorpholine (NMPH). As part of this study, we developed a more convenient sampling method using solid-phase cartridges which avoids many of the drawbacks of the liquid-phase sampling used by other investigators for similar applications.

Sampling. ETS samples were drawn from near the center of the environmental chamber. Sampling started immediately after the third cigarette was smoked and continued for a period of $250 \mathrm{~min}$ at a flow rate of $4 \mathrm{~L} / \mathrm{min}$. The sampling line, shown schematically in Figure $5.4 \mathrm{~b}$, contained an open-face Teflon filter (Gelman Sciences) for particles followed by a Thermosorb/N cartridge (Thermedics Detection, Inc.), a mass-flow-controller and a vacuum pump.

Sidestream smoke samples were drawn from the SSA through the side arm at a $1.5 \mathrm{~L} / \mathrm{min}$ flow rate throughout the smoking. The sampling line for SS consisted of an in-line ascorbic acid-impregnated Cambridge filter ${ }^{*}$ to remove particles, followed by the Thermosorb/N cartridge and a mass-flow controller and a vacuum pump, as shown schematically in Figure 5.4a.

\footnotetext{
* Cambridge filters have traditionally been used for sampling tobacco smoke because of their efficiency in trapping small particles and their low resistance to air flow. They are composed of a glass-fiber pad held together with an acrylic binder. For sampling the highly concentrated SS emissions, the Cambridge filter was impregnated with ascorbic acid to prevent artifactual formation of N-nitrosamines from NOx and amines in the SS.
} 
In addition to the SS samples collected on the filter and cartridge, the interior walls of the SSA were washed with solvent to recover condensate-trapped VNA. This was done because the SS mixture of gases and particles emitted from the burning cone of the cigarette in the SSA is highly concentrated and there is a temperature gradient of about $900^{\circ} \mathrm{C}$ between the burning cone and the apparatus walls. These conditions lead to substantial condensation of SS as oily deposits in the apparatus, on the Cambridge filter, and in the sampling line. Most of the colored (yellowish and dark brown) SSA condensates were found on the inner wall just above the burning cone. Although the amount of condensate deposition can be minimized by keeping the upper wall of the SSA away from the burning cone, it cannot be entirely eliminated. Brunnemann observed similar condensation and suggested combining the condensate and the filter deposit for analysis (personal communication).

Analysis. To extract and clean-up the ETS and SS collected on a Thermosorb/N cartridge, the sample-loaded cartridge was coupled to an alumina-B Sep-Pak. An aliquot of an internal standard (70 to $80 \mathrm{ng}$ of NDEA) was injected into the cartridge. A $1.5-\mathrm{mL}$ aliquot of methanol in dichloromethane (DCM) $(1: 2, \mathrm{v}: \mathrm{v})$ was then added to the cartridge. The sample, nitrosating inhibiting agent,"* and internal standard were allowed to dissolve in the solvent for a minute. A $5-\mathrm{mL}$ aliquot of $10 \%$ chloroform in DCM was then added to the inlet of the cartridge, and pressurized air was used to elute the solution from the cartridge onto the coupled alumina-B Sep-Pak. The cartridge and Sep-Pak were then separated, and the cleaned extract was recovered from the Sep-Pak using air pressure. The extract was concentrated to a volume of 250 to 500 $\mu \mathrm{l}$ in a rotary evaporator and an aliquot of this was analyzed on a gas chromatograph with a thermal energy analyzer detector (GC/TEA) (See below).

For VNA analysis of SS samples, three sub-samples (the SSA condensate, the filter extract and the Thermosorb/N cartridge extract) were recovered and analyzed separately for VNA. The condensate was recovered from the interior of the SSA by rinsing it with $1 \mathrm{~mL}$ of methanol followed by $5 \mathrm{~mL}$ of $10 \%$ chloroform in DCM. The SSA rinsings were combined, an aliquot of the internal standard (87 $\mathrm{ng}$ NDEA) was added, and the extract was loaded onto an alumina-B Sep-Pak for clean-up. The Sep-Pak was then eluted with $5 \mathrm{~mL}$ of $10 \%$ chloroform in DCM. No air pressure was required to recover the eluate from the Sep-Pak alone. The eluate was then reduced in volume to 250 to $500 \mu \mathrm{l}$ using a rotary evaporator, and an aliquot of this was analyzed by GC/TEA.

\footnotetext{
** The inhibiting agent is incorporated into the Thermosorb $\mathrm{N}$ cartridge by the manufacturer to prevent artifactual formation of $\mathrm{N}$ - nitrosamines during sampling. It is eluted with the sample.
} 
a.

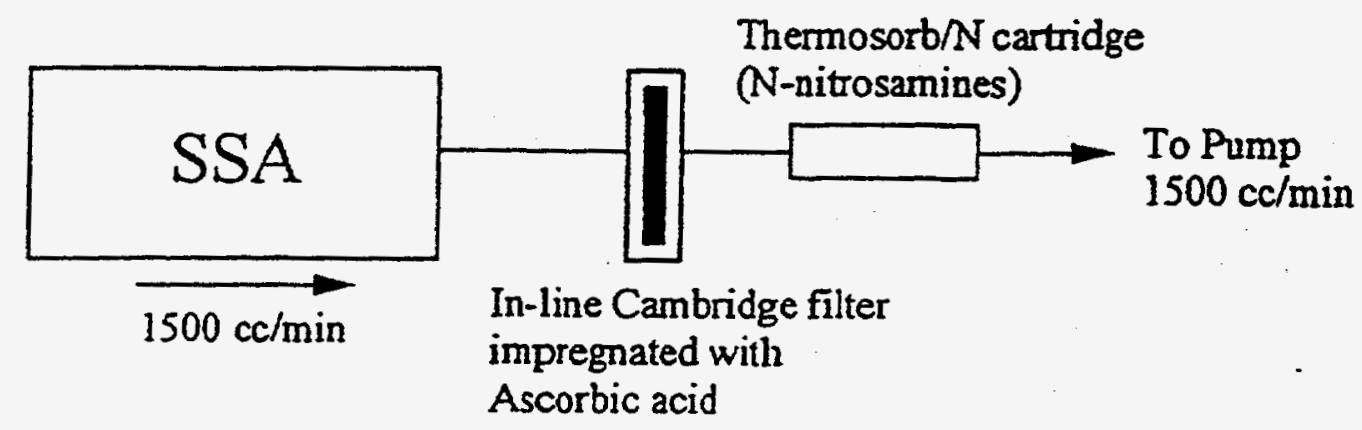

b.

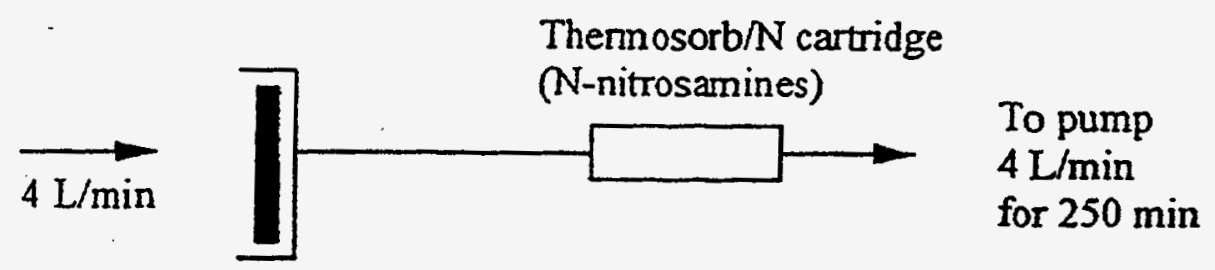

Open-face Teflon filter

Figure 5.4. Schematic diagrams of the sampling trains used for collecting N-nitrosamines in a.) SS and b.) ETS.

For analysis of VNA in the SS collected on the filter, an aliquot of the NDEA internal standard was added to the loaded filter, and the solvent was allowed to evaporate for a few minutes. The filter was then cut into 8 pieces with clean scissors and placed in a $25-\mathrm{mL}$ Erlenmeyer flask. Ten $\mathrm{mL}$ of $10 \%$ chloroform in DCM was added to the flask, and the flask was sonicated for 30 minutes. A portion of the filter extract was drawn up into a 5-mL glass syringe. The syringe was then coupled to an alumina-B Sep-Pak, and the eluate was collected as it came off the Sep-Pak. No air pressure was required for this. The remaining 4-5 $\mathrm{mL}$ of extract was drawn up into the glass syringe and added to the Sep-Pak for cleanup. Finally, the filter pieces in the flask were transferred to the glass barrel of the syringe, the Erlenmeyer was rinsed with additional solvent, and the rinsings were added to the syringe barrel, while the syringe was coupled to the Sep-Pak. After the rinsings drained through the Sep-Pak, the barrel of the syringe was used to gently squeeze the filter pieces to recover any remaining extract and pass it through the Sep-Pak. The 
combined eluates were then reduced in volume to 250 to $500 \mu \mathrm{l}$ and an aliquot was analyzed by GC/TEA. The VNA collected on the Thermosorb/N cartridge were recovered and cleaned up as described above. The cleaned samples from the condensate and the filter had more yellowish color than that from the cartridge.

A Hewlett Packard 5890A gas chromatograph (GC) equipped with a capillary DB-Wax column (30-m $\times$ 0.32-mm I.D. x 0.25 micron film thick fused-silica capillary column, J\&W Scientific, Inc.) was used for separation. A thermal energy analyzer ( TEA; Model 54, Thermedetec, Inc.), was used for detection. The GC-TEA interface was maintained at $225^{\circ} \mathrm{C}$, and the furnace of the TEA was operated at $550^{\circ} \mathrm{C}$. Oxygen flow to the TEA was regulated at $30 \mathrm{~mL} \mathrm{~min}^{-1}$, and ozone flow to the vacuum chamber was adjusted to 1.5 torr at $50^{\circ} \mathrm{C}$. Helium was used as the carrier gas, and 2- $\mu 1$ samples were injected for the analysis. The GC oven temperature program started at $50^{\circ} \mathrm{C}$ and was held for 2 minutes, then ramped at $8^{\circ} \mathrm{C}$ per minute for 15 minutes to $180^{\circ} \mathrm{C}$ where it was held for another 3 minutes. Under these separation conditions, the retention times of NDMA, NDEA, NPYR and NMPH were 4.2, 5.3, 10.8 and $11.7 \mathrm{~min}$, respectively.

Preliminary experiments showed that NDEA and NMPH were not present at measurable levels in the ETS or SS samples. NDEA was, therefore, selected as the internal standard for the analysis. It eluted between NDMA and NPYR with good resolution and peak shape.

No detectable levels of background VNA were found in Thermosorb/N cartridges, in alumina-B Sep-Paks or in the organic solvents. Standard compounds, solvents and cartridges were used as purchased from the manufacturers. N-nitrosamine standard compounds were purchased form the following suppliers; NDEA (98\%) from Pfaltz \& Bauer, NMPH (98\%) from Sigma Chemical Co., and NPYR (99\%) and NDMA (99\%) from Aldrich Chemical Co. Standard solutions of the $\mathrm{N}$-nitrosamines were prepared in $\mathrm{DCM}$ at $\mu \mathrm{g} / \mathrm{mL}$ levels and working solutions were prepared by appropriate dilution. Both the standards and liquid samples were kept in the dark and refrigerated when not in use. Refrigerated samples were brought to room temperature and sonicated for 5 minutes before use.

\subsubsection{Development of a Sampling and Analysis Method for N-Nitrosamines in Tobacco Smoke}

Airborne volatile $\mathrm{N}$-nitrosamines (VNA) are very difficult to measure reliably due to the potential for creation or loss of $\mathrm{N}$-nitrosamines during sample collection. Nitrogen oxides and free amines in an air sample can react to create new $\mathrm{N}$-nitrosamines as artifacts. Alternatively, false negative results can occur due to the degradation of $\mathrm{N}$-nitrosamines from exposures to ultraviolet light and to the breakthrough of the compounds from the sampling medium. 
The standard method for sampling VNA from SS tobacco smoke was developed in the 1970s and employs a trapping solution in several serially-connected impingers. Nitrosating inhibitors are added to the trapping solution to prevent the formation of artifact $\mathrm{N}$-nitrosamines. A commonly used nitrosating inhibitor is ascorbic acid (Mirvish, et al., 1972), which competes with the amines for nitrite, thus preventing the formation of artifact $\mathrm{N}$-nitrosamines. The most highly developed and validated method to collect VNA consists of a bubbler or impinger train containing an aqueous buffer solution ( $\mathrm{pH}=4.5$ citrate-phosphate) with $20 \mathrm{mM}$ ascorbic acid (Caldwell and Conner, 1990; Fischer and Spiegelhalder, 1989; Hecht, et al., 1983; Brunnemann and Hoffmann, 1978; Brunnemann, et al., 1980). This method is limited in air flow rate (especially for midget-impingers), and the impingers are not a convenient sampling method in terms of handling. Labor intensive sample preparation procedures, the fast degradation of aqueous ascorbic acid solution, and the degradation of $\mathrm{N}$-nitrosamines from exposure to ultraviolet light during sampling (a lesser problem in indoor environments) are several other drawbacks to the use of this method. Among the other VNA collection methods, wet traps such as $1 \mathrm{~N} \mathrm{KOH,} \mathrm{cold} \mathrm{traps} \mathrm{and} \mathrm{Tenax} \mathrm{traps} \mathrm{have} \mathrm{been} \mathrm{reported} \mathrm{with}$ their own limitations (Fine, et al., 1977; Cucco and Brown, 1981).

\subsubsection{Evaluation of Various Sampling Media}

In more recent years, dry sorbents packed in cartridges have been developed for sampling many airborne pollutants due to their ease of use in field and laboratory settings. In this project, we undertook some work to develop and validate a dry sorbent/cartridge sampling medium to collect artifact free $\mathrm{N}$ nitrosamines from tobacco smoke because of the clear advantages of this type of sampler. Initially we investigated alumina-B Sep-Paks spiked with ascorbic acid for this purpose. Aliquots of the standard solution of N-nitrosamines (NDMA, NDEA, NPYR and NMPH) were added to Sep-Paks and then the SepPaks were eluted with $5 \mathrm{~mL}$ of $10 \%$ chloroform in DCM and analyzed to determine recoveries of VNA.

We also investigated and compared recoveries of the VNA from a commercially available cartridge developed for sampling VNA in ambient outdoor air. Thermosorb/N have been specifically designed for the quantitative collection of N-nitrosamines in outdoor air or industrial workplaces (Fine, et al., 1993) but have not been validated for $\mathrm{N}$-nitrosamines in tobacco smoke. The cartridge contains two sorbent zones, the first of which selectively traps and removes amines from the incoming air and prevents nitrosamine formation by airborne nitrogen oxides, and the second of which contains a chemical nitrosating inhibitor system to prevent $\mathrm{N}$-nitrosamine formation following sample elution. Both sample handling and sample recovery are much more convenient from the solid sorbent than from the aqueous trapping solutions that have been used in impingers to trap N-nitrosamines for cigarette smoke. In addition, problems such as emulsion formation which are often encountered in liquid-liquid extractions are eliminated. The cartridges have a moderate sampling capacity ( $1500 \mathrm{ng}$ per cartridge); however, they can be connected in series to increase the total capacity of the sampling system. In view of these positive features, experimental work to 
test and validate a solid sorbent sampling method for N-nitrosamines in tobacco smoke (ETS and SS) was undertaken.

The efficiency of VNA desorption from Thermosorb/ $\mathrm{N}$ cartridges were evaluated by spiking aliquots of a standard mixture and then eluting the air-dried cartridge with $2 \mathrm{~mL}$ of methanol in DCM (1:2, $\mathrm{v} / \mathrm{v}$ ). Recovery of VNA from standard impinger solutions was also determined for comparison.

The 15-mL aqueous impinger solution was spiked with the standard solution at two different levels and extracted with $2 \times 3 \mathrm{~mL}$ of $10 \%$ chloroform in DCM. The organic layer was separated and passed through a column containing $5 \mathrm{~g}$ of anhydrous sodium sulfate (to eliminate water) and then through an alumina-B Sep-Pak column (for clean-up). Once all of the organic solvent was passed through, a 2-mL aliquot of 1:2 $(\mathrm{v} / \mathrm{v})$ methanol in DCM was added to the anhydrous sodium sulfate column and air pressure was applied to recover the VNA. Recoveries were poor when the methanol mixture was not used for the elution.

The results of these experiments are presented in Table 5.7. Recoveries were good (>90\%) from the alumina-B Sep-Paks, the Thermosorb/N cartridges and the impingers at the 95-ng spiking level. Recoveries from the impingers at the lower spiking level were poorer but still reproducible and acceptable.

Table 5.7 Percentage recovery of spiked VNA from different sampling media.

\begin{tabular}{|l|c|c|c|c|}
\hline & \multicolumn{4}{|c|}{ Percent Recovery } \\
\hline Spiked VNA & Sep-Pak & Thermosorb/N & Impinger & Impinger \\
\hline Mass spiked (ng): & 115 & 114 & 15 & 95 \\
\hline Number of trials: & 5 & 5 & 5 & 5 \\
\hline & & & & \\
\hline NDMA & $91 \pm 9$ & $93 \pm 2$ & $60 \pm 1$ & $81 \pm 5$ \\
\hline NDEA & $95 \pm 13$ & $95 \pm 7$ & $65 \pm 4$ & $93 \pm 3$ \\
\hline NPYR & $97 \pm 12$ & $103 \pm 4$ & $84 \pm 2$ & $91 \pm 3$ \\
\hline NMPH & $99 \pm 11$ & $94 \pm 5$ & $79 \pm 4$ & $91 \pm 4$ \\
\hline & & & & \\
\hline Average Recovery \pm Std. Dev. & $95 \pm 11$ & $96 \pm 5$ & $72 \pm 3$ & $89 \pm 4$ \\
\hline
\end{tabular}

The Sep-Paks looked particularly promising because both the sampling and sample clean-up steps could be accomplished with a single column. Also, the recovery of VNA using a relatively non-polar solvent mixture is a very significant advantage because it produces a much cleaner extract from a complex matrix like tobacco smoke. In addition, less sample handling should improve the overall precision of the 
analytical procedure. However, considerably more validation work would have been required to demonstrate artifact-free sampling, e.g., experiments in which $\mathrm{NO}_{\mathrm{x}}$ would be drawn through Sep-Paks loaded with precursor amines. Consequently, we focused on the Thermosorb/N cartridges which had been validated for artifact-free sampling under outdoor ambient conditions but not for tobacco smoke.

We next evaluated the breakthrough of VNA from the Thermosorb/N cartridges by pulling room air through a VNA-spiked cartridge for $250 \mathrm{~min}$ at a flow rate of $4 \mathrm{~L} / \mathrm{min}$. The flow rate, air sample volume and the level of VNA spiked were selected to approximate conditions expected for ETS samples in the chamber. A second Thermosorb/N cartridge was placed downstream of the spiked cartridge to collect any breakthrough. No detectable levels of VNA were found in the downstream cartridge as long as all of the solvent from the spiking solution was evaporated before drawing sampling air through the cartridge. Breakthrough was further evaluated by sampling VNA from both SS and ETS using two cartridges in series. Since no VNA was detected in the downstream cartridge in either case (spiked cartridge or real samples), we concluded that use of a single Thermosorb/N cartridge was justified for sampling ETS or SS.

\subsubsection{Development of a Sample Clean-up Method for VNA Collected on Thermosorb/N Cartridges}

There is one problem with the Thermosorb/N cartridge. The cartridge was designed so that the nitrosating inhibitor agent used to prevent artifact formation during sampling elutes from the cartridge along with the $\mathrm{N}$-nitrosamines during the elution step. The manufacturer intended this to prevent further artifact formation in the eluted sample (Thermedics Detection, Inc., personal communication). The manufacturer suggests that the extracts can be analyzed directly without a clean-up step. This may be acceptable for outdoor air samples. However, for a more complex matrix, such as tobacco smoke, the use of an additional clean-up process and/or a selective extracting solvent is essential.

In traditional bubbler or impinger methods, the VNA are extracted using DCM and subsequently cleaned on a packed alumina column (Brunnemann, et al., 1977; Brunnemann and Hoffmann, 1978; Fischer and Spiegelhalder, 1989). Initially, sample-loaded Thermosorb/N cartridges were eluted with methanol in DCM $(1: 2, v: v)$, and the eluants were passed through an alumina-B Sep-Pak to remove polar compounds. Although some polar compounds were retained, methanol eluted some of the polar compounds from the Sep-Pak. Analysis of these extracts contaminated the GC column and interfered with the analysis of subsequent samples. The problem (worse in the more concentrated SS samples than in ETS samples) was due to the polar compounds. Several different strategies were tried to minimize these interferences.

We attempted to develop a more selective extraction by using different solvents. The idea was to minimize the extraction of the polar interferences. Neither DCM nor chloroform could completely recover VNA although these solvents did leave most of the polar compounds on the clean-up column. Other non- 
polar solvents such as $n$-hexane were also not efficient for the extraction. Acetonitrile was an efficient solvent for extraction but required a significantly larger volume and also created problems with the GC separation. Other polar solvents, such as acetone, completely recovered the VNA but also extracted polar compounds.

Efforts were then made to eliminate polar interferences and methanol from the extracts using an additional off-line step in the sample clean-up procedure. The extracts in methanol were concentrated, loaded onto an alumina-B Sep-Pak, and air dried for 5 to 6 hours to eliminate methanol (care was taken not to expose the sample to light). Since we found breakthrough of VNA when air is passed through a cartridge containing any residual solvent, we did not attempt to dry the solvent by purging the Sep-Pak with gas. The air-dried Sep-Pak was eluted with $10 \%$ chloroform in DCM to recover VNA, leaving the interfering polar compounds on the Sep-Pak. The eluate was then reduced in volume using a rotary evaporator. Although this procedure yielded a cleaner extract, significant losses of some VNA were observed. These may have been due to volatilization during the multi-concentration steps and/or to the decomposition of VNA during the time consuming solvent drying process. A modified clean-up procedure was then developed which solved the polar interference problem and gave good recoveries of the VNA.

In the modified extraction-clean-up procedure, the sample-loaded cartridge was coupled to an alumina-B Sep-Pak. This procedure was described fully in Section 5.6. In brief, an internal standard was injected into the cartridge. Methanol in DCM (1:2, v:v) was added to the cartridge, and the sample, nitrosating inhibiting agent, and internal standard were allowed to dissolve in the solvent for a minute. A 5 $\mathrm{mL}$ aliquot of $10 \%$ chloroform in DCM was then added to the cartridge and pressurized air was used to elute the solution from the cartridge onto the coupled Sep-Pak. The sampling cartridge was then separated from the Sep-Pak, and the cleaned extract was recovered from the Sep-Pak using air pressure. The extract was concentrated in a rotary evaporator, and an aliquot of this was analyzed on a GC with a TEA detector. Extracts prepared by this procedure were cleaner than those obtained with other procedures and no chromatographic problems were observed.

N-Nitrosodiethylamine was selected for use as an internal standard for the analysis of VNA because it was not found at detectable levels ( $<5 \mathrm{ng} / \mathrm{cig}$ ) either in ETS or SS. Using the method described above, recovery of spiked NDEA was $97.6 \pm 3.1 \%$ from seven Thermosorb/N cartridges loaded with SS samples and $97.8 \pm 2.8 \%$ from eight cartridges loaded with ETS samples. We also spiked eight filters used for sampling SS from the sidestream apparatus. Recovery from the filters was $96.1 \pm 2.1 \%$. 


\subsubsection{Comparison of Thermosorb/N Cartridge and Impinger Methods of Sample Collection}

In situ artifact formation of $\mathrm{N}$-nitrosamines in the Thermosorb/ $\mathrm{N}$ cartridges has been tested by the manufacturer by passing an air stream containing oxides of nitrogen $\left(\mathrm{NO}_{\mathrm{X}}\right)$ through a cartridge spiked with the respective amine precursors of the $\mathrm{N}$-nitrosamines. This configuration is similar to a real artifact formation situation for outdoor air. The manufacturer did not find any detectable level of N-nitrosamines, and thus, concluded that the cartridges are artifact free. We did not repeat this experiment. However, we did evaluate the cartridges for artifact formation when used for sampling VNA in ETS. This was done by comparing the cartridge method with the validated aqueous solution-impinger method that is traditionally used for sampling tobacco smoke. The test sampling line contained a Thermosorb/N cartridge placed after an open-faced Cambridge filter. The reference sampling line contained three midget-impingers in series, each having $15 \mathrm{~mL}$ of $\mathrm{pH} 4.5$ citrate-phosphate buffer with $20 \mathrm{mM}$ ascorbic acid, placed downstream of an open-faced Cambridge filter. Three commercial cigarettes were smoked in the chamber for each of three trials. ETS samples were collected from the chamber (operated at 0.22 air exchanges per hour) at $2 \mathrm{~L}^{-1}$ min for 250 minutes, with the exception of trial 2 for which the sampling period was 300 minutes. The samples were analyzed as described above. Results of the comparison of the two sampling methods are presented in Table 5.8.

Table 5.8. Comparison of two sampling methods for $\mathbf{N}$-nitrosamines in ETS, ng/cigarette.

\begin{tabular}{|c|c|c|c|c|}
\hline & \multicolumn{2}{|c|}{ NDMA } & \multicolumn{2}{c|}{ NPYR } \\
\hline Trial No. & Cartridge & Impingers & Cartridge & Impingers \\
\hline 1 & 592 & 548 & 135 & 149 \\
\hline 2 & 573 & 563 & 158 & 137 \\
\hline 3 & 578 & 542 & 133 & 150 \\
\hline & & & & 145 \\
\hline Average & 581 & 551 & 142 & 7.4 \\
\hline Std. Dev. & 9.7 & 10.9 & 13.9 & $5 \%$ \\
\hline C. V. ${ }^{2}$ & $2 \%$ & $2 \%$ & $10 \%$ & \\
\hline
\end{tabular}

a. C. V.: Coefficient of Variation $=[$ Std. Dev X 100] $/$ Average.

For NPYR, the average emission factors determined by the two methods do not differ significantly $(p<0.05)$. There was however, a statistically significant difference in the means for NDMA. The difference is probably due not to artifact formation in the cartridge but rather to a slightly lower recovery of NDMA from the impinger solution, as shown in the recovery studies in (Table 5.7). Consequently, we concluded that the Thermosorb/N cartridge would be suitable for sampling VNA in cigarette smoke and would provide emission factors comparable to those obtained from the impinger method. 


\subsection{Sampling and Chemical Analysis for Other Species}

\subsubsection{Aldehydes}

Samples of the low molecular-weight aldehydes (formaldehyde, acetaldehyde, and acrolein) were collected on silica Sep-Pak cartridges (Part No. 37500, Millipore Corp.) impregnated with an acid solution of 2,4-dinitrophenylhydrazine (DNPH) as described by Kuwata et al. (1983). Cartridges were stored in a sealed pouch in a freezer at $-10^{\circ} \mathrm{C}$. A blank sample was collected from the chamber prior to initiation of an experiment. The blank sample was collected for 40 minutes at an air flow rate of 0.88 $\mathrm{L} \mathrm{min}^{-1}$. During an ETS experiment, aldehyde samples were collected at a flow rate of $0.255 \mathrm{~L} \mathrm{~min}^{-1}$ from 0 to 100 minutes elapsed time and from 150 to 250 minutes elapsed time (two sequential samples). A schematic diagram of the sampling train for the collection of aldehydes from the chamber is shown in Figure 5.2. This consisted simply of the Sep-Pak cartridge followed by a mass-flow controller and pump.

Samples of aldehydes and VOCs were collected simultaneously from the SSA as shown schematically in Figure 5.5. A $1500 \mathrm{~cm}^{3} \mathrm{~min}^{-1}$ flow of nitrogen was injected between the SSA and the inline filter to dilute the sample stream. The aldehyde samples were collected downstream of the filter at a flow rate of $30 \mathrm{~cm}^{3} \mathrm{~min}^{-1}$ for 10 to 11 minutes, the time required to smoke a single cigarette in the SSA. The sample flow rate was measured downstream of the peristaltic pump using a bubble flowmeter during and immediately after completion of sampling. In order to avoid overloading the aldehyde sampler, only a fraction $(0.1)$ of the total flow generated by one cigarette was collected for analysis.

The aldehyde samples were analyzed within two weeks of sample collection using the method of Fung and Grosjean (1981). Each sampler was eluted with $2 \mathrm{~mL}$ of glass-distilled acetonitrile and the eluate was made up to volume in a $2-\mathrm{mL}$ volumetric vial. Extracts were diluted 1:1 with distilled water before analysis. The analysis was performed with a high-performance liquid chromatograph (HPLC) equipped with a diode-array ultraviolet detector (Series 1090, Hewlett-Packard Co.). Five-microliter aliquots of the diluted extracts were injected into the instrument using an auto injector. The compounds were separated on a 2.1-mm I.D. x 15-cm long, reverse-phase Nova-Pak C18 column (Waters Chromatography) using an isocratic solvent program with a $60: 30: 10 \mathrm{v} / \mathrm{v} / \mathrm{v}$ mixture of water, acetonitrile and tetrahydrofuran as the mobile phase. The peak height responses for the formaldehyde, acetaldehyde and acrolein hydrazone derivatives were measured at a wavelength of $365 \mathrm{~nm}$. Multi-point, external calibrations were prepared for each experiment by analyzing serial dilutions of the purified aldehyde hydrazone derivatives made up in acetonitrile and water $(1: 1 \mathrm{v} / \mathrm{v})$. 


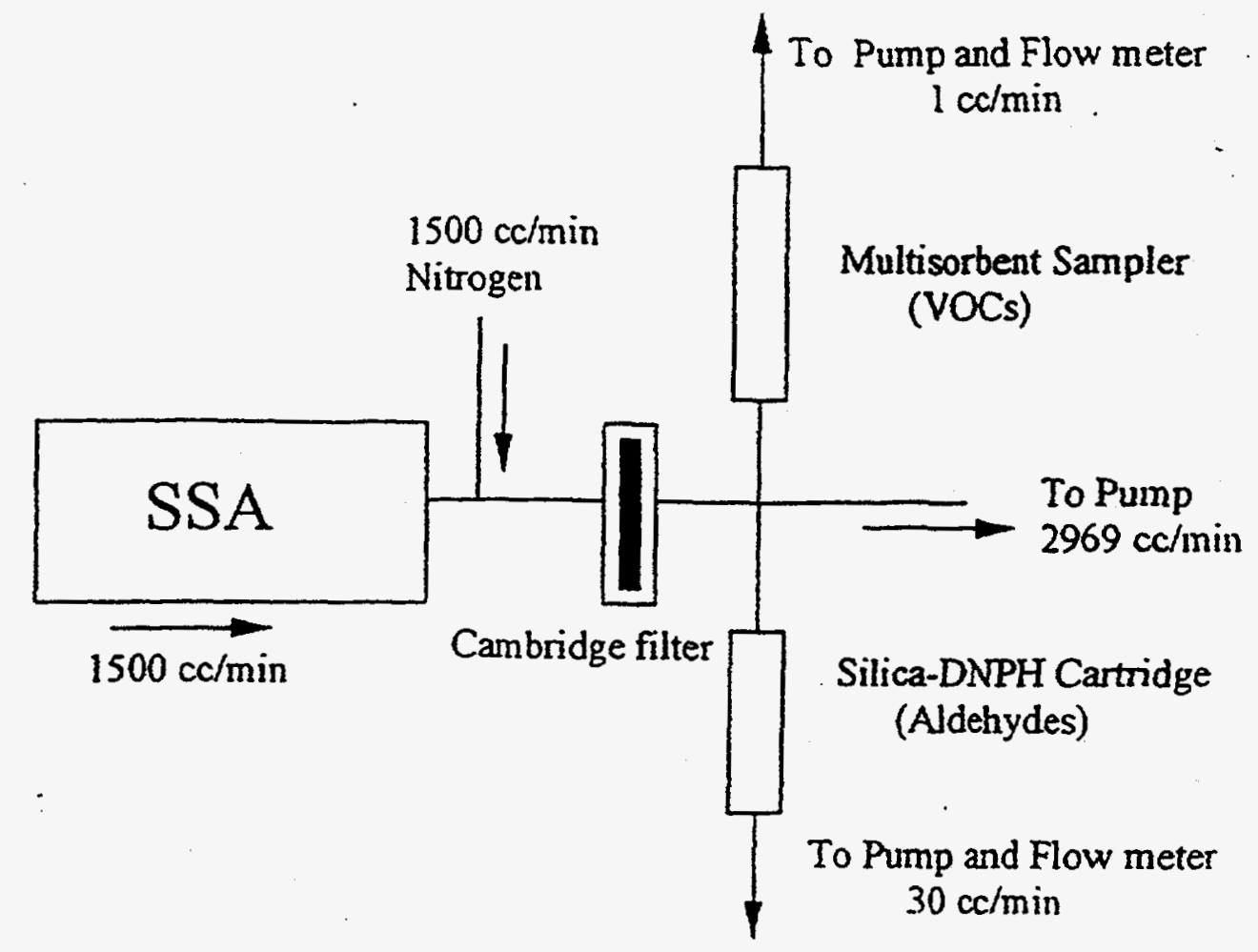

Figure 5.5. Schematic diagram of the sampling trains for aldehydes and VOCs in SS.

\subsubsection{Volatile Organic Compounds}

Samples of VOCs were collected on commercially available multisorbent samplers (Part No. ST032, Envirochem, Inc., Kemblesville, PA) which are packed with glass beads at the inlet followed by Tenax-TA, Ambersorb XE-340 and activated charcoal, in series (Hodgson and Girman, 1989). These multisorbent samplers are reusable. Prior to each use, they were cleaned and conditioned by heating them to $300^{\circ} \mathrm{C}$ for $10 \mathrm{~min}$ with a helium purge flowing in the reverse direction of gas flow during sample collection. The samplers were capped at both ends with Nylon Swagelok caps fitted with Teflon ferrules. The capped samplers were individually sealed in elongated culture tubes.

ETS samples for the quantitative analysis of VOCs were collected beginning at $0,1,2$, and 3 hours elapsed time after initiation of the experiment, i.e., four sequential samples were collected. The sampling rate was about $36.5 \mathrm{~cm}^{3} \mathrm{~min}^{-1}$. Two blank samples were simultaneously collected from the chamber at a flow rate of $200 \mathrm{~cm}^{3} \mathrm{~min}^{-1}$ for 25 minutes before the cigarettes were smoked. The sampling flow rates were 
regulated with electronic mass-flow controllers placed between the samplers and pumps. The samples were stored in a freezer at $-10^{\circ} \mathrm{C}$ and analyzed within two days of collection.

For determination of SS emission factors, a VOC sample was collected from the SSA simultaneously with the aldehyde sample (Figure 5.5). As shown in the figure, the SS sample was diluted with a $1,500 \mathrm{~cm}^{3} \mathrm{~min}^{-1}$ flow of nitrogen. Only a very small sample of VOCs could be used for analysis without overloading the GC/MS system. Consequently, samples were collected at a flow rate of $1 \mathrm{~cm}^{3} \mathrm{~min}^{-1}$ over the 10 to 11 minute period required to smoke one cigarette in the SSA. The sampling flow rate was controlled with a peristaltic pump and measured periodically during this period with a bubble flowmeter. The samples were stored in a freezer at $-10^{\circ} \mathrm{C}$ and analyzed within two days of sample collection.

The analytical procedures for VOCs collected on multisorbent samplers have previously been described (Hodgson and Girman, 1989). In brief, a sample with an added internal standard is thermally desorbed from a sampler, concentrated and introduced into a capillary GC with a UNACON 810A (Envirochem, Inc.) sample concentrating and inletting system. This instrument passes the sample through dual sequential traps to concentrate the sample. Sample components are resolved with a GC (5890 Series II, Hewlett-Packard Co.) equipped with liquid nitrogen sub-ambient cooling and a 30-m x 0.25-mm I.D. $x$ 1.0- $\mu \mathrm{m}$ thick film fused-silica capillary column (Rtx-5, Restek Corp.). The GC is connected via a direct capillary interface to a Series 5970B Mass Selective Detector (MSD) equipped with MS ChemStation software (Hewlett-Packard Co.). The MSD is mass tuned using perfluorotributylamine. It was operated to scan a mass range of $m / z$ 33-300.

Figure 5.6 presents an example of the total-ion-current chromatograms of the VOC samples collected from the environmental chamber. Compounds were tentatively identified by comparing the unknown spectra with spectra contained in the EPANIH Mass Spectral Data Base (Heller and Milne, 1978). Identifications were confirmed by analyzing authentic standards of the compounds under identical conditions.

For the quantitative analysis of each compound of interest, a mass ion with a high relative abundance was chosen as the quantitative ion, and a characteristic ion was chosen as a qualifying ion for confirmation of compound identity. These target mass ions were extracted from the total-ion-current chromatograms and their peak areas were integrated using the software. 


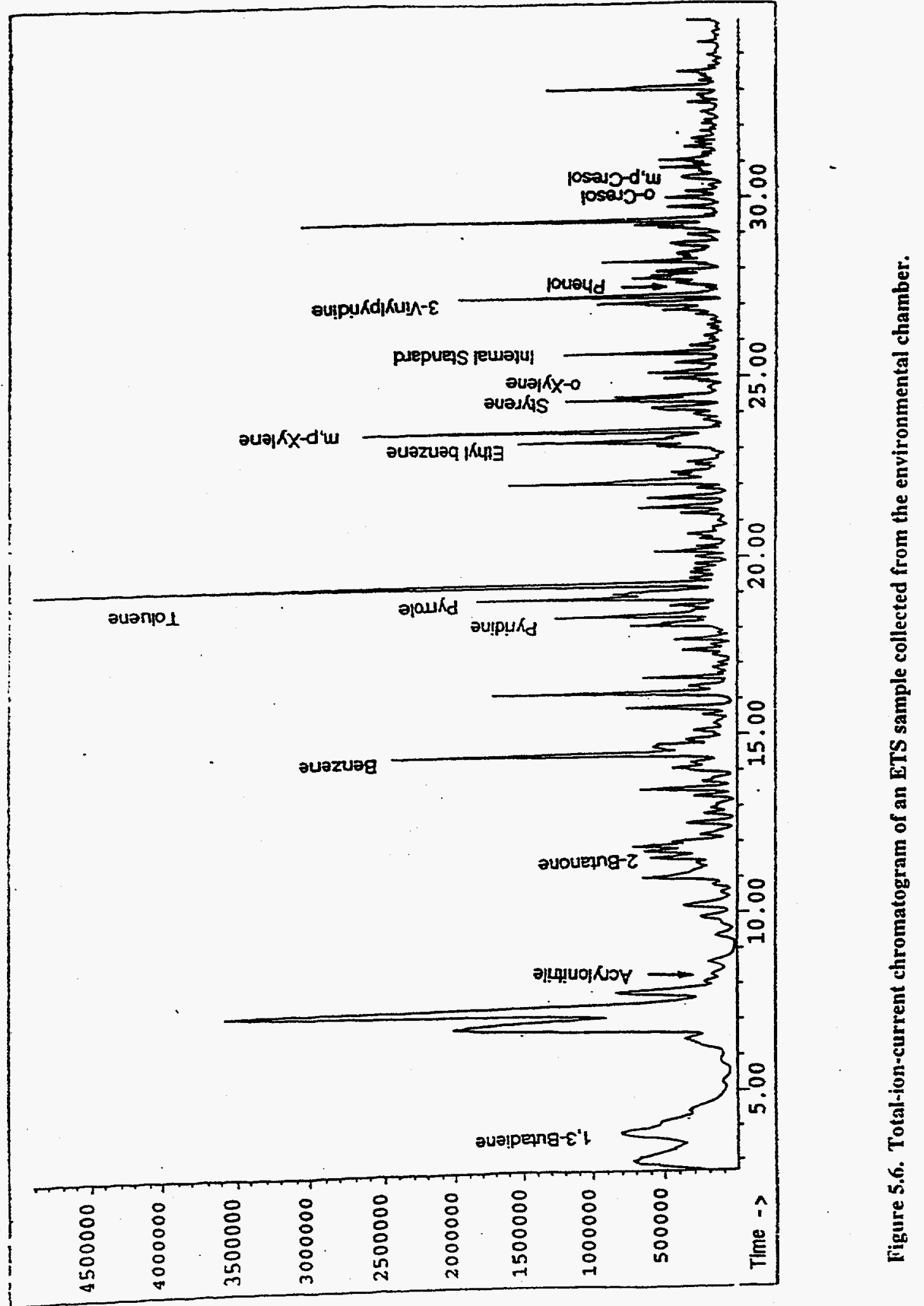


Authentic standards used for compound identifications and calibrations were obtained from commercial sources (e.g., Aldrich Chemical, Co.). Standard gas mixtures of the more volatile VOCs were prepared by injecting a several-microliter aliquot of a liquid mixture of the compounds of interest into a 2-L flask with septum cap which was heated and maintained at $65^{\circ} \mathrm{C}$ (Riggin, 1984). A sample was withdrawn from the flask with a gas-tight syringe and injected into a helium gas stream flowing through a conditioned multisorbent sampler in the direction of sample gas flow. The internal standard was introduced onto the sampler at this time. The sampler was then analyzed using the same procedure as for the samples. Multipoint internal-standard calibrations were prepared by analyzing a range of volumes of the gas mixture. Fresh standard gas mixtures were prepared on each day of analysis.

Standard solutions of the less volatile compounds were prepared in a highly volatile solvent such as methanol. Microliter quantities of these solutions were injected onto the inlets of all-Tenax samplers. Then, the spiked samplers were purged with helium to remove most of the solvent. The internal standard was then added to each sampler. Multi-point internal-standard calibrations were prepared by the analysis of samplers spiked with a range of masses.

The internal standard consisting of approximately $80 \mathrm{ng}$ of 1-bromo-4-fluorobenzene was added to all samplers, including standards, immediately prior to analysis. The internal standard was generated by a gravimetrically-calibrated diffusion source. It was transferred from the source with a gas-tight syringe and introduced into the helium gas stream flowing through a sampler in the direction of sample gas flow.

Complete multi-point calibrations for the compounds of interest were prepared prior to the measurement of both the SS and the ETS samples. Additional calibrations were performed periodically throughout the analysis of each type of sample. For the ETS samples, several complete multi-point calibrations were performed for all compounds.

\subsubsection{Nicotine}

Nicotine in ETS is almost wholly in the vapor phase (Eatough, et al., 1986; Hammond, et al., 1987). Sorbent tubes containing 20/40 mesh XAD-4 (SKC West, Inc.) were used to collect nicotine from both SS and ETS samples. The sorbent tube consists of two sections. The front section contains $80 \mathrm{mg}$ of resin, and the back-up section contains $40 \mathrm{mg}$ of resin. A sealed tube was cracked open just before sampling, and the sample (SS or ETS) was drawn through the tube using a vacuum pump. The desired flow rate was maintained using a mass-flow controller. In ETS, a single nicotine sample was collected from the chamber starting at time zero at a flow rate of $2,000 \mathrm{~cm}^{3} \mathrm{~min}^{-1}$ for 250 minutes. A schematic of the sampling train for nicotine in ETS is shown in Figure 5.7a. 
For nicotine in SS, a sample of vapor-phase nicotine was collected from the SSA upstream of the in-line filter at a sampling rate of $20 \mathrm{~cm}^{3} \mathrm{~min}^{-1}$. The remainder (and majority) of the generated SS was drawn through a Teflon-coated glass-fiber filter at $1480 \mathrm{~cm}^{3} \mathrm{~min}^{-1}$, as shown in Figure $5.7 \mathrm{~b}$. In addition to the nicotine collected on the sorbent tube, nicotine that had condensed on the interior of the SSA was recovered and analyzed.

The sample-loaded sorbent tube was capped and stored in a freezer. For analysis, the XAD-4 resin from both sections of the sorbent tube was emptied into a $2-\mathrm{mL}$ volumetric vial. Ethyl acetate (EA) was modified by addition of $0.01 \% \mathrm{v} / \mathrm{v}$ triethylamine (TEA) to prevent any adsorption of nicotine on glass surfaces (Ogden, et al., 1989). The interior of the sorbent tube was rinsed with $2 \mathrm{~mL}$ of the modified EA solution which was then added to the XAD-4 resin. The condensate was recovered by rinsing the apparatus with the modified EA solution and 4-5 $\mathrm{mL}$ of methanol. This rinse was filtered to remove cigarette ash, and the final volume was adjusted to $25 \mathrm{~mL}$ using the EA solution.

Nicotine was analyzed in the extracts of the sorbent tube and the SSA condensate. An aliquot of each extract was injected into a gas chromatograph (Model GC-9A, Shimadzu Corp.) equipped with a DBWAX capillary column ( $30 \mathrm{~m} \times 0.32 \mathrm{~mm}$ I.D. $\times 0.25 \mu \mathrm{m}$ thick film fused-silica, J. \& W. Scientific, Inc.) and a nitrogen-phosphorous detector (DET, Walnut Creek, CA). The peak areas of the chromatograms were integrated using a Shimadzu C-R3A integrator.

Quinoline has often been used as an internal standard for nicotine measurements. However; we found detectable levels of quinoline in the SS samples (both in the XAD-4 resin extracts and the SSA condensates). Therefore, we did not use the internal standard method of quantitation. Instead, nicotine was quantified in all samples using an external calibration curve.

\subsubsection{Airborne Particulate Matter}

Emissions of particulate matter (PM-2.5 equivalent) in ETS were determined by collecting particle-samples on an open-faced Teflon-filter (Teflo, Gelman Sciences) using a vacuum pump. The filter was followed by the Thermosorb/N cartridge used to collect the $\mathrm{N}$-nitrosamine sample (Figure 5.2). For each ETS experiment, a single sample was collected at a flow rate of $4 \mathrm{~L} \mathrm{~min}^{-1}$ for 250 minutes starting at time zero. A cyclone was not used as a pre-filter to remove particles larger than $3.5 \mu \mathrm{m}\left(\mathrm{D}_{50}\right)$ because there were concerns about possible losses of vapor-phase VNA to the surface of the cyclone as the air stream moved through the sampling train. In addition, experimental work at LBL (Offermann, et al., 1985; Xu, et al., 1994) has demonstrated that the mass median particle diameter of ETS is about $0.2 \mu \mathrm{m}$, with a $\sigma_{\mathrm{g}}$ of about 2. Based on these studies, $98 \%$ of the particles collected in the chamber would be less than $0.8 \mu \mathrm{m}$. Thus, the samples were equivalent to those which would have been measured with a PM-2.5 sampler with a 
cyclone pre-filter, i.e., they are equivalent to a PM-2.5 sample. Sample mass was determined by weighing the filters before and after sample collection on a Cahn Electrobalance.

The SS particulate matter sample was collected concurrently with the nicotine sample as shown in Figure 5.7b. A Teflon-coated glass-fiber filter was used. These filters will also collect some condensed water vapor. The mass of particulate matter on a filter was determined as the difference in the weight of the filter before and after sampling. No attempt was made to correct for the water vapor. In addition, particulate matter plus any other materials that had condensed onto the inner walls of the SSA were recovered by rinsing the apparatus with solvent. The extract was filtered to remove ash particles, and

a.
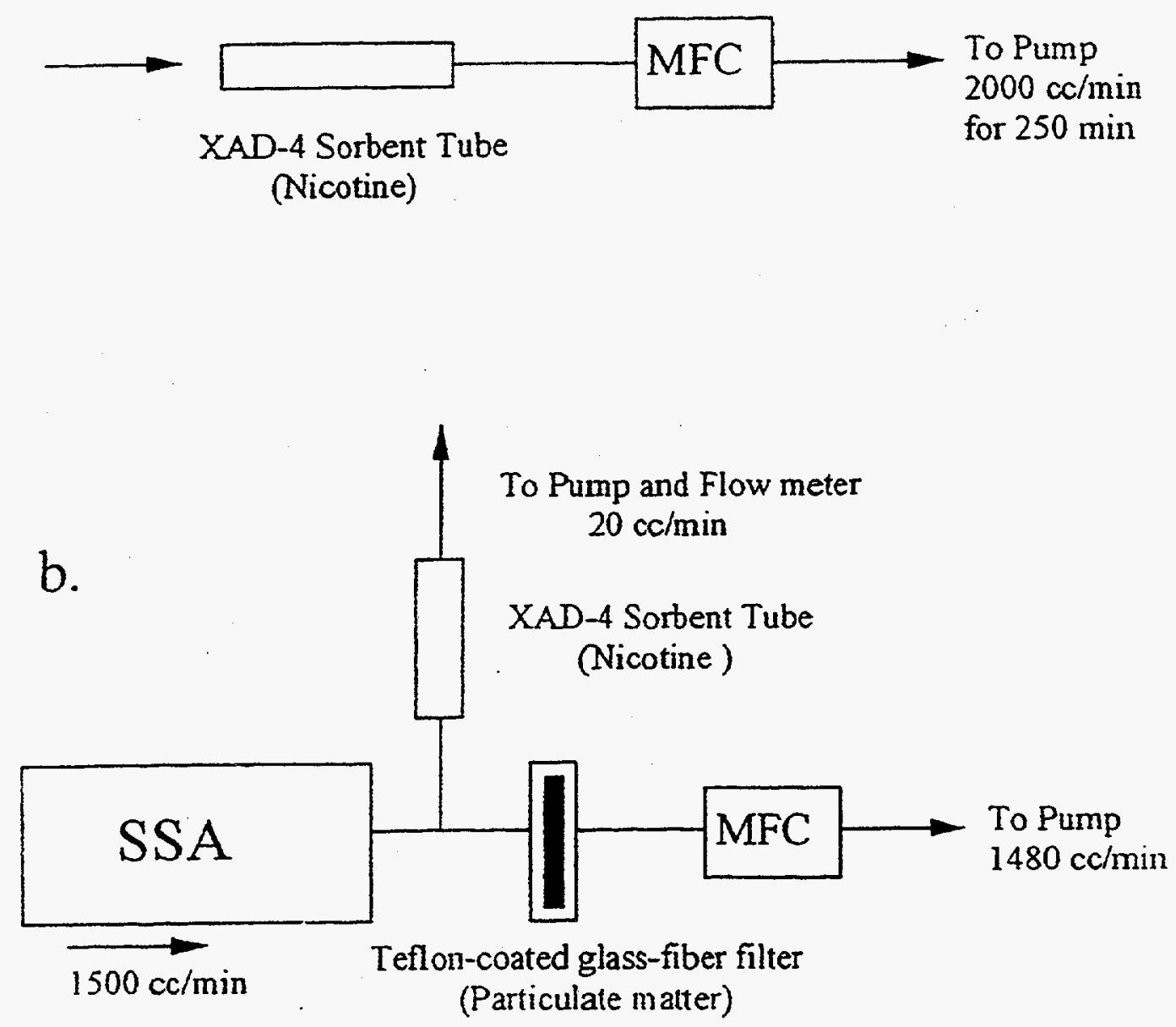

Figure 5.7. Schematic diagram of the sampling train for collection of a.) nicotine in ETS and b.) nicotine and particulate matter in SS. 
the mass of the condensate was weighed after evaporating the solvent. The total SS particulate mass was defined as the mass collected onto the filter plus the mass collected from the SSA.

There are substantial artifact problems associated with measuring emission factors for SS particulate matter since the measurement is highly dependent upon the sampling conditions. The hot and highly concentrated mixture of gases and particles that is emitted from the burning cone of the cigarette cools rapidly and some of the vapors (i.e., water and organic compounds) condense on the filter and the inside surfaces of the SSA. Although the condensate collected on the filter and SSA can be recovered and reproducibly measured, a different SS emission factor would be reported if a different apparatus with a different dilution factor and surface-to-volume ratio was used. The relevance of such SS particulate emission factors to ETS particle emission factors, which are produced under conditions of rapid and very high dilution and cooling, is not clear. Nevertheless, the results of this measurement have been presented for comparison to other reported SS emission factors.

\subsection{5 $\mathrm{NO}_{\mathrm{x}}$ and $\mathrm{CO}$}

The $\mathrm{NO}_{\mathrm{x}}$ and $\mathrm{NO}$ measurements were made with a Model 14 Chemiluminescent $\mathrm{NO}-\mathrm{NO}_{\mathrm{x}}$ gas analyzer (Thermo Electron Corp.). With this instrument, concentrations of $\mathrm{NO}_{2}$ are determined as the difference between $\mathrm{NO}_{x}$ and NO. Standard gas cylinders of $\mathrm{NO}$ (960 ppm in argon) and $\mathrm{NO}_{2}$ (496 ppm in nitrogen) were further diluted in air to prepare calibration curves. For the analysis of $\mathrm{NO}_{\mathrm{x}}$ in ETS, air was drawn continuously from the middle of the chamber at a height of $1.2 \mathrm{~m}$. The sampling line was 3-mm I.D. stainless-steel tubing. The $\mathrm{NO}_{x}$ analyzer was operated continuously beginning the night before the experiment. $\mathrm{NO}_{\mathrm{x}}$ was not measured in the SS experiments.

The $\mathrm{CO}$ levels in the chamber were monitored once per minute using a $\mathrm{CO}$ diffusion sampler based on electrochemical detection (Drager, Model 190). There is no active pumping with this device, rather the $\mathrm{CO}$ in air diffuses to the surface of the detector and is measured electrochemically. A data logger was used to record all of these measurements. The $\mathrm{CO}$ data were examined to determine if there had been any significant changes in concentration over the period of each chamber experiment which would indicate unexpected air leakage into the chamber and a larger air exchange rate than determined from the $\mathrm{SF}_{6}$ measurements. The average $\mathrm{CO}$ concentration in the chamber was calculated from the one-minute readings. The CO sampler was calibrated with 0 and $60 \mathrm{ppm}$ calibration gases prior to the each experiment. CO was not measured in the SS experiments. 


\subsection{Data Reduction and Analysis}

\subsubsection{Emission Factors for ETS}

Chamber concentrations of all of the analytes of interest were predicted to quickly reach a maximum soon after the introduction of the cigarette smoke and then decay exponentially, but very slowly, over the next 4 hours of the experiment. The measured average concentration of any species, $C_{m}$, over its sampling period can be expressed as:

$$
C_{m}=\frac{1}{\Delta t} \int_{t_{i}}^{t_{f}} C(t) d t,
$$

where $C(t)$ is the concentration in the chamber at any time, $t ; \Delta t=\left(t_{f}-t_{i}\right)$ is the sampling interval for which $t_{i}$ and $t_{\mathrm{f}}$ are the initial and final sampling times, respectively.

The concentration in the chamber will decay exponentially over time due to the small rate of infiltration and the removal of air by sampling:

$$
C(t)=C(0) e^{-a t},
$$

where $C(0)$ is the concentration in the chamber at the start of the experiment (i.e., immediately after cigarette smoking is completed and the chamber has been uniformly mixed) and $a$ is a constant that accounts for losses due to infiltration and the removal of air by sampling. The integral of $\mathrm{C}(\mathrm{t})$ over a sampling interval is equivalent to $C_{m}$. Therefore,

$$
C_{m}=\frac{1}{\Delta t} \int_{t_{i}}^{t_{f}} C(0) e^{-a t} d t
$$

Integrating equation (4) between $t_{i}$ and $t_{f}$ gives:

$$
C_{m}=\frac{C(0)}{a \Delta t}\left(e^{-a t} i-e^{-a t} f\right) .
$$

The concentration in the chamber at the initiation of sampling is,

$$
C(0)=\frac{N E}{V},
$$


where $\mathrm{N}$ is the number of cigarettes smoked, $\mathrm{E}$ is the emission factor in $\mu \mathrm{g} /$ cigarette, and $\mathrm{V}$ is the volume of the chamber in $\mathrm{m}^{3}$. It is assumed that any losses during the smoking period would be very small since the introduction and mixing of ETS was completed in 30 minutes or less, and the infiltration rate was negligible. Substituting equation (6) into equation (5) gives

$$
C_{m}=\frac{N E}{V a \Delta t}\left(e^{-a t} i-e^{-a t} f\right)
$$

Since $C_{m}, N, V$ and a are known for each experiment and species, equation (7) can be solved for the value of $E$ for each analyte of interest.

There is also some possibility of losses due to chemical reactions or to deposition to the surfaces of the chamber. For the VOCs which were collected with a multisorbent sampler, samples were collected starting at elapsed times of $0,1,2$, and 3 hours. For most of the VOCs, there was no evidence of changes in concentration as a function of time, other than what was predicted by the air exchange rate. This indicated that there were minimal losses due to chemical reactions or to deposition. However, for several of the VOCs there was evidence of additional losses which we presumed to be due to surface deposition. Emission factors for these compounds were calculated so as to take these losses into account. Losses of the other vapor-phase compounds which were measured only as an average over the period of the experiment (e.g., N-nitrosamines) may have occurred but can not be determined. Since losses to the stainless-steel chamber walls are likely to be smaller than would occur in buildings, the measured emission factors for these compounds constitute an upper bound for exposure estimates.

Equation (7) was modified to account for removal by means other than air infiltration, e.g., deposition onto the chamber surfaces or chemical reactions, by introducing a new term, $k$. Equation (8) assumes that all VOC removal processes follow a first order exponential decay.

$$
C_{m}=\frac{N E\left\{e^{-(a+k) t_{f}}-e^{-(a+k) t_{i}}\right\}}{\left[V(a+k)\left(t_{f}-t_{i}\right)\right]} .
$$

Small depositional losses of ETS particles to surfaces have been shown to occur during experiments by Offermann, et al. (1985) in a room in a test house. These investigators estimated a loss rate constant, $k$, of about $0.1 \mathrm{~h}^{-1}$. For the stainless-steel chamber, the $k$ value is expected to be lower. However, there are no experimental data. As a first-order estimate, we selected a $k$ value of $0.05 \mathrm{~h}^{-1}$ to correct for the deposition losses of particles in the chamber surfaces. 
Emission factors for the analytes were expressed as mass per cigarette as well as mass per mg of tobacco consumed. Mass per cigarette is the mass of analyte generated by smoking a cigarette to the standard length. Mass per $\mathrm{mg}$ of tobacco consumed is the mass of the analyte generated from one milligram of tobacco. Mass of tobacco consumed was calculated from the mass of tobacco per unit length of cigarette and the smoked length.

\subsubsection{Emission Factors for SS}

Emission factors for VOCs and aldehydes in the SS experiments were calculated as:

$$
E=\frac{m}{f N}
$$

where $E$ is the emission rate in $\mu \mathrm{g} / \mathrm{cig}$; $\mathrm{m}$ is the mass of analyte collected on the sampler in $\mu \mathrm{g}$; $\mathrm{f}$ is the fraction of SS sampled; and $\mathrm{N}$ is the number of cigarettes smoked ( $\mathrm{N}=1$ for all of the experiments). The fraction sampled, $f$, is:

$$
f=\frac{Q_{S}}{Q_{t}}
$$

where $Q_{S}$ is the sampling flow rate in $\mathrm{cm}^{3} \mathrm{~min}^{-1}$, and $Q_{t}$ is the total flow rate through the SSA in $\mathrm{cm}^{3}$ $\min ^{-1}$.

Nicotine and particulate matter were condensed in the SSA. Therefore, the SS emission factors for these constituents were calculated as:

$$
E=\frac{m}{f N}+\frac{m_{s}}{N},
$$

where $\mathrm{m}_{\mathrm{s}}$ is the mass condensed in the SSA in $\mu \mathrm{g}$.

For nitrosamines, the sampler, the SSA condensate and the filter were analyzed. Therefore, the SS emission factors for nitrosamines were calculated as:

$$
E=\frac{m}{f N}+\frac{m_{s}}{N}+\frac{m_{f}}{N}
$$

where $m_{f}$ is the mass condensed on the filter in $\mu \mathrm{g}$. 


\subsubsection{Experimental Uncertainties in the Measurements}

For the $\mathrm{N}$-nitrosamine, aldehyde, VOC, and nicotine measurements, the experimental uncertainties associated with sampling and analysis cannot be estimated from the standard deviations of the means for replicate measurements because there were insufficient numbers of replicate samples. Consequently, uncertainties in the ETS emission factors due to sampling and analysis were estimated using propagation of errors. This method estimates the uncertainties in a dependent variable (e.g., the emission factor reported as $\mu \mathrm{g}$ per cigarette) from the propagation of the uncertainties of the individual variables that are used to calculate that dependent variable. The method assumes that the errors are random and independent for each of the contributing variables (Shoemaker, et al., 1974).

Uncertainties in the values of $E$ (mass per cigarette) $(\Delta E)$ were estimated based on equation (7), which in rearranged form is:

$$
E(\mu g / c i g)=\frac{C_{m} \text { Va } \Delta t}{\left[N\left(e^{-a t_{i}}-e^{-a t_{f}}\right)\right]} .
$$

Values of $C_{m}$ from the chamber experiments were calculated from the chromatographic peak areas or heights of the target compounds and the volumes of air sampled from the chamber, i.e.,

$$
\mathrm{C}_{\mathrm{m}}=\frac{\mathrm{m}_{\mathrm{T}}}{\mathrm{V}_{\mathrm{air}}}
$$

where $\mathrm{m}_{\mathrm{T}}$ is the mass of the target compound collected from the chamber, and $\mathrm{V}_{\mathrm{air}}$ is the volume of air sampled. Then,

$$
\mathrm{m}_{\mathrm{T}}=\frac{\mathrm{m}_{\mathrm{inj}} \mathrm{V}_{\text {soln }}}{\mathrm{v}_{\text {inj }}}
$$

where $\mathrm{m}_{\mathrm{inj}}$ is the mass of the target compound in the sub-sample injected into the chromatograph and $\mathrm{V}_{\text {soin }} / \mathrm{V}_{\mathrm{inj}}$ is the ratio of the total volume of the sample solution to the volume of the injected sample. The value of $m_{i n j}$ is calculated from $A_{T}$, the chromatographic peak area (or height) of the target compound in the chromatogram, and $\mathrm{RF}_{\mathrm{T}}$ is the response factor for the target compound based on the slope of the calibration curve, so that,

$$
m_{T}=\frac{A_{T} V_{\text {soln }}}{R F_{T} V_{\text {inj }}}
$$


Combining equations (14) and (16) with equation (13) yields:

$$
E(u g / c i g)=\frac{A_{T} V_{\text {soln }} V a \Delta t}{R F_{T} V_{\text {inj }} N V_{\text {air }}\left(e^{-a t_{i}}-e^{-a t} f\right)}
$$

Because the uncertainties in $\mathrm{E}$ will be dominated by the terms with the greatest uncertainties, the uncertainties in some terms in equation (17) can be neglected (Shoemaker, 1974). Specifically, the uncertainties in $\mathrm{V}, \Delta t, t_{i}$ and $t_{f}$ are all negligible (i.e., less than $1 \%$ ). The value of $N$ is a constant ( 3 cigarettes) and therefore does not have any uncertainty. $V_{a i r}$ was measured with a mass flowmeter and a stop watch and has an uncertainty of about $2 \%$. The relative variance in $E, \Delta E / E$, can then be expressed in terms of the relative variances of the remaining terms:

$$
\begin{gathered}
(\Delta \mathrm{E} / \mathrm{E})^{2}=\left(\Delta \mathrm{A}_{\mathrm{T}} / \mathrm{A}_{\mathrm{T}}\right)^{2}+\left(\Delta \mathrm{RF}_{\mathrm{T}} / \mathrm{RF}_{\mathrm{T}}\right)^{2}+\left(\Delta \mathrm{V}_{\mathrm{soln}} / \mathrm{V}_{\mathrm{soln}}\right)^{2}+\left(\Delta \mathrm{V}_{\mathrm{inj}} / \mathrm{V}_{\mathrm{inj}}\right)^{2}+ \\
(\Delta \mathrm{a} / \mathrm{a})^{2}+\left(\Delta \mathrm{V}_{\mathrm{air}} / \mathrm{V}_{\mathrm{air}}\right)^{2}+\left[\Delta\left(\mathrm{e}^{-\mathrm{at}_{\mathrm{i}}}-\mathrm{e}^{-\mathrm{atf}_{\mathrm{f}}}\right) /\left(\mathrm{e}^{\mathrm{at}_{\mathrm{i}}}-\mathrm{e}^{-\mathrm{at}}\right)\right]^{2} .
\end{gathered}
$$

The values of the relative uncertainties in the response factors, $\Delta R F_{T} / R F_{T}$, were estimated from the variability in the peak height or area measurements of the calibration standards. These are summarized in Table 5.9. The relative uncertainty in $\Delta \mathrm{A}_{\mathrm{T}} / \mathrm{A}_{\mathrm{T}}$, based on replicate analyses, is about 0.01 (1\%); the relative uncertainties in $V_{\text {soln }}$ and $V_{\text {inj }}$, based on the manufacturers' specifications for the volumetric flasks and injection syringes, are each about $0.01(1 \%)$; the relative uncertainty of the air exchange rate, a, was estimated by propagating the uncertainties for chamber infiltration and sampling-induced infiltration. This value was about $0.03(3 \%)$. The relative uncertainty in the last term in equation (17) was then calculated to be less than $0.01(<1 \%)$. Based on these estimates, the relative uncertainties in the ETS emission factors for the measured compounds were calculated and these are presented in Table 5.10 as percentages $(\Delta \mathrm{E} / \mathrm{E}) \mathrm{x}$ $100 \%$.

In general, the relative uncertainties due to the variability in sampling and analysis were about 10 to $15 \%$, with the exception of 1,3-butadiene, which was about $25 \%$. Variations in ETS emission factors among brands of cigarettes that are not significantly greater than these sampling and analysis uncertainties should not be considered to be significantly different. 
Table 5.9. Relative uncertainties in the analytical response factors for the measured compounds.

\begin{tabular}{|l|c|l|c|}
\hline Compound & $\left(\Delta \mathbf{R F}_{\mathbf{T}} / \mathbf{R F}_{\mathbf{T}}\right) \mathbf{x} \mathbf{1 0 0 \%}$ & Compound & $\left(\Delta \mathbf{R F}_{\mathbf{T}} / \mathbf{R F}_{\mathbf{T}}\right) \mathbf{1 0 0 \%}$ \\
\hline Acetaldehyde & 13 & Formaldehyde & 9.0 \\
\hline Acrolein & 13 & 3-Methyl-1-butanol & 16 \\
\hline Acrylonitrile & 15 & Nicotine & 8 \\
\hline Benzene & 5.6 & N-Nitrosodimethylamine & 12 \\
\hline 1,3-Butadiene & 25 & N-Nitrosopyrrolidine & 12 \\
\hline 2-Butanone & 6.7 & Phenol & 8.1 \\
\hline Butyraldehyde & 6.2 & Pyridine & 18 \\
\hline Butyl acetate & 6.1 & Pyrrole & 15 \\
\hline o-Cresol & 13 & Styrene & 14 \\
\hline m,p-Cresol & 14 & Toluene & 4.4 \\
\hline Ethyl acetate & 6.8 & 3-Vinylpyridine & 8.8 \\
\hline Ethyl acrylate & 13 & m, p-Xylene & 6.2 \\
\hline Ethylbenzene & 6.2 & o-Xylene & 3.3 \\
\hline
\end{tabular}

The uncertainties in the ETS particulate matter emissions, E (PM-2.5), were also estimated by propagation of errors. The emission rate of particulate matter was determined as:

$$
E(P M-2.5)=\frac{\left(M_{L}-M_{0}\right) V}{V_{\text {air }} N}
$$

where $M_{L}$ is the mass of the particle-loaded filter, $M_{0}$ is the mass of the unloaded filter, and $V_{\text {air }}$ is the volume of air drawn through the filter. The estimated relative uncertainty in E (PM-2.5) is then,

$$
[\Delta \mathrm{E}(\mathrm{PM}-2.5) / \mathrm{E}(\mathrm{PM}-2.5)]^{2}=\left[\Delta\left(\mathrm{M}_{\mathrm{L}}-\mathrm{M}_{0}\right) /\left(\mathrm{M}_{\mathrm{L}}-\mathrm{M}_{0}\right)\right]^{2}+\left[\Delta \mathrm{V}_{\text {air }} / \mathrm{N}_{\text {air }}\right]^{2}
$$

ignoring the uncertainties for $\mathrm{V}$ and $\mathrm{N}$ which are constant. The relative uncertainties of PM-2.5 for ETS were estimated to be $2.4 \%$, or less.

The relative uncertainties in the SS emission factors for aldehydes and VOCs were calculated using equation (21).

$$
\begin{gathered}
(\Delta \mathrm{E} / \mathrm{E})^{2}=\left(\Delta \mathrm{A}_{\mathrm{T}} / \mathrm{A}_{\mathrm{T}}\right)^{2}+\left(\Delta \mathrm{RF}_{\mathrm{T}} / \mathrm{RF}_{\mathrm{T}}\right)^{2}+\left(\Delta \mathrm{V}_{\mathrm{soln}} / \mathrm{V}_{\mathrm{soln}}\right)^{2}+\left(\Delta \mathrm{V}_{\mathrm{inj}} / \mathrm{V}_{\mathrm{inj}}\right)^{2}+ \\
\left(\Delta \mathrm{Q}_{\mathrm{S}} / \mathrm{Q}_{\mathrm{S}}\right)^{2}+\left(\Delta \mathrm{Q}_{\mathrm{t}} / \mathrm{Q}_{\mathrm{t}}\right)^{2}
\end{gathered}
$$


Table 5.10. Estimated relative uncertainties in ETS emission factors for the measured compounds, $\mu \mathrm{g} /$ cigarette.

\begin{tabular}{|l|c|l|c|}
\hline Compound & $(\Delta \mathbf{E} / \mathbf{E}) \times \mathbf{1 0 0} \%$ & Compound & $(\Delta \mathbf{E} / \mathbf{E}) \mathbf{1 0 0} \%$ \\
\hline Acetaldehyde & 14 & Formaldehyde & 10 \\
\hline Acrolein & 14 & 3-Methyl-1-butanol & 17 \\
\hline Acrylonitrile & 16 & Nicotine & 8.9 \\
\hline Benzene & 6.8 & N-Nitrosodimethylamine & 13 \\
\hline 1,3-Butadiene & 25 & N-Nitrosopyrrolidine & 13 \\
\hline 2-Butanone & 7.7 & Phenol & 9.0 \\
\hline Butyraldehyde & 7.3 & Pyridine & 18 \\
\hline Butyl acetate & 7.2 & Pyrrole & 16 \\
\hline o-Cresol & 14 & Styrene & 15 \\
\hline m,p-Cresol & 15 & Toluene & 5.9 \\
\hline Ethyl acetate & 7.8 & 3-Vinylpyridine & 9.6 \\
\hline Ethyl acrylate & 14 & m, p-Xylene & 7.3 \\
\hline Ethylbenzene & 7.3 & o-Xylene & 5.1 \\
\hline
\end{tabular}

Table 5.11. Estimated relative uncertainties in SS emission factors for the measured compounds, $\mu \mathrm{g} /$ cigarette.

\begin{tabular}{|l|c|l|c|}
\hline Compound & $(\Delta \mathrm{E} / \mathrm{E}) \times \mathbf{1 0 0} \%$ & Compound & $(\Delta \mathrm{E} / \mathbf{E}) \mathbf{x} \mathbf{1 0 0} \%$ \\
\hline Acetaldehyde & 14 & Formaldehyde & 9.8 \\
\hline Acrolein & 14 & 3-Methyl-1-butanol & 19 \\
\hline Acrylonitrile & 18 & Nicotine & 12 \\
\hline Benzene & 12 & N-Nitrosodimethylamine & 21 \\
\hline 1,3-Butadiene & 27 & N-Nitrosopyrrolidine & 21 \\
\hline 2-Butanone & 13 & Phenol & 13 \\
\hline Butyraldehyde & 12 & Pyridine & 21 \\
\hline Butyl acetate & 12 & Pyrrole & 18 \\
\hline o-Cresol & 17 & Styrene & 18 \\
\hline m,p-Cresol & 18 & Toluene & 11 \\
\hline Ethyl acetate & 13 & 3-Vinylpyridine & 14 \\
\hline Ethyl acrylate & 17 & m, p-Xylene & 11 \\
\hline Ethylbenzene & 12 & o-Xylene & \\
\hline
\end{tabular}


The values in the relative uncertainties in the flow rates, $Q_{s}$ and $Q_{t}$, are approximately $0.02(2 \%)$. For VOCs only, the relative uncertainty of $Q_{s}$ is about 0.1 (10\%). The emission factor for nicotine was calculated based on the combined results for the analysis of the sorbent tube and the SSA extract, and the $\mathrm{N}$-nitrosamine emission factors were calculated by combining the results for the cartridge, the SSA extract and the filter extract. For the extracts, there are no flow rate terms in the uncertainty calculations. The uncertainties for these compounds were estimated by combining the uncertainties of each separate analysis using root-sum-square addition. The estimated uncertainties for the SS emission factors are shown in Table 5.11 .

The relative uncertainty for SS particulate matter is the same as for ETS.

\subsubsection{Lower Limits of Detection}

Lower limits of detection were estimated for all of the compounds measured in ETS and SS (Table 5.12). These values were estimated from the cigarette data, the sample and injection volumes, and the sensitivities of the analytical instruments to the compounds versus the instrumental noise levels. The instrumental limit of detection (ng/injection) was defined as the mass that produced a signal that was three times the height or area of the noise level. For VOCs, the entire sample was injected and analyzed. Therefore, the limits of detection for VOCs in $\mu \mathrm{g} / \mathrm{cig}$ were calculated as the $\mu \mathrm{g}$ per sample values divided by the fractions of tobacco smoke sampled per cigarette. For aldehydes, $\mathrm{N}$-nitrosamines and nicotine, only aliquots of the samples were analyzed; therefore, the ng/injection values were first divided by the fractions of the sample extracts that were analyzed to obtain the $\mu \mathrm{g}$ per sample values. The limits of detection in $\mathrm{ng} / \mathrm{mg}$ were obtained from the mass per cigarette limits of detection by dividing them by the average masses of tobacco smoked per cigarette. The lower limits of detection for the ETS and SS measurements that are shown in Table 5.12 were calculated for the first replicate experiment with cigarette $\mathrm{A}$ as this cigarette and the experimental parameters were reasonably typical.

Seven of the compounds were detected in the chamber background. These were benzene, 2butanone, formaldehyde, styrene, toluene, m,p-xylene, and o-xylene. The experimental chamber concentrations for all seven compounds were well above the background concentrations and, therefore, were easily detectable. 
Table 5.12. Estimated lower limits of detection for the compounds measured in environmental tobacco smoke and sidestream smoke.

\begin{tabular}{|c|c|c|c|c|c|}
\hline & & ETS & ETS & SS & SS \\
\hline Compound & ng/inj & ng/mg & $\mu \mathrm{g} / \mathrm{cig}$ & ng/mg & $\mu \mathrm{g} / \mathrm{cig}$ \\
\hline Acetaldehyde & 0.088 & 39 & 24 & 1.2 & 0.8 \\
\hline Acrolein & 0.12 & 53 & 32 & 1.7 & 1.1 \\
\hline Acrylonitrile & 7 & 42 & 26 & 31 & 21 \\
\hline Benzene & $<1$ & 3 & 2 & 2 & 1 \\
\hline 1,3-Butadiene & 18 & 116 & 71 & 87 & 58 \\
\hline 2-Butanone & 2 & 9 & 6 & 7 & 5 \\
\hline Butyl acetate & 1 & 4 & 3 & 3 & 2 \\
\hline Butyraldehyde & 5 & 29 & 18 & 22 & 14 \\
\hline m,p-Cresol & 6 & 35 & 22 & 27 & 18 \\
\hline o-Cresol & 6 & 35 & 22 & 27 & 18 \\
\hline Ethyl acetate & 1 & 6 & 4 & 5 & 3 \\
\hline Ethyl acrylate & 1 & 5 & 3 & 4 & 3 \\
\hline Ethylbenzene & 1 & 5 & 3 & 4 & 3 \\
\hline Formaldehyde & 0.045 & 20 & 12 & 0.6 & 0.4 \\
\hline 3-Methyl-1-butanol & 4 & 23 & 14 & 17 & 11 \\
\hline Nicotine & 0.41 & 23 & 14 & 108 & 72 \\
\hline N-Nitrosodiethylamine & 0.014 & 0.033 & 0.020 & 0.004 & 0.003 \\
\hline N-Nitrosodimethylamine & 0.010 & 0.024 & 0.015 & 0.003 & 0.002 \\
\hline N-Nitrosomorpholine & 0.014 & 0.034 & 0.021 & 0.004 & 0.003 \\
\hline N-Nitrosopyrrolidine & 0.014 & 0.034 & 0.021 & 0.004 & 0.003 \\
\hline Phenol & 15 & 95 & 58 & 71 & 47 \\
\hline Pyridine & $\overline{6}$ & 35 & 22 & 27 & 18 \\
\hline Pyrrole & 3 & 18 & 11 & 13 & 9 \\
\hline Styrene & 1 & 9 & 5 & 7 & $\overline{4}$ \\
\hline Toluene & 1 & 4 & 2 & 3 & 2 \\
\hline 3-Vinylpyridine & 7 & 46 & 28 & 35 & 23 \\
\hline m,p-Xylene & 1 & 5 & 3 & 4 & 3 \\
\hline o-Xylene & 1 & 5 & 3 & 4 & 3 \\
\hline
\end{tabular}




\subsection{RESULTS AND DISCUSSION}

\subsection{Emission Factors for Environmental Tobacco Smoke and Sidestream Smoke}

\subsubsection{Volatile N-Nitrosamines}

Four volatile N-nitrosamines (VNA) were targeted for measurement in ETS and in SS: NDMA, NDEA, NPYR and NMPH. Neither NDEA nor NMPH were found in ETS or SS samples at levels above their lower limits of detection. The findings for NDEA in SS are in agreement with what has been reported by other investigators (Brunnemann and Hoffmann, 1978). To the best of our knowledge, NMPH has not been reported in tobacco smoke by other investigators.

In addition to the NDMA and NPYR chromatographic peaks, 5 to 10 additional peaks were observed in the chromatograms of the SS samples. Most had longer retention times than the NMPH standard indicating that they were probably less volatile than NMPH. In an experiment in which the Thermosorb/N cartridge was placed in front of the filter in the SS sampling train (rather than after the filter) and, therefore, collected some particulate matter, these peaks were slightly more intense. This suggests that they may be semi-volatile and non-volatile $\mathrm{N}$-nitrosamines, including tobacco-specific N-nitrosamines. The extra peaks were not observed in the chromatograms of the ETS samples. Their absence in the ETS samples may be due to their low concentrations and/or wall losses in the chamber.

For the SS measurements, the VNA in the cartridge, filter, and SSA condensate were analyzed separately. The percentage distributions among these components are reported in Table 6.1 for each cigarette brand. The sampling and analysis of VNA was duplicated for cigarette A to assess reproducibility. It should be noted that although artifact formation on the filter is controlled by spiking the filter with $50 \mathrm{mg}$ of ascorbic acid, artifact formation within the SSA condensates cannot be controlled. Thus, some of the VNA found in the SSA may be an artifact. For SS, most of the NDMA (85\%) was found in the cartridge. Only a small fraction (5\%) was adsorbed on the filter, and the remainder (10\%) was found in the SSA condensate. For NPYR, 16\% was found in the cartridge; $47 \%$ was adsorbed on the filter; and $37 \%$ was in the SS condensate.

The emission factors for the two target VNA (NDMA and NPYR) that were present in ETS and $\mathrm{SS}$ at levels above the limits of detection are reported in Table $6.2 \mathrm{as} \mathrm{ng} / \mathrm{mg}$ of tobacco consumed and as ng/cigarette. The ng/cigarette values were calculated by normalizing the actual smoked length to the standard smoked length (Table 5.3). The mass of tobacco consumed was calculated from the mass of the tobacco per unit length (Table 5.3) and the length of the cigarette smoked. 
Table 6.1. Percentage distribution of VNA in sidestream smoke among cartridge, filter and sidestream apparatus.

\begin{tabular}{|l|c|c|c|c|c|c|}
\hline & \multicolumn{3}{|c|}{ NDMA } & \multicolumn{3}{c|}{ NPYR } \\
\hline Cigarette & Cartridge & Filter & SSA & Cartridge & Filter & SSA \\
\hline A & 81.1 & 4.1 & 14.8 & 10.7 & 48.8 & 40.5 \\
\hline $\mathrm{A}^{\text {a. }}$ & 87.5 & 4.6 & 7.9 & 11.0 & 49.2 & 39.8 \\
\hline B & 87.7 & 5.1 & 7.2 & 19.3 & 52.1 & 28.6 \\
\hline C & 77.3 & 7.0 & 15.8 & 21.2 & 42.1 & 36.6 \\
\hline D & 81.7 & 3.2 & 15.1 & 18.2 & 52.3 & 29.5 \\
\hline E & 87.6 & 3.2 & 9.2 & 12.8 & 46.2 & 41.0 \\
\hline F & 83.1 & 3.6 & 13.3 & 22.9 & 35.0 & 42.1 \\
\hline 1R4F & 91.0 & 5.7 & 3.3 & 13.1 & 50.5 & 36.4 \\
\hline & & & & & & \\
\hline Average & 84.6 & 4.6 & 10.8 & 16.2 & 47.0 & 36.8 \\
\hline Std. Dev. & 4.6 & 1.3 & 4.6 & 4.8 & 5.9 & 5.2 \\
\hline C.V. ${ }^{\text {b. }}$ & 5.4 & 28.9 & 42.3 & 29.8 & 12.5 & 14.1 \\
\hline
\end{tabular}

a. Duplicate experiment.

b. C. V.: Coefficient of Variation $=[$ Std. Dev X 100] / Average.

Table 6.2. Summary of VNA emission factors for sidestream smoke and environmental tobacco smoke from six commercial cigarettes and Kentucky reference cigarette 1R4F.

\begin{tabular}{|c|c|c|c|c|c|c|c|c|}
\hline \multirow[b]{2}{*}{ Cigarette } & \multicolumn{2}{|c|}{$\begin{array}{c}\text { NDMA } \\
\text { ng/mg tobacco }\end{array}$} & \multicolumn{2}{|c|}{$\begin{array}{c}\text { NPYR } \\
\text { ng/mg tobacco }\end{array}$} & \multicolumn{2}{|c|}{$\begin{array}{c}\text { NDMA } \\
\text { ng/cigarette }\end{array}$} & \multicolumn{2}{|c|}{$\begin{array}{c}\text { NPYR } \\
\text { ng/cigarette }\end{array}$} \\
\hline & ETS $^{2}$ & $\mathbf{S S}^{\mathrm{b}}$ & ETS $^{2}$ & SS $^{\text {b. }}$ & ETS $^{c}$ & $\mathrm{SS}^{\mathrm{d}}$ & ETS ${ }^{c}$ & $S^{d}$ \\
\hline $\mathbf{A}$ & 0.81 & 0.37 & 0.14 & 0.17 & 498 & 228 & 85 & 105 \\
\hline$A^{e}$ & 0.82 & 0.37 & 0.14 & 0.18 & 503 & 228 & 86 & 112 \\
\hline B & 1.01 & 0.58 & 0.19 & 0.24 & 572 & 330 & 105 & 138 \\
\hline C & 0.90 & 0.38 & 0.15 & 0.29 & 477 & 204 & 81 & 156 \\
\hline D & 0.70 & 0.44 & 0.16 & 0.25 & 447 & 282 & 100 & 159 \\
\hline $\bar{E}$ & 0.96 & 0.45 & 0.17 & 0.19 & 750 & 356 & 135 & 145 \\
\hline F & 0.89 & 0.38 & 0.16 & 0.20 & 645 & 277 & 118 & 148 \\
\hline 1R4F & 0.69 & $\overline{0.44}$ & 0.15 & 0.22 & 449 & 286 & 100 & 143 \\
\hline $1{ }_{1 R 4 F}{ }^{e}$ & 0.68 & - & 0.15 & - & 439 & - & 95 & - \\
\hline Average ${ }^{\mathrm{f}}$ & 0.88 & 0.43 & 0.16 & 0.22 & 565 & 280 & 104 & 142 \\
\hline Std. Dev. ${ }^{f}$ & 0.11 & 0.08 & 0.02 & 0.04 & 115 & 58 & 20 & 18 \\
\hline C. V. fo, g. & 12 & 18 & 10 & 19 & 20 & 21 & 20 & 13 \\
\hline
\end{tabular}

a. ng of VNA found in the cartridge per mg of cigarette tobacco.

b. Total ng of VNA (cartridge, filter, apparatus) emitted per mg of cigarette tobacco.

c. $n g / c i g=n g$ of VNA $x$ standard length / actual smoked length.

d. $n g / c i g=$ Total ng of VNA (cartridge, filter, apparatus) $x$ standard length / actual smoked length.

e. Duplicate experiment.

f. 1R4F omitted; $n=6$, average of duplicate measurements of cigarette $A$ used in overall average.

g. C. V.: Coefficient of Variation $=($ Std. Dev. X 100)/Average. 
Reproducibility of emission measurements. Duplicate chamber experiments were conducted for two of the cigarettes, Brand A and reference cigarette 1R4F. The duplicate measurements of the ETS VNA emission factors were in excellent agreement for both NDMA and NPYR, as shown in Table 6.2. The variabilities in the duplicate chamber measurements for these compounds were within the estimated uncertainties of the sampling and analysis method (Table 5.10), indicating that the small variations in the chamber operating conditions introduced very little additional uncertainty. The duplicate determinations of the SS emission factors made for Brand A were also in excellent agreement. Brunnemann, et al. (1980) reported the results of 4 to 5 replicate measurements of NDMA SS emission factors. The coefficients of variation for the means of their replicate measurements were $12 \%(n=4), 7.5 \%(n=5)$, and $23 \%(n=5)$ for the three cigarettes tested.

Variability among cigarette brands. The variability in the ETS emission factors (ng/cigarette) among the cigarette brands was relatively small compared to the variability in mainstream smoke emission factors for these compounds which has been reported to be as high as two orders of magnitude across brands (Brunnemann, et al., 1980). The coefficient of variation for the average of the six ETS emission factors for commercial brands was only $20 \%$ for both NDMA and NPYR. The highest ETS emission factors for NDMA and NPYR were 1.7 times higher than the lowest values. Within that range, cigarettes $\mathrm{E}$ and $\mathrm{F}$ had the highest ETS emission factors for both compounds, as might be expected since these were the longest cigarettes. The ETS emission factors for reference cigarette $1 \mathrm{R} 4 \mathrm{~F}$ were similar to those for the commercial brands.

If the ETS emission factors are compared in terms of $\mathrm{ng}$ per $\mathrm{mg}$ of tobacco consumed, the variability among brands is even smaller, with coefficients of variation of only 12 and $10 \%$ for NDMA and NPYR, respectively. The ratio of the highest to the lowest emission factor for the six brands was only about 1.4 on this basis.

The variability among the six commercial brands should reflect the specifics of the tobacco processing and nitrate content. Cigarette B was the only "light" cigarette tested and it yielded the highest emissions of VNA per mass of tobacco consumed as well as higher emission factors per cigarette than its regular counterpart, A. We note that in developing the sampling and analysis method for the VNA, we used the light counterpart of Cigarette D which was not used in the main study. The ETS emission factors for this light brand (581 ng/cig and $142 \mathrm{ng} / \mathrm{cig}$ for NDMA and NPYR, respectively) were also higher than for $\mathrm{D}$, (447 ng/cig and $100 \mathrm{ng} / \mathrm{cig}$, respectively). These differences between regular and light cigarettes are significant within the variability of sampling and analysis (Table 5.10). Such a difference is possibly due to the fact that higher proportions of stems and ribs of the tobacco leaf are used in light cigarettes. These parts 
are richer in nitrate than the rest of the leaf, and nitrate has been shown to be an important determinant of VNA yields (Brunnemann, et al., 1977; Preussmann, 1984).

The variability in the SS emission factors, expressed as ng per cigarette, for both VNA was also relatively small for the six commercial brands. There was only a factor of 1.7 difference between the highest and lowest SS emission factors for NDMA (204 to $356 \mathrm{ng} / \mathrm{cig}$ ) and a factor of 1.5 for NPYR (105 to $159 \mathrm{ng} / \mathrm{cig}$ ). The highest SS emission factors for NDMA were found for Cigarettes $E$ and B; for NPYR, the highest emission factors were for Cigarettes $D$ and $C$. The coefficients of variation of the mean emission factors ( $\mathrm{ng} / \mathrm{cig}$ or $\mathrm{ng} / \mathrm{mg}$ tobacco) for the six commercial brands were 21 and 13\% for NDMA and NPYR, respectively, which are similar to the estimated variabilities for sampling and analysis.

Comparison of SS and ETS emission factors. The NDMA emission factors ( $\mathrm{ng} / \mathrm{mg}$ tobacco) were approximately twice as high for ETS as for SS ( $p<0.001$, pairwise $t$-test). This is probably due to differences between the tobacco burning environment of the cigarette in the SSA and in the environmental chamber. Brunnemann, et al. (1977) have reported that NDMA emission factors approximately doubled when air flow through the SSA was doubled. They also reported an eight-fold increase in NDMA concentrations when 100 cigarettes were smoked in a $20-\mathrm{m}^{3}$ room with air circulation compared to the same room with limited air circulation, although the air flow conditions were not reported. In our experiments, the tobacco burning rate was slightly lower in the SSA (10 to 11 puffs per cigarette to get to the standard butt length) than in the environmental chamber ( 8 to 9 puffs per cigarette). The lower burning rate in the SSA may have been due to lower air flow around the burning cigarette cone.

In contrast to NDMA, the NPYR emission factors (ng/mg tobacco) for SS are approximately a factor of 1.4 higher than those for ETS ( $p<0.01$, pairwise $t$-test). We hypothesize that this opposite trend for NPYR, compared to NDMA, is due to some wall deposition losses of NPYR in the environmental chamber. Although, we could not measure these deposition losses, NPYR is likely to have higher wall loses because of its lower vapor pressure at room temperature $(0.72 \mathrm{~mm} \mathrm{Hg})$ compared to NDMA $(2.7 \mathrm{~mm}$ $\mathrm{Hg}$ ). For phenol with a vapor pressure of $0.3 \mathrm{~mm}$, there was evidence of deposition losses (see Section 6.1.3). The SS measurements further suggest that there may have been deposition losses of NPYR in the chamber experiments. In the SS measurements, $84 \%$ of the NPYR condensed and was deposited on the SSA interior wall and on the filter. In contrast, $85 \%$ of the NDMA was found in the vapor phase in the SS measurements.

Comparison to previously reported $N$-nitrosamine emission factors. ETS emission factors were not generally measured or reported in the older literature. Some indoor air concentrations of VNA were measured. However, since ventilation rate data were not reported, it is not possible to calculate emission 
factors from these kinds of measurements. There is one report of NDMA emission factors for a $21-\mathrm{m}^{3}$ experimental chamber (Brunnemann, et al., 1980). In these experiments, 15 to 80 cigarettes were smoked in the chamber with a 30 -port smoking machine. For the three cigarette brands tested, average NDMA emission factors were $216 \pm 26,228 \pm 8$ and $232 \pm 55 \mathrm{ng} / \mathrm{cig}$. These values are only about half of our average value of $565 \pm 115 \mathrm{ng} / \mathrm{cig}$. As discussed above, airflow conditions around the cone of the cigarette have been shown to have a substantial influence on VNA emissions and may have contributed to this difference (Brunnemann, et al., did not report air flow conditions for their chamber). Differences in the nitrate contents of the cigarettes could also explain some of the difference since more reconstituted tobacco is now used.

Brunnemann, et al. (1980) measured VNA SS emission factors for a number of Swiss and German commercial cigarettes in a SSA similar to ours but with an outer cooling jacket. Ten cigarettes were smoked using a smoking cycle and air flow rate through the SSA that were similar to our conditions. Our results for the six commercial brands are compared to the Swiss and German cigarette results in Table 6.3. For NDMA, there is very good agreement, while our NPYR average is about $70 \%$ higher. SS emission factors have also been reported for reference cigarette 1R4F. These are in good agreement with our values as shown in Table 6.3.

Table 6.3. Comparison of SS emission factors for VNA reported for American, Swiss and German cigarettes and Kentucky reference cigarette 1R4F, ng/cigarette.

\begin{tabular}{|l|c|c|c|c|}
\hline & & & \multicolumn{2}{|c|}{ Average SS Emission Factor } \\
\hline Study & Cigarettes & No. of Brands & NDMA & NPYR \\
\hline This Study & American & 6 & $280 \pm 58$ & $142 \pm 18$ \\
\hline Brunnemann, et al., 1980 & Swiss & 12 & $270 \pm 80$ & $85 \pm 32$ \\
\hline Brunnemann, et al., 1980 & German & 10 & $261 \pm 88$ & $86 \pm 28$ \\
\hline This Study & 1R4F & 1 & 286 & 143 \\
\hline Caldwell, et al., 1990 & 1R4F & 1 & 372 & 179 \\
\hline R.J. Reynolds, 1988 & 1R4F & 1 & 298 & 182 \\
\hline
\end{tabular}


For modeling indoor air exposures to VNA for Californians, we recommend using the ETS emission factors determined in this study for the six major brands of cigarettes currently on the California market. ETS rather than SS factors are recommended for use because the air flow conditions in the environmental chamber more closely simulate an indoor environment. Losses to indoor surfaces should be relatively low for NDMA, due to its high vapor pressure. For NPYR, which has a lower vapor pressure, modeled exposures are likely to be overestimated relative to actual exposures because of deposition losses to walls and other surfaces, unless these deposition losses are accurately accounted for.

\subsubsection{Aldehydes}

Table 6.4 presents the aldehyde ETS emission factors. Since we thought that there might be losses of acrolein over time due to chemical reactions, we measured aldehyde concentrations over both the first and last 100 of each experiment. The ratios of the aldehyde emission factors for the last and first samples were 1.00, 1.01 and 0.98 for formaldehyde, acetaldehyde and acrolein, respectively. Therefore, it was concluded that the aldehydes were stable over the four-hour period of the experiments and that chemical reactions of these compounds in the chamber were minimal. The values shown in the table are averages of the two samples collected in each experiment.

Reproducibility of ETS emission measurements. Duplicate chamber experiments were conducted for Brand $A$ and for reference cigarette 1R4F. The variabilities in the duplicate emission factors for formaldehyde and acetaldehyde (taken as the deviation of each value from the mean divided by the mean of the duplicates $\times 100 \%$ ) were about $12 \%$ or less and were similar to the variabilities estimated for sampling and analysis (Table 5.10). The variabilities for the duplicate emission factors for the more chemically reactive acrolein were only slightly larger, $17 \%$ for cigarette $A$ and $12 \%$ for the $1 \mathrm{R} 4 \mathrm{~F}$ cigarette.

Reproducibility of SS emission measurements. Triplicate experiments were performed for Brand A. Concentrations of formaldehyde and acrolein could not be reliably determined in these experiments due to chromatographic interferences. Therefore, replicate data are only available for acetaldehyde. For this compound, the variability in the emission factors was $4 \%$ as determined by the coefficient of variation.

Variability among cigarette brands. The variabilities in the ETS emission factors for formaldehyde and acetaldehyde were small compared to the reported variabilities in SS emission factors which range over a factor of about 700 for formaldehyde and 30 for acrolein (NRC, 1986). The coefficients of variation of the means of the ETS emission factors for formaldehyde and acetaldehyde $(\mu \mathrm{g} / \mathrm{cig}$ ) among brands of cigarettes were $27 \%$ and $22 \%$, respectively (Table 6.4 ). The ratios of the highest to the lowest emission factors $(\mu \mathrm{g} / \mathrm{cig})$ were 2 for formaldehyde and 1.4 for acetaldehyde. Ratios for the emission factors expressed in $\mathrm{ng} / \mathrm{mg}$ of tobacco were similar. 
Table 6.4. Summary of aldehyde emission factors for environmental tobacco smoke from six commercial cigarettes and Kentucky reference cigarette 1R4F.

\begin{tabular}{|c|c|c|c|c|c|c|}
\hline \multirow[b]{2}{*}{ Cigarette } & \multicolumn{3}{|c|}{ ng/mg of tobacco consumed ${ }^{2}$} & \multicolumn{3}{|c|}{$\mu \mathrm{g} /$ cigarette $^{\mathrm{b} .}$} \\
\hline & Formaldehyde & Acetaldehyde & Acrolein & Formaldehyde & Acetaldehyde & Acrolein \\
\hline $\mathbf{A}$ & 2560 & 4410 & 226 & 1570 & 2700 & 139 \\
\hline$A^{c}$ & 2540 & 4150 & 162 & 1550 & 2540 & 99.0 \\
\hline$\overline{\mathbf{B}}$ & 2170 & 3330 & 53.2 & 1230 & 1890 & 30.2 \\
\hline $\mathrm{C}$ & 1940 & 2820 & 82.2 & 1030 & 1500 & 43.7 \\
\hline $\mathbf{D}$ & 1510 & 3030 & 19.5 & 958 & 1930 & 12.4 \\
\hline $\mathbf{E}$ & 2400 & 3530 & 314 & 1880 & 2760 & 246 \\
\hline $\mathbf{F}$ & 1650 & 3040 & 90.6 & 1190 & 2200 & 65.5 \\
\hline 1R4F & 2190 & 3780 & 153 & 1420 & 2450 & 98.9 \\
\hline 1R4F ${ }^{c}$ & 1920 & 3070 & 120 & 1240 & 1990 & 77.5 \\
\hline Average $^{\text {d. }}$ & 2040 & 3340 & 126 & 1310 & 2150 & 86.0 \\
\hline Std. Dev. ${ }^{\text {d. }}$ & 412 & 525 & 109 & 349 & 477 & 87.0 \\
\hline C. $v^{\text {d., e. }}$ & 20 & 16 & 86 & 27 & 22 & 101 \\
\hline
\end{tabular}

a. ng of aldehyde emitted per mg of cigarette tobacco consumed.

b. $\mu \mathrm{g} / \mathrm{cig}=\mu \mathrm{g}$ of aldehyde $\mathrm{x}$ standard length / actual smoked length.

c. Duplicate experiments.

d. 1R4F omitted; $n=6$; average of duplicate experiments for $A$ used to calculate average among brands.

e. C. V.: Coefficient of Variation $=($ Std. Dev. X 100)/Average.

The emission factors for these two compounds in the 1R4F cigarette were very close to the averages for the six commercial brands. Variability in emission factors among brands of cigarettes was considerably larger for acrolein than for the formaldehyde and acetaldehyde. The coefficient of variation of the mean acrolein ETS emission factor ( $\mu \mathrm{g} / \mathrm{cig}$ ) was $101 \%$, and the ratio of the highest to the lowest emission factor was 20 .

Sidestream emission factors. Table 6.5 summarizes the SS emission factors for the aldehydes. Three determinations were made of the SS emission factors for cigarette A. However, difficulties were encountered in the analysis of formaldehyde and acrolein for this brand of cigarette. A relatively strong chromatographic signal appeared between the formaldehyde and acetaldehyde DNPH derivative signals. This cannot be a DNPH derivative of a carbonyl compound because there are no other $C_{1}$ or $C_{2}$ carbonyl compounds.

The interference was not present in the SS samples of the other brands. Another difference between cigarette $\mathrm{A}$ and the others was that the acrolein-DNPH peak heights for Cigarette $\mathrm{A}$ were much higher. The DNPH derivative of furfural elutes close to that of acrolein and these peaks are sometimes difficult to resolve (Schlitt and Knoppel, 1989). Since this SS emission factor could not be determined with confidence for cigarette $\mathrm{A}$, it was not reported. 
Table 6.5. Summary of aldehyde emission factors for sidestream smoke from six commercial cigarettes and Kentucky reference cigarette 1R4F.

\begin{tabular}{|c|c|c|c|c|c|c|}
\hline & \multicolumn{3}{|c|}{$\mathrm{ng} / \mathrm{mg}$ of tobacco consumed ${ }^{2}$} & \multicolumn{3}{|c|}{$\mu \mathrm{g} /$ cigarette $^{\mathrm{b}}$} \\
\hline Cigarette & Formaldehyde & Acetaldehyde & Acrolein & Formaldehyde & Acetaldehyde & Acrolein \\
\hline $\mathbf{A}$ & $\mathrm{d}$ & 2690 & d. & $\mathrm{d}$ & 1640 & d. \\
\hline$A^{c}$ & d. & 2680 & d. & d. & 1640 & d. \\
\hline$A^{c}$ & d. & 2890 & d. & d. & 1770 & $\overline{\text { d. }}$ \\
\hline B & 39.3 & 2480 & 53.3 & 22.3 & 1410 & 30.2 \\
\hline $\mathbf{C}$ & 36.2 & 2380 & 51.9 & 19.3 & 1260 & 27.6 \\
\hline$\overline{\mathbf{D}}$ & 42.5 & 2260 & 51.3 & 27.1 & 1440 & 32.7 \\
\hline $\bar{E}$ & 46.3 & 2820 & 70.6 & 36.2 & 2210 & 55.3 \\
\hline $\mathbf{F}$ & 45.3 & 2590 & 61.0 & 32.8 & 1870 & 44.1 \\
\hline 1R4F & 37.0 & 2560 & 45.3 & 24.0 & 1660 & 29.3 \\
\hline Average $^{\text {e. }}$ & 41.9 & 2590 & 58 & 27.5 & 1660 & 38 \\
\hline Std. Dev. ${ }^{\text {e }}$ & 4.2 & 202 & 8.2 & 7.0 & 279 & 12 \\
\hline C.V. ${ }^{e, f}$. & 10 & 8 & 14 & 26 & 17 & 30 \\
\hline
\end{tabular}

a. ng of aldehyde per $\mathrm{mg}$ of cigarette tobacco consumed.

b. $\mu \mathrm{g} / \mathrm{cig}=\mu \mathrm{g}$ of aldehyde $\mathrm{x}$ standard length $/$ actual smoked length.

c. Duplicate and triplicate experiments.

d. SS emission factors for formaldehyde and acrolein in SS from cigarette A could not be reliably determined due to evidence of chromatographic interferences.

e. 1R4F omitted; $n=6$ ( $n=5$ for formaldehyde and acrolein), average of triplicate samples used to calculate average across brands.

f. C. V.: Coefficient of Variation $=($ Std. Dev. X 100) /Average.

There were relatively small variations, only 8 to $14 \%$, among cigarette brands in the SS emission factors expressed in $\mathrm{ng} / \mathrm{mg}$ tobacco. Even when the lengths of the cigarettes were taken into account, the variations among brands in $\mu \mathrm{g} /$ cigarette were still relatively low, ranging from 17 to $30 \%$. These values are about twice the uncertainties due to sampling and analysis (Table 5.11). The SS emission factors for reference cigarette $1 \mathrm{R} 4 \mathrm{~F}$ fell within the ranges of the SS emission factors for the commercial cigarettes.

Comparison of ETS and SS emission factors. Table 6.6 compares the ETS and SS emission factors in $\mu \mathrm{g} / \mathrm{cig}$, for the aldehydes. The formaldehyde SS emission factors are only 2 to $3 \%$ of the ETS factors $(\mathrm{p}<0.01, \mathrm{n}=6)$. Formaldehyde is chemically reactive and undergoes polymerization at high concentrations resulting in the formation of dimmers (Bell, et al., 1987). Formaldehyde can also react with water to form methylene glycol and with ammonia to form hexamethylenetetramine (Dreyfors, et al., 1989). Conditions in the SSA, with high concentrations of water and ammonia from the combustion process, would increase the rates of these reactions of formaldehyde. Thus, lower emission factors would be measured for fresh undiluted SS samples than for ETS. Many of these reaction products of formaldehyde do not react with the DNPH reagent and, therefore, would not be evident in the chromatograms. 
Table 6.6. Comparison of ETS and SS emission factors for aldehydes, $\mu \mathrm{g} / \mathrm{cigarette.}$

\begin{tabular}{|c|c|c|c|c|c|c|}
\hline & \multicolumn{2}{|c|}{ Formaldehyde } & \multicolumn{2}{|c|}{ Acetaldehyde } & \multicolumn{2}{|c|}{ Acrolein } \\
\hline Cigarette & ETS & $\overline{\text { SS }}$ & ETS & SS & ETS & SS \\
\hline$\overline{\mathbf{A}}$ & 1560 & - & 2750 & 1683 & 119 & - \\
\hline B & 1230 & 22 & 1890 & 1410 & 30 & 30 \\
\hline C & 1030 & 19 & 1500 & 1260 & 44 & 28 \\
\hline $\mathbf{D}$ & 958 & 27 & 1930 & 1440 & 12 & 33 \\
\hline $\bar{E}$ & 1880 & 36 & 2760 & 2210 & 246 & 55 \\
\hline$\overline{\mathbf{F}}$ & 1190 & 33 & 2200 & 1870 & 66 & 44 \\
\hline$\overline{1 R 4 F}$ & 1330 & 24 & 2220 & 1660 & 88 & 29 \\
\hline Average $^{a}$ & 1310 & 27 & 2180 & 1650 & 86 & 36 \\
\hline Std. Dev. & 320 & 7 & 460 & 320 & 79 & 11 \\
\hline
\end{tabular}

a. For this table, the averages were calculated for the commercial brands plus the $1 \mathrm{R} 4 \mathrm{~F}$ cigarette.

The acetaldehyde SS emission factors are 65 to $85 \%$ of the ETS factors, and the difference is statistically significant $(\mathrm{p}<0.01, \mathrm{n}=7$ ). As in the case of formaldehyde, there may have been some reaction losses of acetaldehyde in the SSA. However, acetaldehyde is less reactive than formaldehyde. It is also possible that the lower acetaldehyde emission factor for SS than for ETS may have been due to differences in the burning chemistry of tobacco in the two environments (e.g., we observed that 10 to 11 puffs were required to smoke a cigarette to standard length in the SSA compared to only 8 or 9 in the environmental chamber).

For acrolein, the average SS emission factor was $42 \%$ of the average ETS emission factor; however, the difference is not significantly different $(p>0.2, n=6)$. The acrolein emission factors for both ETS and SS were more variable than those for formaldehyde or acetaldehyde. Acrolein, like formaldehyde, is more reactive than acetaldehyde and conditions in the SSA would be expected to lead to greater chemical losses. In addition, there may have been losses of acrolein in the sampling system. Risner and Martin (1994) recently investigated the reactions of acrolein from SS in acid solutions of DNPH and found evidence of dimerization of acrolein, as well as other reactions. Their results cannot be directly compared to ours because of differences in sampling methods but they are very suggestive. Similar reactions might have occurred on the DNPH coated Sep-Paks since acid is present. Such losses might explain the relatively high imprecision of our data for this compound.

The difficulty of obtaining accurate SS emission values for some of the more reactive aldehydes is also suggested by the NRC (1986) report in which the formaldehyde SS emission factor had a range of 
about 7 to $5,000 \mu \mathrm{g} / \mathrm{cig}$; and the acrolein emission factor had a range of 480 to $1,500 \mu \mathrm{g} / \mathrm{cig}$. For compounds which are chemically reactive there is clearly a greater potential for high variability in the SSA than in the chamber. Schlitt and Knoppel (1989) have reported experiments that demonstrate that airflow rates in the SSA have a substantial impact on the measured emission factors for aldehydes. They reported increases in total carbonyl SS emission factors from about $3,000 \mu \mathrm{g} / \mathrm{cig}$ at an air flow of $3.5 \mathrm{~L} \mathrm{~min}^{-1}$ to about $10,000 \mu \mathrm{g} / \mathrm{cig}$ at an air flow of $35 \mathrm{~L} \mathrm{~min}^{-1}$. As the air flow rate increases, both the dilution and the cigarette burning rate are affected and it would be difficult to resolve their separate influences. We previously noted that the tobacco burning rate was slightly slower in the SSA than in the chamber. The slower combustion rate suggests that somewhat less oxygen was getting to the fuel in the SSA than in the chamber.

Overall, SS emission factors for the carbonyl compounds are lower, and sometimes very much lower, than the ETS emission factors. However, with the exception of acrolein, the variability in replicate measurements and among brands of cigarettes for both SS and ETS is relatively small. Because of the large effects of dilution and air flow rates on SS emission factors (e.g., Schlitt and Knoppel, 1989) and the conditions in the SSA that lead to much greater potential for chemical reactions, we conclude that ETS emission factors for aldehydes cannot be reliably estimated from SS emission factors.

Comparison to previously reported aldehyde emission factors. Table 6.7 compares emission factors for aldehydes reported in the literature to those reported in this study. The ETS emission factors for acetaldehyde measured in this study are in very good agreement with those reported by Lofroth, et al. (1989) for a $2 \mathrm{R} 1$ reference cigarette. Our formaldehyde emission factor is only half to three-fourths of the two values reported by Lofroth, et al. (1989) but is very similar to the value reported by Jermini, et al. (1976) for 10 American cigarettes smoked in a $30-\mathrm{m}^{3}$ chamber. The variability among the reported ETS emission factors for formaldehyde is likely due to differences in the cigarettes used, numbers of cigarettes smoked and chamber ventilation rates.

In general, the reported SS emission factors for formaldehyde and acrolein (Table 6.7) are highly variable (NRC, 1986; Jermini, et al., 1976; Schlitt and Knoppel, 1989; Risner and Martin, 1994) compared to those for acetaldehyde. There is considerable evidence that the degree of dilution of SS and the air flow rate around the burning cone of the cigarette influence the measured emission factors for these reactive aldehydes. For example, the formaldehyde SS emission factor reported by Schlitt and Knoppel, (1989) is similar to the ETS factor measured in this study. Their SSA was a cylinder, 14-cm diameter, with the cigarette positioned near the bottom and air flowing from below the cigarette. Samples of SS were collected at a distance of $50 \mathrm{~cm}$ above the burning cigarette and the smoke was highly diluted and wellmixed. Thus, the reactive losses of formaldehyde would be lower and the emission factors would expected to be higher, as was observed. Also, they did not use a filter to remove particles before sampling as we did. 
Table 6.7. Comparison of ETS and SS aldehyde emission factors determined in this study to literature data.

\begin{tabular}{|c|c|c|c|c|c|}
\hline & & & \multicolumn{3}{|c|}{ Emission factors, $\mu \mathrm{g} / \mathrm{cig}(\mathbf{n})^{\mathrm{a}}$} \\
\hline Study & Experimental Conditions & ETS or SS & Formaldehyde & Acetaldehyde & Acrolein \\
\hline This study & $\begin{array}{l}20-\mathrm{m}^{3} \text { stainless steel } \\
\text { chamber; } 3 \text { cigarettes in } 30 \\
\text { min.; air exchange rate, } \\
0.029 \mathrm{~h}^{-1}\end{array}$ & ETS & $1310 \pm 349(6)$ & $2150 \pm 477(6)$ & $86 \pm 87(6)$ \\
\hline $\begin{array}{l}\text { Lofroth, et al., } \\
1989\end{array}$ & $\begin{array}{l}13.6 \mathrm{~m}^{3} \text { Plexiglas chamber; } \\
1 \mathrm{cig} / 15 \mathrm{~min} \text {. or } 1 \mathrm{cig} / 30 \\
\text { min.; air exchange rate } \\
4.05 \mathrm{~h}^{-1}\end{array}$ & ETS & $2000^{\mathrm{b}}$ & $2400^{\mathrm{b}}$ & - \\
\hline $\begin{array}{l}\text { Jermini, et al., } \\
1976\end{array}$ & $\begin{array}{l}30-\mathrm{m}^{3} \text { chamber; } \\
10 \text { American cigarettes } \\
\text { smoked }\end{array}$ & ETS & $1680^{c}$ & - & $870^{c}$ \\
\hline This study & $\begin{array}{l}\text { SS apparatus, } 225 \mathrm{~mL} \text {; air } \\
\text { flow rate } 1.5 \mathrm{~L} / \mathrm{min} \text {. }\end{array}$ & SS & $28 \pm 7(5)$ & $1660 \pm 279(6)$ & $38 \pm 12(5)$ \\
\hline $\begin{array}{l}\text { Jermini, et al., } \\
1976\end{array}$ & $\begin{array}{l}\text { 272-L chamber; } \\
1 \text { American cigarette } \\
\text { smoked }\end{array}$ & SS & - & - & $850^{c}$ \\
\hline NRC, 1986 & $\begin{array}{l}\text { SS data presented in } \\
\text { Table } 2-2 ; \mathrm{SSA} \text { air } \\
\text { flow rate } 1.5 \mathrm{~L} / \mathrm{min} \text {. }\end{array}$ & SS & $7-5,000$ & - & $480-1500$ \\
\hline $\begin{array}{l}\text { Schlitt \& } \\
\text { Knoppel, } 1989\end{array}$ & $\begin{array}{l}\text { SS apparatus, } 7.7 \mathrm{~L} \text {; air } \\
\text { flow rate, } 3.5 \mathrm{~L} / \mathrm{min} \text {. }\end{array}$ & SS & $2200,2300(2)$ & $4600,6100(2)$ & $820,880(2)$ \\
\hline $\begin{array}{l}\text { Risner \& } \\
\text { Martin, } 1994\end{array}$ & $\begin{array}{l}\text { "Fishtail chimney;" } \\
2 \text { L/min air flow through } \\
\text { apparatus; deposits on SSA } \\
\text { collected and combined } \\
\text { with impinger solutions for } \\
\text { analysis }\end{array}$ & SS & $806 \pm 59^{\mathrm{d}}$ & $1938 \pm 48^{\mathrm{d}}$ & $26 \pm 7^{\mathrm{d}}$ \\
\hline
\end{tabular}
a. $\mathrm{n}=$ number of cigarette brands.
b. 2R1 Reference cigarette.
c. Estimated from data presented in Jermini, et al., 1976.
d. Six replicate determinations of 1 R4F. 
A higher air flow rate through the apparatus is also likely to have increased the combustion rate of the cigarette, but the authors did not comment on this.

The SS emission factors reported for acrolein by Schlitt and Knoppel, (1989) and by Jermini, et al. (1976) are about an order of magnitude greater than those reported by Risner and Martin, (1994) and by us. The recent report by Risner and Martin, (1994) suggests that increased concentrations of acid in the DNPH impinger solutions used to trap aldehydes lead to increased dimerization of acrolein and that some dimerization occurred even in the absence of acid. These investigators used an aqueous solution of DNPH plus hydrochloric acid and some diglyme to keep the DNPH in solution. Schlitt and Knoppel (1989) used an aqueous solution of DNPH with phosphoric acid and acetonitrile. Jermini, et al. (1976) used direct gas injection of filtered SS into a capillary gas chromatograph for analysis. The sampler which we used was a silica Sep-Pak coated with DNPH and phosphoric acid (Kuwata, et al., 1979, 1983). This sampler has been well validated for the collection of low levels of formaldehyde and acetaldehyde but not for acrolein. Thus, we suspect that our low SS and ETS emission relative to those reported by Schlitt and Knoppel (1989) and Jermini, et al. (1976), may have been due to losses of acrolein in the sampler. There is clearly a need for the development and validation of a sampling and analysis method for acrolein at low levels using known concentrations of acrolein in air. This would require considerable effort.

In summary, the ETS emission factors for formaldehyde and acetaldehyde measured in the environmental chamber for six brands of cigarettes are recommended for use in estimating population exposures. The duplicate determinations are consistent with the estimated variability due to sampling and analysis. In addition, the sampling and analysis method for these compounds using DNPH coated Sep-Paks has been well validated (Kuwata, et al., 1979; 1983; Grosjean, 1992, and references therein). The aldehydes in diluted cigarette smoke in air appear to be chemically stable over a period of at least four hours, as indicated by the identical emission factors measured during the first and last 100 minutes of the chamber experiments. Because we suspect that there may be negative artifacts for acrolein associated with the sampling method, we do not recommend that the acrolein ETS emission factors be used for exposure estimations. Further work is needed to develop a validated sampling and analysis method for this compound.

\subsubsection{Volatile Organic Compounds}

Emission factors for twenty VOCs were measured for ETS and SS using the multisorbent sampler and GC/MS analysis. In addition to the VOCs listed in Table 3.1, emission factors were measured for pyridine, pyrrole, and 3-vinylpyridine because these compounds are potential indoor tracers of vapor-phase VOCs in ETS. The meta- and para- isomers of cresol and of xylene could not be chromatographically resolved. Therefore, these two isomers were quantified as a single combined measurement designated $\mathrm{m}, \mathrm{p}$ - 
cresol and m,p-xylene. Butyraldehyde, ethyl acrylate, ethyl acetate, 3-methyl-1-butanol and butyl acetate were not detected in any of the ETS or SS samples. In addition, 1,3-butadiene, phenol and the cresol isomers were not detected in any of the SS samples. The absence of data for some of these compounds may be due, in part, to the relatively low sample sizes for the collection of VOCs in the chamber and the SSA experiments. These sample sizes could not be increased to improve the limits of detection without overloading and contaminating the analytical system with the more abundant sample components.

For ETS, the chamber concentrations of all of the VOCs in the four sequential sampling intervals for each experiment are presented in the Appendix along with summary statistics. The concentrations of 1,3-butadiene, of 3-vinylpyridine, phenol and cresols generally had the highest coefficients of variation in each experiment. For the other VOCs, the coefficients of variation were generally $15 \%$, or less.

The chamber concentrations for 1,3-butadiene showed some evidence of increases with time in a few experiments. However, these increases were not consistent for all of the experiments. For example, airborne 1,3-butadiene levels increased over time in experiments with cigarettes A, B and D and varied irregularly in experiments with cigarettes $\mathrm{C}, \mathrm{E}, \mathrm{F}$ and $1 \mathrm{R} 4 \mathrm{~F}$. There was also greater variability in the sampling and analysis for this compound than for any of the other VOCs. We concluded that the apparent increase in concentration with time in several experiments was not significant relative to this experimental uncertainty.

The chamber concentrations of 3-vinylpyridine, phenol and cresols showed evidence of consistent decreases with time in all experiments. These concentrations declined at rates faster than the air exchange rate of the chamber, indicating additional removal probably by deposition to the chamber walls. Conversely, it was concluded that the other VOCs were stable in the chamber and did not undergo significant chemical reactions or deposition losses.

Tables 6.8 and 6.9 summarize the ETS emission factors for the 15 relatively stable VOCs and 1,3butadiene determined as $\mathrm{ng} / \mathrm{mg}$ of tobacco consumed and $\mu \mathrm{g} /$ cigarette, respectively. These emission factors were calculated using Equation (7) for each of the four samples collected beginning at elapsed times of 0, 1, 2 and 3 hours, and the averages of the four values are reported in the tables. The emission factors for 3vinylpyridine, phenol and cresols were calculated using another method described later in this section.

Reproducibility of the ETS emission measurements. Duplicate chamber experiments were conducted with cigarette $A$ to investigate the reproducibility of the ETS emissions measurements. The variabilities in the duplicate emission factors (Tables 6.8 and 6.9) for the VOCs from cigarette A (taken as the deviation of each value from the mean divided by the mean of the duplicates $\mathrm{x} 100 \%$ ) ranged generally 
Table 6.8. Summary of VOC emission factors for environmental tobacco smoke from six commercial cigarettes and Kentucky reference cigarette 1R4F, ng/mg of tobacco consumed.

\begin{tabular}{|c|c|c|c|c|c|c|c|c|c|c|c|}
\hline Cigarette & $\mathbf{A}$ & $\mathbf{A}^{*}$ & $\mathbf{B}$ & $\mathbf{C}$ & D & $\mathbf{E}$ & $\mathbf{F}$ & 1R4F & Average $^{\text {a. }}$ & Std. Dev. & C. V., ${ }^{\text {c. } \%}$ \\
\hline \multicolumn{12}{|l|}{ Compound } \\
\hline Acrylonitrile & 191 & 162 & 167 & 139 & 158 & 153 & 134 & 185 & 154 & 16.4 & 11 \\
\hline Benzene & 669 & 588 & 659 & 608 & 609 & 677 & 601 & 653 & 630 & 31.0 & 5 \\
\hline 1,3-Butadiene & 338 & 233 & 247 & 220 & 235 & 230 & 201 & 276 & 236 & 28.7 & 12 \\
\hline 2-Butanone & 432 & 432 & 455 & 451 & 451 & 495 & 425 & 585 & 451 & 24.6 & 5 \\
\hline Butyl acetate & $\mathrm{ND}^{d .}$ & ND & ND & ND & ND & ND & $\mathrm{ND}$ & ND & $<4$ & & \\
\hline Butyraldehyde & ND & ND & ND & ND & ND & ND & ND & ND & $<29$ & & \\
\hline Ethyl acetate & ND & ND & ND & $\mathrm{ND}$ & ND & ND & ND & $\mathrm{ND}$ & $<6$ & & \\
\hline Ethyl acrylate & ND & ND & ND & ND & ND & ND & ND & ND & $<5$ & & \\
\hline Ethylbenzene & 175 & 151 & 161 & 152 & 134 & 175 & 146 & 178 & 155 & 14 & $\overline{9}$ \\
\hline 3-Methyl-1-butanol & ND & ND & ND & $\mathrm{ND}$ & ND & ND & ND & ND & $\overline{<23}$ & & \\
\hline Pyridine & 828 & 614 & 706 & 653 & 514 & 850 & 533 & 989 & 663 & 126 & 19 \\
\hline Pyrrole & 725 & 653 & 642 & 669 & $\overline{508}$ & 733 & 515 & 816 & 626 & 93.6 & 15 \\
\hline Styrene & 261 & 236 & 232 & 229 & 209 & 244 & 213 & 250 & 229 & 15.9 & 7 \\
\hline Toluene & 1140 & 1000 & 1050 & 1070 & 917 & 1100 & 931 & 1130 & 1020 & 78.1 & 8 \\
\hline m,p-Xylene & 518 & 452 & 491 & 483 & 426 & 506 & 407 & 504 & 467 & 39.8 & 9 \\
\hline o-Xylene & 110 & 97.0 & 113 & 99.4 & 92.5 & 125 & 89.2 & 115 & 104 & 13.3 & 13 \\
\hline
\end{tabular}

a. To calculate the average among brands the average of the sequential measurements for each cigarette (including the duplicate measurements of brand $A$ and excluding IR4F) was used $(n=6)$.

b. Std. Dev. = standard deviation of the average.

c. C.V.: $=$ Coefficient of variation $=($ Std. Dev. $\times 100) /$ Average.

d. $\mathrm{ND}=$ Not detected; average values taken from Table 5.11

* Duplicate experiment. 
Table 6.9. Summary of VOC emission factors for environmental tobacco smoke from six commercial cigarettes and Kentucky reference cigarette 1R4F, $\mu$ g/cigarette.

\begin{tabular}{|c|c|c|c|c|c|c|c|c|c|c|c|}
\hline Cigarette & $\mathbf{A}$ & $\mathbf{A}^{*}$ & B & $\mathbf{C}$ & D & $\mathbf{E}$ & $\mathbf{F}$ & 1R4F & Average ${ }^{a}$ & Std. Dev. ${ }^{\text {b. }}$ & C. V., ${ }^{\text {c. } \%}$ \\
\hline \multicolumn{12}{|l|}{ Compound } \\
\hline Acrylonitrile & 117 & 99.1 & 94.8 & 73.8 & 85.1 & 120 & 114 & 120 & 99 & 18 & 18 \\
\hline Benzene & 409 & 360 & 374 & 323 & 383 & 530 & 441 & 423 & 406 & 71 & 18 \\
\hline 1,3-Butadiene & 207 & 143 & 140 & 117 & 128 & 180 & 170 & 179 & 152 & 27 & 18 \\
\hline 2-Butanone & 265 & 264 & 258 & 240 & 271 & 388 & 326 & 379 & 291 & 56 & 19 \\
\hline Butyl acetate & $\mathrm{ND}^{\mathrm{d}}$ & ND & ND & ND & ND & ND & ND & ND & $<3$ & & \\
\hline Butyraldehyde & ND & ND & ND & ND & ND & ND & ND & ND & $<18$ & & \\
\hline Ethyl acetate & ND & ND & ND & ND & ND & ND & ND & ND & $<4$ & & \\
\hline Ethyl acrylate & ND & ND & $\mathrm{ND}$ & ND & ND & ND & ND & ND & $<3$ & & \\
\hline Ethylbenzene & 108 & 92 & 91 & 83 & 85 & 142 & 105 & 113 & 101 & 22 & 22 \\
\hline 3-Methyl-1-butanol & ND & ND & ND & ND & ND & ND & ND & ND & $<14$ & & \\
\hline Pyridine & 507 & 376 & 401 & 347 & 340 & 666 & 372 & 641 & 428 & 122 & 29 \\
\hline Pyrrole & 444 & 400 & 364 & 356 & 328 & 574 & 368 & 529 & 402 & 90 & 22 \\
\hline Styrene & 160 & 144 & 132 & 122 & 135 & 191 & 151 & 162 & 147 & 24 & 17 \\
\hline Toluene & 698 & 614 & 596 & 567 & 593 & 860 & 663 & 732 & 656 & 107 & 16 \\
\hline m,p-Xylene & 317 & 277 & 279 & 257 & 260 & 396 & 308 & 327 & 299 & 52 & 17 \\
\hline 0 -Xylene & 67.3 & 59.4 & 63.9 & 52.9 & 56.8 & 97.8 & 66.9 & 74.7 & 66.9 & 16 & 24 \\
\hline
\end{tabular}

a. To calculate the average among brands, the average of the sequential measurements for each cigarette (including the duplicate measurements of brand $A$ and excluding 1R4F) was used $(n=6)$.

b. Std. Dev. = standard deviation of the average.

c. C.V.: $=$ Coefficient of variation $=$ (Std. Dev. $\times 100) /$ Average.

d. $\mathrm{ND}=$ Not detected; Average values taken from Table 5.11

* Duplicate experiment. 
from 5 to $8 \%$ for both the $\mu \mathrm{g} / \mathrm{cig}$ and $\mathrm{ng} / \mathrm{mg}$ values. The exceptions were pyridine and 1,3-butadiene which had variablities of 15 and $18 \%$, respectively. All of these variabilities were lower than the uncertainties estimated for sampling and analysis (Table 5.10), indicating that the small differences in chamber conditions (Table 5.4) introduced little or no additional uncertainty.

Variability of ETS emission factors among cigarette brands. The average VOC emission factors, standard deviations of the means and coefficients of variation for the six commercial brands of cigarettes are presented in Tables 6.8 and 6.9. The coefficients of variation for the emission factors expressed in ng of VOC per milligram of tobacco consumed ranged from 5 to $19 \%$ among the six brands (Table 6.8). The ratios of the highest to the lowest emission factors in $\mathrm{ng} / \mathrm{mg}$ ranged from 1.1 to 1.6 with most values falling in the range of 1.2 to 1.3 . The small variations in the $\mathrm{ng} / \mathrm{mg}$ emission factors suggest that the cigarette brands were similar with respect to the tobacco materials and processing, or that differences in these parameters are not important determinants of ETS emission factors. Brand E generally had the highest VOC emission factors while $F$ had the lowest. Both $E$ and $F$ are mentholated cigarettes. This suggests that mentholation is also not a significant factor in determining the ETS emission factors for these VOCs. The VOC emission factors for the reference cigarette were always higher than the means of the six commercial brands but fell within two standard deviations of these means.

For the VOC emission factors expressed as $\mu \mathrm{g}$ per cigarette (Table 6.9), the coefficients of variation among brands are slightly larger, ranging from $16 \%$ for toluene to $29 \%$ for pyridine, due to variations in the lengths of the cigarettes. The ratios of the highest emission factors to the lowest across cigarette brands ranged from 1.5 or 1.6 for most of the VOCs to 2.0 for pyridine. In general, cigarette E had the highest VOC emission factors while cigarette $\mathrm{C}$ had the lowest, which is consistent with their smoked lengths.

ETS Emission factors for VOCS which varied in concentration over time. Four of the VOCs (3-vinylpyridine, phenol, m,p-cresol and o-cresol) showed consistent decreases in chamber concentrations over time (see Appendix) which were greater than the variabilities due sampling and analysis. Figure 6.1 compares the temporal concentrations for two of these compounds to two other VOCs (1,3-butadiene and toluene) for the chamber experiment with cigarette B. The time-dependent concentration decreases were greater than could be accounted for by the small air exchange rate of the chamber, indicating that there were additional removal processes such as deposition onto the chamber surfaces. Removal by deposition is expected to follow a first-order exponential decay as assumed in equation (8). 
1,3-Butadiene

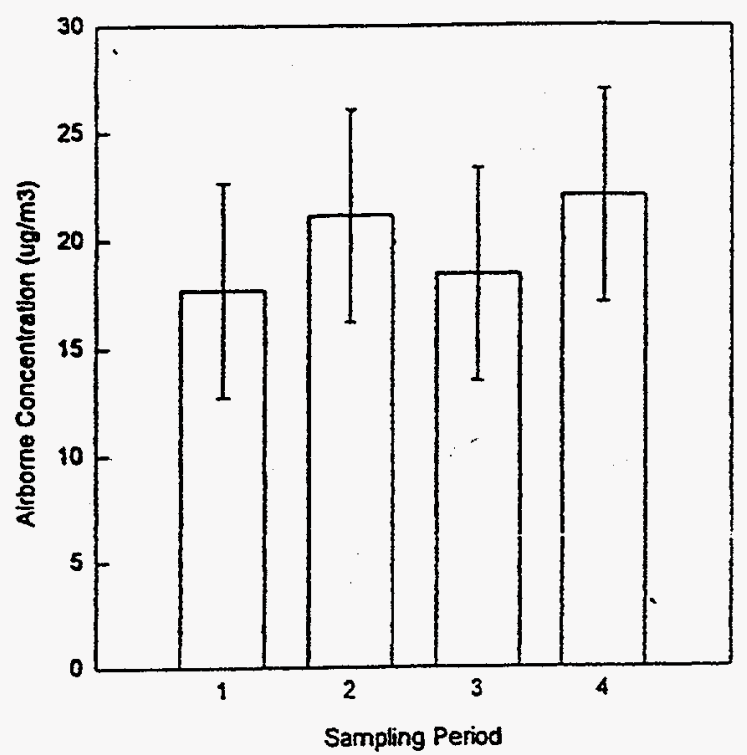

3-Vinylpyridine

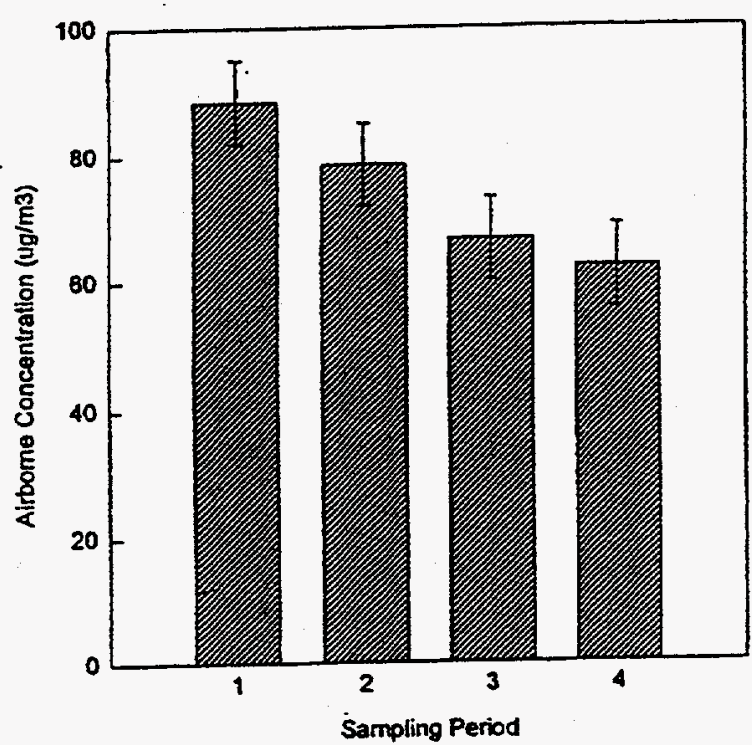

Toluene

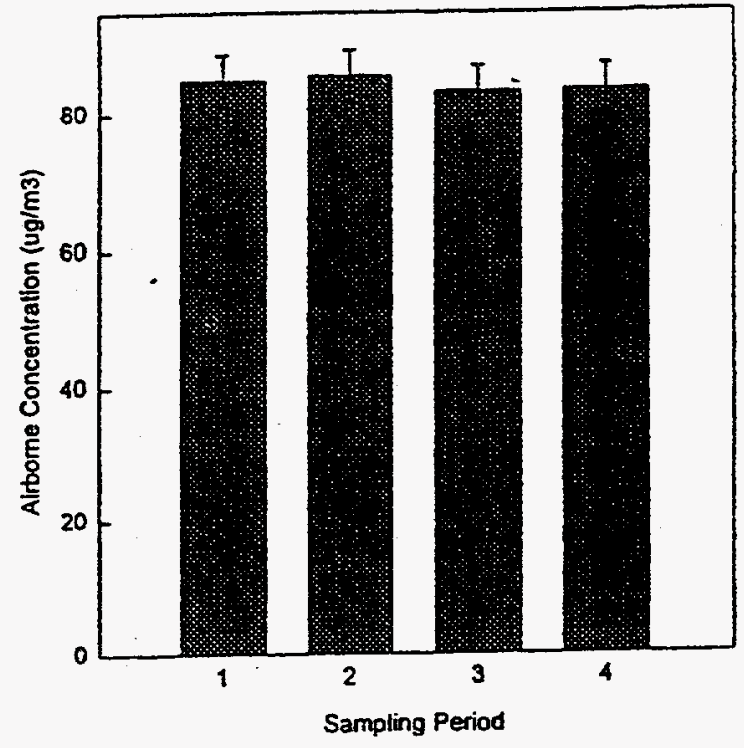

Phenol

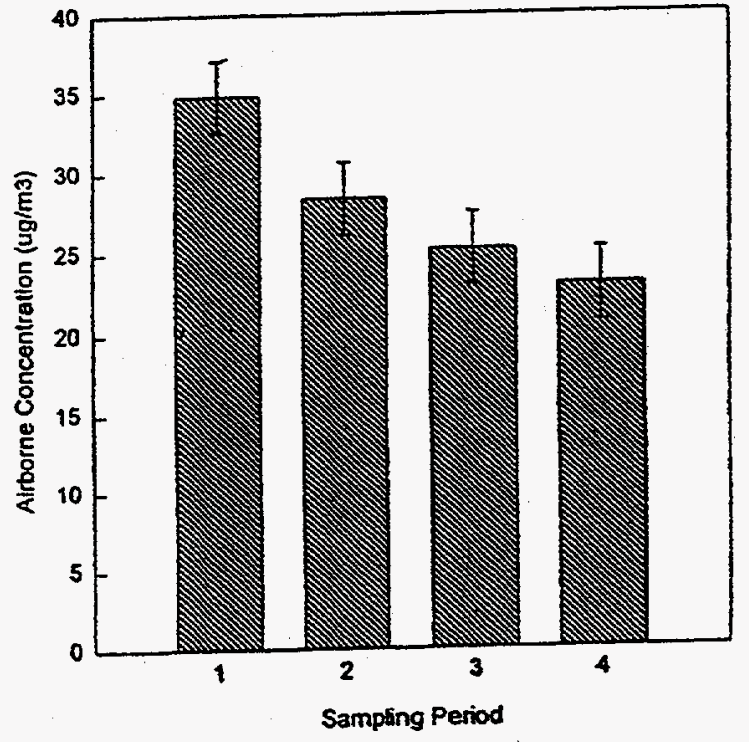

Figure 6.1. Variations in chamber concentrations of selected VOCs in ETS over time for cigarette B. 
In order to test the assumption of first-order decay, equation (8) was rearranged to equation (22),

$$
\ln C_{m}=\ln \frac{N E\left[1-e^{-(a+k)\left(t_{f}-t_{i}\right)}\right]}{\left[V(a+k)\left(t_{f}-t_{i}\right)\right]}-(a+k) t_{i} .
$$

Plots of $\ln C_{m}$ vs. $i_{i}$ for 3-vinylpyridine, phenol and the cresols declined linearly for all of the chamber experiments. As an example, plots of $\ln \mathrm{C}_{\mathrm{m}}$ vs. $\mathrm{t}_{\mathrm{i}}$ for 3-vinylpyridine are shown in Figure 6.2. Since the decay is linear, the VOC removal mechanism is a first-order process as assumed in equation ( 8 and 22). The VOC emission rates and the VOC removal rates due to wall loses for these compounds were calculated from the linear regressions. The removal rates, $k$, were determined by subtracting the air exchange rate, $\mathrm{a}$, from the slopes. The $\mathrm{k}$ values are tabulated in Table 6.10. These values are approximately inversely related to the compounds' vapor pressures. The saturation vapor pressures of phenol, o-cresol and $\mathrm{m}, \mathrm{p}$-cresol at $25^{\circ} \mathrm{C}$ are $0.055,0.030$ and $0.012 \mathrm{~mm} \mathrm{Hg}$, respectively, while the deposition rate constants for these compounds are $0.17,0.27$ and $0.24 \mathrm{~h}^{-1}$, respectively. The deposition rate constant for the more volatile 3-vinylpyridine was $0.09 \mathrm{~h}^{-1}$ (no vapor pressure data was found for this compound). The behavior of these compounds is consistent with removal by deposition onto the chamber surfaces. It should be noted that the deposition rate constants apply only to our environmental chamber and additional work is needed to

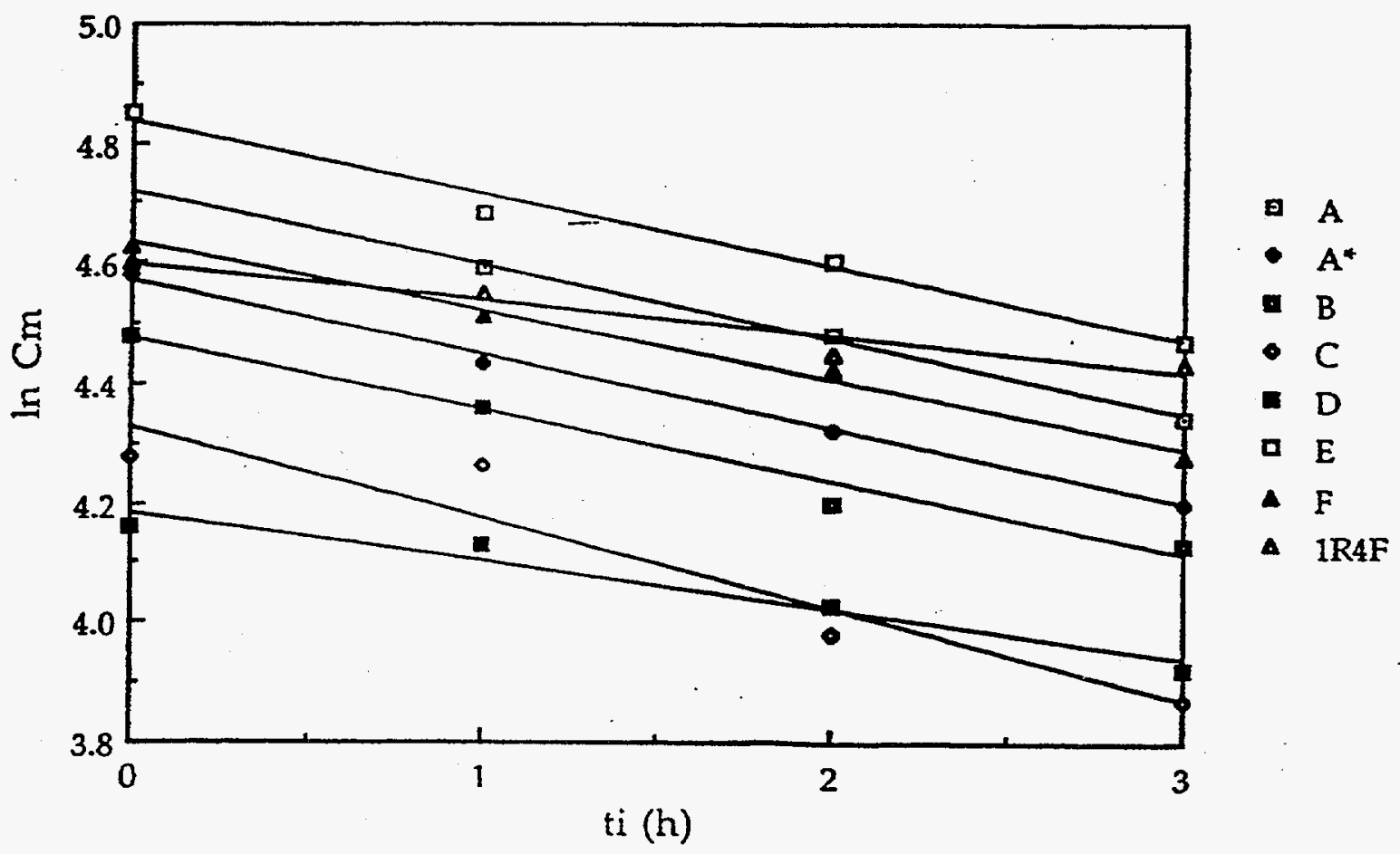

Figure 6.2. Plots of In measured concentration versus initial sampling time for 3-vinylpyridine in chamber experiments with six commercial cigarettes and reference cigarette $1 \mathrm{R} 4 \mathrm{~F}$. 
Table 6.10. Calculated $k$ values for 3-vinylpyridine, phenol and cresols from six commercial cigarettes and Kentucky reference cigarette 1R4F.

\begin{tabular}{|c|c|c|c|c|}
\hline & \multicolumn{4}{|c|}{$k, h^{-1}$} \\
\hline Cigarette & 3-VNP ${ }^{\mathrm{a}}$ & Phenol & o-Cresol & m,p-Cresol \\
\hline $\mathbf{A}$ & 0.096 & 0.21 & 0.20 & 0.14 \\
\hline$A^{\text {b. }}$ & 0.096 & 0.14 & 0.29 & 0.23 \\
\hline B & 0.092 & 0.10 & 0.27 & 0.28 \\
\hline $\bar{C}$ & 0.122 & 0.26 & 0.48 & 0.22 \\
\hline D & 0.053 & 0.13 & 0.18 & 0.17 \\
\hline $\mathbf{E}$ & 0.093 & 0.18 & 0.24 & 0.34 \\
\hline $\mathbf{F}$ & 0.085 & 0.15 & 0.23 & 0.27 \\
\hline 1R4F & 0.032 & 0.14 & 0.38 & 0.24 \\
\hline Average $^{c}$ & 0.090 & 0.17 & 0.27 & 0.24 \\
\hline Std. Dev. ${ }^{c}$ & 0.022 & 0.05 & 0.10 & 0.07 \\
\hline C.V. ${ }^{c, d .}$ & 25 & 31 & 38 & 27 \\
\hline
\end{tabular}

a. 3-VNP: 3-vinylpyridine.

b. Duplicate experiment.

c. IR4F omitted; $\mathrm{n}=6$.

d. C. V.: Coefficient of Variation $=($ Std. Dev. X 100) $/$ Average.

obtain information on deposition to surfaces in real buildings for use in models. However, our results provide evidence that these compounds are highly likely to be lost to indoor surfaces.

The deposition rate constants were combined with the air exchange rate to calculate the corrected emission factors for 3-vinylpyridine, phenol and the cresols. For each compound in each experiment, the time zero intercept (cessation of smoking) of a plot of $\ln \mathrm{C}_{\mathrm{m}}$ vs. $\mathrm{t}_{\mathrm{i}}$ was taken as the corrected factor. These corrected emission factors are presented in Table 6.11 in terms of $\mathrm{ng} / \mathrm{mg}$ of tobacco consumed and in Table 6.12 in terms of $\mu \mathrm{g} / \mathrm{cig}$. The variability in the duplicate corrected emission factors in $\mu \mathrm{g} / \mathrm{cig}$ for the four compounds from Cigarette A ranged from 2 to $30 \%$. The coefficients of variation of the emission factors for these four VOCs among cigarette brands in $\mathrm{ng} / \mathrm{mg}$ ranged from 16 to $20 \%$ and were only slightly greater than those for the VOCs which did not show evidence of wall losses (Table 6.8). The coefficients of variation in the $\mu \mathrm{g} / \mathrm{cig}$ for the corrected emission factors ranged from 14 to $31 \%$.

Also shown in Tables 6.11 and 6.12 are the corresponding emission factors for these compounds calculated without making the corrections for deposition losses. The uncorrected values are factors of 0.83 to 0.60 lower than the corrected values due the decays in the concentrations with time. 
Table 6.11. Corrected and uncorrected ETS emission factors for 3-vinylpyridine, phenol and cresols from six commercial cigarettes and Kentucky reference cigarette 1R4F, ng/mg tobacco consumed.

\begin{tabular}{|c|c|c|c|c|c|c|c|c|}
\hline & \multicolumn{4}{|c|}{ Corrected Emission Factor, ng/mg } & \multicolumn{4}{|c|}{ Uncorrected Emission Factor, ng/mg } \\
\hline Cigarette & 3-VNP ${ }^{2}$ & Phenol & o-Cresol & m,p-Cresol & 3-VNP ${ }^{2}$ & Phenol & o-Cresol & m,p-Cresol \\
\hline $\mathbf{A}$ & 1,300 & 653 & 67.9 & 141 & 1,030 & 422 & 41.7 & 101 \\
\hline$A^{b .}$ & 1,120 & 483 & 70.8 & 139 & 931 & 392 & 42.2 & 91.4 \\
\hline $\mathbf{B}$ & 1,080 & 421 & 63.5 & 145 & 917 & 345 & 39.5 & 88.5 \\
\hline $\bar{C}$ & 1,010 & 403 & 59.4 & 96.5 & 803 & 252 & 27.2 & 65.1 \\
\hline$\overline{\mathbf{D}}$ & 715 & 368 & 50.2 & 96.9 & 846 & 304 & 26.5 & 77.3 \\
\hline $\bar{E}$ & 1,140 & 458 & 48.3 & 160 & 948 & 328 & 31.0 & 87.5 \\
\hline $\mathbf{F}$ & 1,000 & 398 & 40.0 & 127 & 644 & 298 & 35.8 & 70.8 \\
\hline 1R4F & 1,050 & 368 & 59.1 & 106 & 988 & 284 & 32.0 & 69.6 \\
\hline Average $^{c}$ & 1,030 & 436 & 55.1 & 127 & 856 & 322 & 33.7 & 80.9 \\
\hline Std. Dev. ${ }^{c}$ & 171 & 71.0 & 10.9 & 25.9 & 123 & 52.1 & 6.44 & 11.8 \\
\hline C.V. ${ }^{c, d}$ & 17 & 16 & 20 & 20 & 14 & 16 & 19 & 15 \\
\hline
\end{tabular}
a. 3-VNP: 3-vinylpyridine.
b. Duplicate experiment.
c. 1R4F omitted; $n=6$.
d. C. V.: Coefficient of Variation $=($ Std. Dev. X 100) $/$ Average

Table 6.12. Corrected and uncorrected ETS emission factors for 3-vinylpyridine, phenol and cresols from six commercial cigarettes and Kentucky reference cigarette 1R4F, $\mu \mathrm{g} /$ cigarette.

\begin{tabular}{|l|c|c|c|c|c|c|c|c|}
\hline & \multicolumn{4}{|c|}{ Corrected Emission Factor, $\boldsymbol{\mu g}$ /cig } & \multicolumn{3}{c|}{ Uncorrected Emission Factor, $\mu$ g/cig } \\
\hline Cigarette & $\mathbf{3 - V N \mathbf { P } ^ { 2 }}$ & Phenol & o-Cresol & m,p-Cresol & 3-VNP & Phenol & o-Cresol & m,p-Cresol \\
\hline A & 796 & 400 & 41.5 & 86.1 & 628 & 258 & 25.5 & 61.6 \\
\hline A $^{\text {b. }}$ & 685 & 295 & 43.4 & 84.8 & 570 & 240 & 25.8 & 55.9 \\
\hline B & 621 & 242 & 36.5 & 83.1 & 521 & 196 & 22.5 & 50.3 \\
\hline C & 542 & 217 & 32.0 & 51.9 & 427 & 134 & 14.5 & 34.7 \\
\hline D & 455 & 235 & 32.0 & 61.7 & 410 & 190 & 22.8 & 45.1 \\
\hline E & 889 & 358 & 37.8 & 125 & 742 & 257 & 24.3 & 68.5 \\
\hline F & 724 & 288 & 29.0 & 91.6 & 612 & 220 & 19.1 & 55.9 \\
\hline 1R4F & 683 & 238 & 38.3 & 68.7 & 640 & 184 & 20.8 & 45.1 \\
\hline & & & & & & & & \\
\hline Average & 662 & 281 & 34.9 & 83.1 & 552 & 207 & 21.5 & 52.2 \\
\hline Std. Dev. & 155 & 60.6 & 4.91 & 25.5 & 125 & 45.0 & 4.05 & 11.7 \\
\hline C.V. c.d. & 23 & 22 & 14 & 31 & 23 & 24 & 19 & 22 \\
\hline
\end{tabular}
a. 3-VNP: 3-vinylpyridine.
b. Duplicate experiment.
c. 1R4F omitted; $\mathrm{n}=6$.
d. C. V.: Coefficient of Variation $=($ Std. Dev. X 100)/Average. 
Tracers of VOCs from ETS. Nicotine has been widely used as a tracer of exposures to ETS particles. However, this compound deposits on indoor surfaces and, therefore, is not an ideal tracer of the many vapor-phase VOCs from ETS. As part of this project we measured emission factors for three nitrogen-containing VOCs, pyridine, pyrrole, and 3-vinylpyridine, as potential tracers for vapor-phase compounds from ETS. The National Research Council (NRC, 1986) lists four criteria for an ETS tracer: 1) uniqueness to tobacco smoke; 2) easily detected at low smoking rates; 3) similar emission rates across different tobacco products; and 4) consistent proportions to other ETS compounds for different environments and tobacco products. The three compounds were selected because they contain nitrogen and, based on emissions data reported in the literature and measurements of VOCs in indoor environments, other indoor sources do not emit such compounds. Thus, they appear to be unique to tobacco. All three compounds can be easily detected at low smoking rates, although they are not as abundant in ETS as nicotine. The coefficients of variation for the pyridine, pyrrole, and 3-vinylpyridine emission factors $(\mu \mathrm{g} / \mathrm{cig})$ were 29,22 and $23 \%$ respectively, for the six commercial brands of cigarettes tested. The ratios of the highest to lowest emission factors were about 2 or less. Thus, all three compounds meet the third criterion reasonably well. In order to trace the vapor-phase components of ETS, the selected compound must also exhibit indoor behavior similar to that of the vapor-phase compounds it traces. Based on our measurements, 3-vinylpyridine did not meet this criterion since it apparently deposited onto surfaces in the chamber over the period of the experiments. Thus, chemical element mass-balance estimates of the contribution of ETS to indoor concentrations of vapor-phase VOCs with multiple sources (e.g., all of the compounds in Table 3.1) should be based on their ratios to the pyridine and/or pyrrole as the tracer compounds. The emission factors that we measured in this study for these two compounds and for the other VOCs provide the ETS source data needed in the mass-balance modeling approach.

Comparison of ETS and SS emission factors. Tables 6.13 and 6.14 summarize the VOC emission factors for sidestream smoke. The VOCs that were below their lower limits of detection for the ETS samples, butyl acetate, butyraldehyde, ethyl acetate, ethyl acrylate and 3-methyl-1-butanol, were also below their detection limits in the SS samples. In addition, 1,3-butadiene, $\mathrm{m}, \mathrm{p}$-cresol, o-cresol and phenol were below their detection limits in the SS samples. The 1,3-butadiene is suspected to have undergone reaction losses in the SSA. The cresols and phenol, which are relatively stable chemically, are suspected to have been lost via condensation to the SSA walls and/or filter. These compounds have the lowest vapor pressures of the target VOCs.

Triplicate determinations of the SS emission factors for cigarette A were made to evaluate their reproducibility. The coefficients of variation for the triplicate determinations in $\mu \mathrm{g} / \mathrm{cig}$ ranged from $4 \%$ for pyrrole to $18 \%$ for 3 -vinylpyridine; most were about 5 to $6 \%$. Thus, these uncertainties fell within the estimated variabilities due to sampling and analysis (Table 5.11). 
The SS emission factors for the VOCs, reported as ng per mg of tobacco consumed (Table 6.13) did not vary significantly among the cigarette brands, i.e., the coefficients of variation among brands were $18 \%$ or less. The emission factors reported as $\mu \mathrm{g}$ per cigarette (Tables 6.14 ) had somewhat higher coefficients of variation, ranging between $17-28 \%$, since the cigarettes varied in length. The variations in the $\mathrm{ng} / \mathrm{mg}$ emission factors were only slightly greater than the estimated experimental uncertainties, suggesting the brands were similar with respect to tobacco materials and processing, or that differences in these parameters are not important determinants of SS emission factors.

The average ETS and SS emission factors for the VOCs that were above the lower limits of detection are compared in Table 6.15. The ETS and SS emission factors were compared using a paired ttest. For 9 of the 11 VOCs, the ETS emission factors were significantly greater than the SS emission factors ( $p<0.01$ ), while for pyridine and pyrrole, the ETS and SS emission factors were not significantly different within the variability of the measurements. The ETS/SS ratio of the average emission factors for pyrrole is very close to one, and the ratio for pyridine is 1.16 . The other VOCs have ratios ranging from 1.2 to 2.2 (3-vinylpyridine).

The basic design for the SSA was first described 30 years ago (Neurath and Ehmke, 1964). The less sensitive analytical methods then in use required collection of quite large samples and smoking of muitiple cigarettes. When many cigarettes are smoked in the SSA to provide a single sample, condensation losses to walls probably constitute a smaller percentage of the sample than in the case in which a single cigarette is smoked and only a small fraction of the emissions is collected. As noted earlier, the SS was highly diluted with nitrogen gas and then only a very small fraction of the total flow was collected with the multisorbent sampler, i.e., $0.03 \%$, so that the analytical system would not be overloaded with sample. For VOCs that were likely to react (1,3-butadiene) or condense in the SSA (phenol, cresols), very little of the emissions would be expected to reach the multisorbent sampler. For most of the other VOCs (Table 6.15) the reasons for the consistently lower values for the SS emission factors are not known; however, many are presumably related to differences in the experimental configuration.

The SSA is not optimal for estimating emission values since it is not possible to simulate realworld dilution in a $225-\mathrm{mL}$ apparatus without substantially affecting the airflow around the burning cone of the cigarette. Other designs for a SSA may be more appropriate. For example, the SSA used by Schlitt and Knoppel (1989) for aldehyde measurements may be able to provide emission factors that are closer to those measured in an environmental chamber but with less labor and cost. This bench-scale apparatus has a volume of $7.7 \mathrm{~L}$ and the sample is collected $50-\mathrm{cm}$ above the cigarette. This allows substantial dilution of the SS and reduces the probability of chemical reactions and condensation. Future efforts to design 
Table 6.13. Summary of VOC emission factors for sidestream smoke from six commercial cigarettes and Kentucky reference cigarette 1R4F, ng/mg of tobacco consumed.

\begin{tabular}{|c|c|c|c|c|c|c|c|c|c|c|c|c|}
\hline Cigarette & $\mathbf{A}$ & $\mathbf{A}^{*}$ & $A^{* *}$ & B & C & $\bar{D}$ & $\mathbf{E}$ & $\mathbf{F}$ & 1R4F & Average $^{\mathbf{a}^{\circ}}$ & Std. Dev. & C. V., ${ }^{c .} \%$ \\
\hline \multicolumn{13}{|l|}{ Compound } \\
\hline Acrylonitrile & 99.4 & 83.2 & 118.7 & 97.9 & 98.4 & 103 & 132 & 102 & 104 & 106 & 13.1 & 12 \\
\hline Benzene & 306 & 309 & 367 & 340 & 352 & 380 & 460 & 417 & 315 & 379 & 50.9 & 13 \\
\hline 1,3-Butadiene & $\mathrm{ND}^{\mathrm{d}}$ & ND & ND & ND & ND & ND & ND & ND & ND & $<87$ & & \\
\hline 2-Butanone & 226 & 242 & 267 & 254 & 301 & 347 & 344 & 325 & 312 & 303 & 44.4 & 15 \\
\hline Butyl acetate & ND & ND & ND & ND & ND & ND & ND & ND & ND & $<3$ & & \\
\hline Butyraldehyde & ND & ND & ND & ND & ND & ND & ND & ND & ND & $<22$ & & \\
\hline m,p-Cresol & ND & ND & ND & ND & ND & ND & ND & ND & ND & $<27$ & & \\
\hline o-Cresol & ND & ND & ND & ND & ND & ND & $\mathrm{ND}$ & ND & ND & $<27$ & & \\
\hline Ethyl acetate & ND & ND & ND & ND & ND & ND & ND & ND & ND. & $<5$ & & \\
\hline Ethyl acrylate & ND & ND & ND & ND & ND & ND & ND & ND & ND & $<4$ & & \\
\hline Ethylbenzene & 110 & 102 & 129 & 128 & 128 & 131 & 143 & 134 & 138 & 130 & 9.6 & 7 \\
\hline 3-Methyl-1-butanol & ND & ND & ND & ND & ND & ND & ND & ND & ND & $<17$ & & \\
\hline Phenol & ND & ND & ND & ND & ND & ND & ND & ND & ND & $<71$ & & \\
\hline Pyridine & 517 & 543 & 686 & 582 & 593 & 642 & 677 & 587 & 606 & 611 & 39.7 & 7 \\
\hline Pyrrole & 522 & 486 & 527 & 684 & 610 & 662 & 708 & 628 & 612 & 634 & 69.7 & 11 \\
\hline Styrene & 130 & 130 & 159 & 148 & 151 & 168 & 176 & 158 & 155 & 157 & 13.4 & 9 \\
\hline Toluene & 688 & 694 & 802 & 769 & 789 & 848 & 808 & 789 & 794 & 788 & 40.0 & 5 \\
\hline 3-Vinylpyridine & 318 & 359 & 449 & 431 & 536 & 446 & 554 & 444 & 459 & 464 & 67.8 & 15 \\
\hline $\mathrm{m}, \mathrm{p}$-Xylene & 291 & 302 & 347 & 327 & 336 & 347 & 418 & 333 & 345 & 346 & 37.1 & 11 \\
\hline o-Xylene & 57.2 & 55.7 & 70.9 & 63.3 & 66.0 & 67.2 & 78.2 & 70.0 & 70.5 & 67.7 & 5.99 & 9 \\
\hline
\end{tabular}

a. To calculate the average among brands (excluding 1R4F), the average of the triplicate measurements of brand $A$ was used.

b. Std. Dev. = standard deviation of the average.

c. C.V.: Coefficient of variation $=($ Std. Dev. $\times 100) /$ Average

d. $\mathrm{ND}=$ Not detected; average values taken from Table 5.11

* Duplicate experiment; $* *$ triplicate experiment. 
Table 6.14. Summary of VOC emission factors for sidestream smoke from six commercial cigarettes and Kentucky reference cigarette 1R4F, $\mu \mathrm{g} /$ cigarette.

\begin{tabular}{|c|c|c|c|c|c|c|c|c|c|c|c|c|}
\hline Cigarette & $\mathbf{A}$ & $\dot{A} *$ & $A^{* *}$ & B & $\mathbf{C}$ & D & $\mathbf{E}$ & $\mathbf{F}$ & 1R4F & Average $^{\mathrm{a} .}$ & Std. Dev. & C. V., ${ }^{\mathrm{c} .} \%$ \\
\hline \multicolumn{13}{|l|}{ Compound } \\
\hline Acrylonitrile & 60.8 & 65.5 & 72.6 & 54.7 & 52.4 & 65.4 & 103 & 73.7 & 67.4 & 69.3 & 18.5 & 27 \\
\hline Benzene & 187 & 202 & 225 & 190 & 187. & 242 & 360 & 302 & 204 & 248 & 69.9 & 28 \\
\hline 1,3-Butadiene & $\mathrm{ND}^{\mathrm{d}}$ & ND & ND & ND & $\mathrm{ND}$ & ND & ND & ND & ND & $<58$ & & \\
\hline 2-Butanone & 138 & 148 & 163 & 144 & 160 & 221 & 270 & 235 & 202 & 197 & 52.2 & 27 \\
\hline Butyl acetate & ND & ND & ND & ND & ND & ND & ND & ND & ND & $<2$ & & \\
\hline Butyraldchyde & ND & ND & ND & ND & ND & ND & ND & ND & ND & $<14$ & & \\
\hline $\mathrm{m}, \mathrm{p}$-Cresol & ND & ND & ND & ND & ND & ND & ND & ND & ND & $<18$ & & \\
\hline o-Cresol & $\mathrm{ND}$ & ND & ND & ND & ND & ND & ND & ND & ND & $<18$ & & \\
\hline Ethyl acetate & $\mathrm{ND}$ & $\mathrm{ND}$ & $\mathrm{ND}$ & ND & ND & ND & ND & ND & ND & $<3$ & & \\
\hline Ethyl acrylate & ND & ND & $\mathrm{ND}$ & ND & ND & ND & ND & ND & ND & $<3$ & & \\
\hline Ethylbenzene & 67 & 72 & 79 & 72 & 68 & 84 & 112 & 97 & 89 & 84 & 17 & 20 \\
\hline 3-Methyl-1-butanol & ND & ND & ND & ND & ND & ND & ND & ND & ND & $<11$ & & \\
\hline Phenol & ND & ND & ND & ND & ND & ND & ND & ND & ND & $<47$ & & \\
\hline Pyridine & 316 & 340 & 420 & 325 & 315 & 409 & 530 & 425 & 393 & 394 & 79.7 & 22 \\
\hline Pyrrole & 319 & 344 & 322 & 382 & 325 & 422 & 554 & 454 & 397 & 411 & 86.7 & 21 \\
\hline Styrene & 79.3 & 85.5 & 97.5 & 82.9 & 80.2 & 107 & 138 & 115 & 101 & 101.6 & 22.4 & 22 \\
\hline Toluene & 421 & 453 & 491 & 429 & 420 & 540 & 632 & 571 & 514 & 508 & 86.3 & 17 \\
\hline 3-Vinylpyridine & 194 & 209 & 274 & 241 & 285 & 284 & 433 & 322 & 297 & 298 & 74.4 & 25 \\
\hline m,p-Xylene & 178 & 191 & 212 & 183 & 179 & 221 & 327 & 241 & 223 & 224 & 55.9 & 25 \\
\hline o-Xylene & 35.0 & 37.7 & 43.4 & 35.4 & 35.1 & 42.8 & 61.2 & 50.6 & 45.7 & 44.0 & 10.2 & 23 \\
\hline
\end{tabular}

a. To calculate the average among brands (excluding IR4F), the average of the triplicate measurements of brand $A$ was used.

b. Std. Dev. $=$ standard deviation of the average.

c. C.V.: Coefficient of variation $=($ Std. Dev. $x$ 100) $/$ Average

d. $\mathrm{ND}=$ Not detected; average values taken from Table 5.11.

* Duplicate experiment; ** triplicate experiment. 
Table 6.15 Comparison of average ETS and SS emission Factors for eleven VOCs.

\begin{tabular}{|c|c|c|c|}
\hline Compound & $\begin{array}{c}\text { ETS Emission Factors, } \\
\mu \mathrm{g} / \mathrm{cig} \\
\text { Average } \pm \text { Std. Dev. }{ }^{2}\end{array}$ & $\begin{array}{l}\text { SS Emission Factors, } \\
\quad \mu \mathrm{g} / \mathrm{cig} \\
\text { Average } \pm \text { Std. Dev. }\end{array}$ & $\begin{array}{l}\text { ETS Emission Factor is } \\
\text { Significantly Different } \\
\text { from SS Emission } \\
\text { Factor }(p<0.01)^{b .} \\
\end{array}$ \\
\hline Acrylonitrile & $102 \pm 18$ & $69 \pm 17$ & Yes; ETS $>$ SS \\
\hline Benzene & $408 \pm 66$ & $241 \pm 66$ & Yes; ETS $>$ SS \\
\hline 2-Butanone & $304 \pm 61$ & $197 \pm 48$ & Yes; ETS>SS \\
\hline Ethylbenzene & $103 \pm 20$ & $85 \pm 16$ & Yes; ETS >SS \\
\hline Pyridine & $458 \pm 138$ & $394 \pm 73$ & No. \\
\hline Pyrrole & $420 \pm 95$ & $409 \pm 79$ & No. \\
\hline Styrene & $149 \pm 23$ & $102 \pm 21$ & Yes; ETS $>S S$ \\
\hline Toluene & $667 \pm 102$ & $509 \pm 79$ & Yes; ETS>SS \\
\hline 3-Vinylpyridine & $665 \pm 142$ & $298 \pm 68$ & Yes; ETS $>$ SS \\
\hline $\mathrm{m}, \mathrm{p}$-Xylene & $303 \pm 48$ & $224 \pm 51$ & Yes; ETS>SS \\
\hline o-Xylene & $68 \pm 15$ & $44 \pm 9$ & Yes; ETS>SS \\
\hline
\end{tabular}

a. Average of six commercial brands plus $\operatorname{RR} 4 \mathrm{~F}(\mathrm{n}=7)$.

b. Paired t-test.

a bench-scale SSA for simulating ETS should also consider the air velocity around the burning cone of the cigarette and try to simulate indoor environments in this regard.

Comparison to previously reported emission factors. In making comparisons to literature data, it should be kept in mind that brand selection and representativeness varies widely among the studies. In most studies, only a few brands are represented, often selected on the basis of mainstream emissions characteristics, e.g., high and low tar. In our study, brands were selected based on market shares since a major objective was to develop ETS emission factors that could be used to estimate population exposures.

Of the 20 VOCs that were measured in this study, five VOCs, which had not been previously measured, were below the limits of detection. These were butyraldehyde, butyl acetate, ethyl acetate, ethyl acrylate, and 3-methyl-1-butanol. For two of the measured VOCs, acrylonitrile and ethylbenzene, we did not find any reported emission factors in the literature. Average emission factors (six commercial brands) for the 13 remaining VOCs are compared to available literature data in Table 6.16.

ETS emission factors for seven compounds can be compared directly to ETS literature values. For 1,3-butadiene, the ETS emission factor we measured lies between the substantially different values reported by Brunnemann, et al. (1990) and Lofroth et al. (1989). For 2-butanone, our ETS emission factor is only 
$38 \%$ of the value reported by Jermini, et al. (1976). For the other five compounds, benzene, styrene, toluene, m-,p-xylene and o-xylene, our ETS emission values are within a factor of two or less of those reported in the literature, despite substantial differences in the experimental conditions, e.g., chamber size, flow rates, cigarette brands, smoking patterns, numbers of cigarettes smoked, and sampling and analysis methods.

SS emission factors for six compounds can be compared directly to SS literature values. In this study, 1,3-butadiene was below the limit of detection which is in disagreement with the results reported by Brunnemann et al. (1990). Our pyridine SS emission factor lies within the large range of values summarized by Eatough et al. (1990). Reasonably good agreement was obtained between our values and the literature values for benzene, pyrrole, toluene and 3-vinylpyridine.

One VOC for which there appears to be a substantial difference among reported values is $1,3-$ butadiene. This is the most reactive of the VOCs measured, and it also has the highest experimental uncertainties associated with its sampling and analysis. The ETS emission factor reported by Lofroth, et al. (1989) is 2.6 times greater than the average we measured in our chamber. Differences in smoking protocols may explain some of this difference. In the experiments reported by Lofroth, et al., cigarettes were smoked continuously during sampling; thus, they were sampling freshly generated ETS. In our experiments, 3 cigarettes were first smoked, then sampling was initiated. Therefore, if there were chemical reaction losses during the 30-minute smoking period of our experiments, we would not have observed them.

Brunnemann, et al. (1990) measured 1,3-butadiene in SS and in a $16-\mathrm{m}^{3}$ chamber under ventilated and unventilated conditions. The SS emission factors which they measured for 6 different cigarettes (including 1R4F) ranged from 207 to $361 \mu \mathrm{g} / \mathrm{cig}$ (average $=248 \mu \mathrm{g} / \mathrm{cig}$ ). In the $16-\mathrm{m}^{3}$ unventilated chamber (described as a test laboratory) in which 5 cigarettes were simultaneously smoked, they measured an air concentration of $9.5 \mu \mathrm{g} / \mathrm{m}^{3}$ for 1,3-butadiene. They noted that the ratio of 1,3-butadiene to benzene (which is much more chemically stable) was 1.9 in SS. In the unventilated chamber, the ratio was 1:10; and in the chamber ventilated 12 air changes per hour, the ratio was only 1:22. They did not calculate emission factors from the chamber studies. However, if we assume that the air exchange rate in the unventilated chamber was close to zero, then the emission factor for 1,3-butadiene under these conditions was about 30 $\mu \mathrm{g} / \mathrm{cig}$, compared to an average of $249 \mu \mathrm{g} / \mathrm{cig}$ for SS. The emission factor for 1,3-butadiene in the ventilated chamber was also estimated in to be about $30 \mu \mathrm{g} / \mathrm{cig}$. These results suggested to them that substantial chemical reaction losses occurred in the chamber. They did not, however, measure changes in air concentrations over time. 
Table 6.16. Comparison of the VOC emission factors determined in this study ${ }^{\text {a. }}$ to literature data.

\begin{tabular}{|c|c|c|c|c|}
\hline Compound & Study & ETS or SS & Emission Factor, $\mu \mathrm{g} / \mathrm{cig}$ & Comments \\
\hline Benzene & This study & $\begin{array}{c}\text { ETS } \\
\text { SS }\end{array}$ & $\begin{array}{l}406 \pm 71 \\
248 \pm 70\end{array}$ & \\
\hline & Jermini, et al., 1976 & ETS & 415 & 30 American cigareltes smoked simultaneously in $30-\mathrm{m}^{3}$ chamber \\
\hline & Lofroth, et al., 1989 & ETS & 500 & $\begin{array}{l}13.6-\mathrm{m}^{3} \text { chamber with continuous smoking over sampling period, } 1 \\
\mathrm{cig} / 15 \text { minutes or } 1 \mathrm{cig} / 30 \text { minutes }\end{array}$ \\
\hline & Brunnemann, et al., 1990 & ETS & $496^{\mathrm{b}}$ & $\begin{array}{l}16-\mathrm{m}^{3} \text { unventilated chamber; } 5 \text { cigarettes smoked; emission factor } \\
\text { estimated from concentration, assuming air exchange rate of zero } \\
\text { SS generated in glass chamber at airflow rate of } 1.5 \mathrm{~L} \mathrm{~min}{ }^{-1} \text {; SS } \\
\text { filtered and gases trapped in methanol at }-78^{\circ} \mathrm{C}\end{array}$ \\
\hline 1,3- Butadiene & This study & $\begin{array}{l}\text { ETS } \\
\text { SS }\end{array}$ & $\begin{array}{c}152 \pm 27 \\
\text { Below detection }\end{array}$ & \\
\hline & Lofroth, et al., 1989 & ETS & 400 & $\begin{array}{l}13.6-\mathrm{m}^{3} \text { chamber with continuous smoking over sampling period, } 1 \\
\text { cig } / 15 \text { minutes or } 1 \text { cig/30 minutes }\end{array}$ \\
\hline & Brunnemann, et al., 1990 & ETS & 30 & $\begin{array}{l}16-\mathrm{m}^{3} \text { unventilated chamber; } 5 \text { cigarettes smoked; emission factor } \\
\text { estimated from concentration, assuming air exchange rate of zero } \\
\text { SS generated in glass chamber at airflow rate of } 1.5 \mathrm{~L} \mathrm{~min}{ }^{-1} ; \mathrm{SS} \\
\text { filtered and gases trapped in methanol at }-78^{\circ} \mathrm{C}\end{array}$ \\
\hline 2-Butanone & This study & $\begin{array}{c}\text { ETS } \\
\text { SS }\end{array}$ & $\begin{array}{l}291 \pm 56 \\
197 \pm 52 \\
\end{array}$ & \\
\hline & Jermini, et al., 1976 & ETS & 804 & 30 American cigarettes smoked simultaneously in $30-\mathrm{m}^{3}$ chamber \\
\hline
\end{tabular}

a. Average \pm Std. Dev. for six commercial brands.

b. Concentrations for benzene and toluene appear to have been reversed in Table VI of Brunnemann et al., 1990; we have taken toluene as benzene and vice-versa. 
Table 6.16, Continued. Comparison of the VOC emission factors determined in this study ${ }^{\text {a. }}$ to literature data.

\begin{tabular}{|c|c|c|c|c|}
\hline Compound & Study & ETS or SS & Emission Factor, $\mu \mathrm{g} / \mathrm{cig}$ & Comments \\
\hline \multirow[t]{3}{*}{ m,p-Cresol } & This study & $\begin{array}{c}\text { ETS } \\
\text { SS }\end{array}$ & $\begin{array}{c}83 \pm 26 \\
\text { Below detection }\end{array}$ & \\
\hline & $\begin{array}{l}\text { Chortyk and Schlotzhauer, } \\
1989\end{array}$ & SS & $\begin{array}{c}132 \pm 53 \\
\text { Range: } 59-299\end{array}$ & $\begin{array}{l}\text { SS smoke condensates from } 10 \text { cigarettes collected in solvent trap } \\
\text { containing 3:1 acetone/chloroform; } 20 \text { low tar American cigarettes }\end{array}$ \\
\hline & $\begin{array}{l}\text { Eatough } \text { et al., } 1990- \\
\text { Summary of literature data }\end{array}$ & SS & $42-93$ & Variety of methods and brands \\
\hline \multirow[t]{3}{*}{ o-Cresol } & This study & $\begin{array}{l}\text { ETS } \\
\text { SS }\end{array}$ & $\begin{array}{c}35 \pm 5 \\
\text { Below detection }\end{array}$ & \\
\hline & $\begin{array}{l}\text { Chortyk and Schlotzhauer, } \\
1989\end{array}$ & SS & $\begin{array}{c}58 \pm 17 \\
\text { Range: } 24-98\end{array}$ & $\begin{array}{l}\text { SS smoke condensates from 10 cigarettes collected in solvent trap } \\
\text { containing 3:1 acetone/chloroform; } 20 \text { low tar American cigarettes }\end{array}$ \\
\hline & \begin{tabular}{|l|} 
Eatough et al., $1990-$ \\
Summary of literature data
\end{tabular} & SS & $14-18$ & Variety of methods and brands \\
\hline \multirow[t]{3}{*}{ Phenol } & This study & $\begin{array}{c}\text { ETS } \\
\text { SS }\end{array}$ & $\begin{array}{c}281 \pm 61 \\
\text { Below detection }\end{array}$ & \\
\hline & $\begin{array}{l}\text { Chortyk and Schlotzhauer, } \\
1989\end{array}$ & SS & 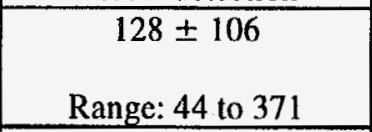 & $\begin{array}{l}\text { SS smoke condensates from } 10 \text { cigarettes collected in solvent trap } \\
\text { containing } 3: 1 \text { acetone/chloroform; } 20 \text { low tar American cigarettes }\end{array}$ \\
\hline & $\begin{array}{l}\text { Eatough } \text { et al., } 1990 \text { - } \\
\text { Summary of literature data }\end{array}$ & $\overline{\text { SS }}$ & $52-290$ & Variety of methods and brands \\
\hline \multirow[t]{2}{*}{ Pyridine } & This study & $\begin{array}{c}\text { ETS } \\
\text { SS }\end{array}$ & $\begin{array}{c}428 \pm 122 \\
394 \pm 80\end{array}$ & \\
\hline & $\begin{array}{l}\text { Eatough et al., } 1990 \text { - } \\
\text { Summary of literature data }\end{array}$ & SS & $160-3400$ & Variety of methods and brands \\
\hline \multirow[t]{2}{*}{ Pyrrole } & This study & $\begin{array}{l}\text { ETS } \\
\text { SS }\end{array}$ & $\begin{array}{l}402 \pm 90 \\
411 \pm 87 \\
\end{array}$ & \\
\hline & $\begin{array}{l}\text { Eatough et al., } 1990- \\
\text { Summary of literature data }\end{array}$ & SS & $140-270$ & Variety of methods and brands \\
\hline \multirow[t]{2}{*}{ Styrene } & This study & $\begin{array}{l}\text { ETS } \\
\text { SS }\end{array}$ & $\begin{array}{l}147 \pm 24 \\
102 \pm 22\end{array}$ & \\
\hline & Jermini, et al., 1976 & ETS & 98 & 30 American cigarettes smoked simultaneously in $30-\mathrm{m}^{3}$ chamber \\
\hline
\end{tabular}

a. Average \pm Std. Dev. for six commercial brands.

b. Concentrations for benzene and toluene appear to have been reversed in Table VI of Brunnemann et al., 1990; we have taken toluene as benzene and vice-versa. 
Table 6.16, Continued. Comparison of the VOC emission factors determined in this study ${ }^{\text {a. }}$ to literature data.

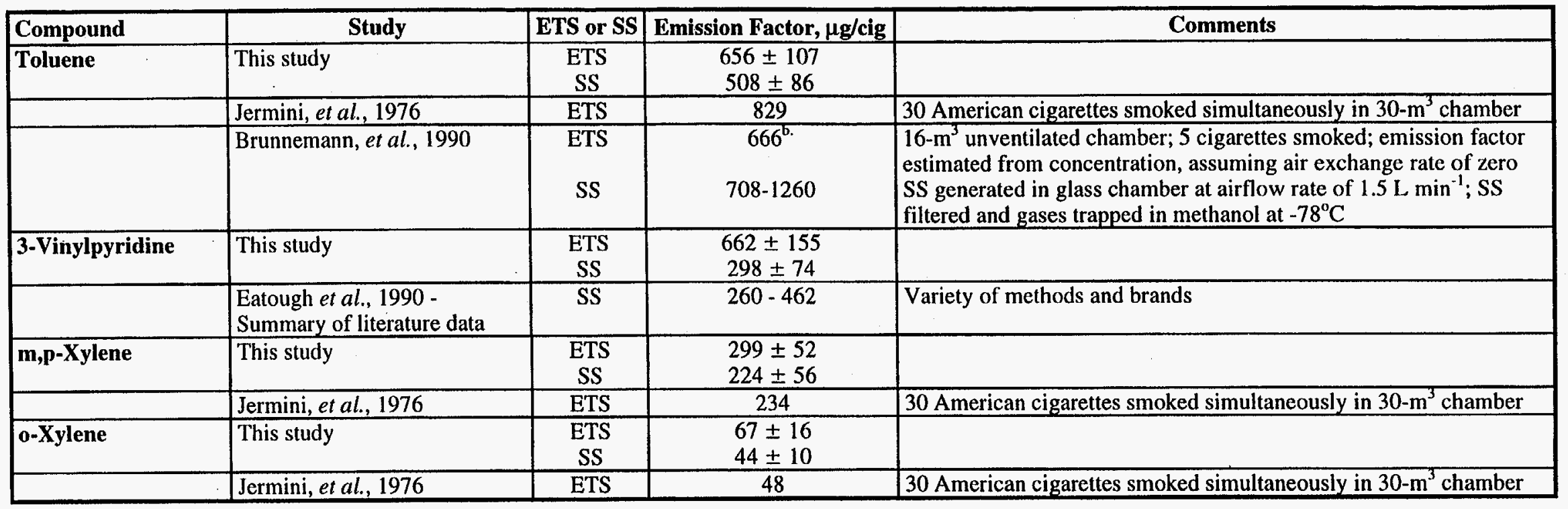

a. Average \pm Std. Dev. for six commercial brands.

b. Concentrations for benzene and toluene appear to have been reversed in Table VI of Brunnemann et al., 1990; we have taken toluene as benzene and vice-versa. 
In our chamber measurements of ETS, we did not see consistent decreases in chamber air concentrations of 1,3-butadiene over the four-hour measurement period, within the experimental uncertainties of sampling and analysis $(\sim 25 \%)$. We hypothesize that the major difference between the ETS emission factors from the Brunnemann, et al. (1990) experiments and from our experiments is related to differences in the interior surface materials of the two chambers. It is possible that the low emission factors from their measurements were due to chemical reactions with surface materials which presumably were typical of surfaces in buildings, (e.g., painted wallboard or plaster walls). The chemical reactivities for typical surfaces are expected to be quite different from those of stainless steel.

In general, the ETS emission factors for all of the VOCs in Table 6.16 except 1,3-butadiene are in surprisingly good agreement among various investigators. The VOCs which are relatively stable chemically and showed no evidence of deposition to surfaces (benzene, toluene, styrene and xylene isomers) are in very good agreement. Even for those compounds for which there is evidence of deposition losses in the chamber, the emission factors reported by various investigators generally agree within a factor of two.

In summary, the ETS emission factors reported in Table 6.9, with the exception of the factor for 1,3-butadiene, are recommended for use in estimating Californian's indoor exposures to those compounds. The ETS emission factors reported in Tables 6.11 and 6.12 for the VOCs which showed evidence of depositional losses to surfaces may also be used but with the understanding that they will provide "upper limit" estimates unless corrections are made for such losses. At present, we do not have the information that would allow us to correct for these losses in "real world" settings. The deposition rate constants reported in Table 6.10 apply only to the stainless steel chamber and cannot yet be generalized to buildings. Re-emission of sorbed VOCs is also possible but cannot be taken into account in indoor models due to the lack of compound-specific information and a theoretical basis for generalizing sorption and desorption processes for environments with different materials and air flow characteristics.

In the case of 1,3-butadiene, we suspect that there may be chemical reactions that occur rapidly in the initial generation of ETS as well as subsequent chemical reactions with "real world" surface materials.

\subsubsection{Nicotine}

Indoor measurements of vapor-phase nicotine have been widely used to trace exposures to ETS. Nicotine meets many of the criteria for an ETS tracer. It is unique to tobacco; it is emitted in relatively large amounts; it is easily measured in indoor air; and its ETS emission factors are very similar among brands of cigarettes (Leader and Hammond, 1991). In addition, the ratios of many components of ETS to nicotine are similar among brands (U.S. EPA, 1992). In room-size chambers, $90-95 \%$ of the nicotine is

found in the vapor-phase. However, airborne nicotine concentrations typically decrease with time due to 
deposition of nicotine on indoor surfaces (e.g., U.S. EPA, 1992; Nelson, et al., 1992; Eatough, 1993). The deposition, sorption and re-emission of nicotine from indoor sources is quite complex and study of those processes was beyond the scope of this project. Nevertheless, field studies have demonstrated the usefulness of nicotine concentrations for estimating indoor exposures to ETS (Hammond, et al., 1987; Leader and Hammond, 1991; U.S. EPA, 1992). Therefore, we measured average nicotine concentrations in the environmental chamber over the four-hour experimental period.

SS emission factors for nicotine were also measured for completeness. The phase distribution of nicotine, however, is dependent upon the size and surface-to-volume ratio of the chamber in which such measurements are made. During method development, we observed only $5 \%$ of the nicotine in the XAD-4 sorbent bed downstream to the filter; this represents the vapor-phase fraction of nicotine in SS. The remainder was condensed in the SSA or trapped on the filter. Therefore, the SS nicotine emission factors reported here are the total amounts of nicotine emitted as sidestream smoke. The ETS emission factors however, are calculated from the time-averaged vapor-phase nicotine concentrations in the chamber.

Reproducibility of emission measurements. Table 6.17 summarizes the ETS and SS emission factors for the six commercial brands of cigarettes and reference cigarette 1R4F. Duplicate determinations were made of the ETS emission factors for brand $A$ and cigarette 1R4F and of the SS emission factor for brand $\mathrm{A}$. The deviations of the duplicate values from the means were less than $4 \%$ of the mean. The estimated uncertainty associated with the sampling and analysis of nicotine was about $8 \%$ (Tables 5.10 and 5.11). Thus, the reproducibility of the ETS and SS emission factors was very good, and any variability introduced by the chamber was insignificant.

Variability of the ETS emission factors among cigarette brands. The variability of the ETS nicotine emission factors, expressed as $\mathrm{ng} / \mathrm{mg}$ tobacco, among the six commercial brands of cigarettes was $18 \%$, as measured by the coefficient of variation of the mean (Table 6.17). The ratio of the highest to the lowest nicotine emission factors (ng/mg) was 1.5. For the emission factors expressed as $\mu \mathrm{g} / \mathrm{cig}$, the coefficient of variation was larger at $26 \%$. The ratio of the highest to the lowest nicotine emission factor $(\mu \mathrm{g} / \mathrm{cig})$ was 2.0 . The emission factors for the reference cigarette fell within the ranges of the emission factors for the commercial cigarettes. The variability of the nicotine ETS emission factors among brands was only about twice the estimated variability due to sampling and analysis.

Variability of SS emission factors among cigarette brands. The variability of the SS emission factors, expressed as ng/mg tobacco, among brands of cigarettes was only $1 \%$ (Table 6.17). For the emission factors expressed as $\mu \mathrm{g} / \mathrm{cig}$, the coefficient of variation was $15 \%$. 
Table 6.17. Summary of nicotine emission factors for environmental tobacco smoke and sidestream smoke from six commercial cigarettes and Kentucky reference cigarette 1R4F.

\begin{tabular}{|c|c|c|c|c|}
\hline Cigarette & $\begin{array}{c}\text { ETS, } \\
\text { ng/mg }\end{array}$ & $\begin{array}{c}\mathrm{SS}, \\
\mathrm{ng}^{\prime} \mathrm{mg}^{\mathrm{b}}\end{array}$ & $\begin{array}{c}\text { ETS, } \\
\mu \mathrm{g} / \mathrm{cig}^{\mathrm{c}}\end{array}$ & $\begin{array}{c}\text { SS, } \\
\mu \mathrm{g} / \mathrm{cig}^{\mathrm{d}}\end{array}$ \\
\hline $\mathbf{A}$ & 1,700 & 7,940 & 1,040 & 4,860 \\
\hline $\mathrm{A}^{\mathrm{e}}$ & 1,810 & 7,960 & 1,110 & 4,870 \\
\hline B & 1,250 & 7,850 & 709 & 4,460 \\
\hline C & 1,250 & 7,740 & 662 & 4,120 \\
\hline D & 1,200 & 7,960 & 767 & 5,070 \\
\hline $\mathbf{E}$ & 1,730 & 7,870 & 1,350 & 6,160 \\
\hline $\mathbf{F}$ & 1,260 & 7,870 & 913 & 5,690 \\
\hline 1R4F & 1,480 & 8,250 & 956 & 5,350 \\
\hline $1 R 4 F^{e}$ & 1,590 & - & 1,030 & - \\
\hline Average $e^{f}$ & 1,410 & 7,860 & 919 & 5,060 \\
\hline Std. Dev." & 260 & 82 & 240 & 763 \\
\hline C. V., g. & 18 & 1 & 26 & 15 \\
\hline
\end{tabular}

a. $\mathrm{ng} / \mathrm{mg}=\mathrm{ng}$ of nicotine emitted by one $\mathrm{mg}$ of cigarette tobacco.

b. $\mathrm{ng} / \mathrm{mg}=$ Total $\mathrm{ng}$ of nicotine (sorbent tube and apparatus) emitted by one $\mathrm{mg}$ of cigarette tobacco.

c. $\mu \mathrm{g} / \mathrm{cig}=\mathrm{ng}$ of nicotine $\mathrm{x}$ standard length / actual smoked length.

d. $\mu \mathrm{g} / \mathrm{cig}=$ Total $\mathrm{ng}$ of nicotine (sorbent tube and apparatus) $\mathrm{x}$ standard length $/$ actual smoked length.

e. Duplicate experiment.

f. 1 R4F omitted; $n=6$.

g. C. V.: Coefficient of Variation $=($ Std. Dev. X 100) /Average.

Comparison of ETS and SS emission factors. The ETS nicotine emission factors were only 15 to $22 \%$ of the SS emission factors, and the difference between the two means was statistically significant $(p<0.001)$. Therefore, only about one-fifth of the total nicotine emitted by the cigarettes persisted in chamber air over the duration of the experiment. As previously discussed, much of the SS nicotine is rapidly deposited onto surfaces. The difference between our ETS and SS measurements is presumably due to deposition losses in the chamber and the fact that total nicotine was measured for SS. Deposition losses in buildings would be expected to be even higher. Deposited nicotine can also be re-emitted into air.

Comparison to previously reported emission factors. Table 6.18 summarizes some of the more recent measurements of nicotine ETS and SS emission factors reported in the literature. In many of the studies, cigarettes were machine-smoked using the same protocol as in our study. The reported emission factors depend upon the chamber type and its operation and on the selection of cigarettes. Our SS emission factor for reference cigarette $1 \mathrm{R} 4 \mathrm{~F}$ of $5,350 \mu \mathrm{g}$ per cigarette is in excellent agreement with the nicotine emission factor published by R. J Reynolds (1988) of 5,600 $\mu \mathrm{g}$ per cigarette for the same reference cigarette. 
Hammond, et al. (1987) measured emission factors for four cigarette brands (two high tar and two low tar) in a 34- $\mathrm{m}^{3}$ aluminum chamber operated at an air exchange rate of $2.4 \mathrm{~h}^{-1}$. Air in the chamber was recirculated at a rate of $95 \mathrm{~h}^{-1}$ for mixing. In these experiments, four smokers smoked eight cigarettes per hour for five hours. Sampling was conducted after chamber concentrations of particles had reached steady state. ETS (diluted SS plus exhaled mainstream smoke) emission factors for nicotine ranged from 870 to 1,130 with an average of $970 \pm 140 \mu \mathrm{g}$ per cigarette.

In a later study, Leaderer and Hammond (1991) compared ratios of particles to nicotine for 10 U.S. cigarette brands. The brands were selected to cover a range of high to low tar and nicotine (mainstream values). Chamber protocols were identical to those in the earlier study (Hammond, et al., 1987). During these experiments, concentrations of RSP ranged from 953 to $1,789 \mu \mathrm{g} / \mathrm{m}^{3}$, and nicotine concentrations ranged from 70 to $120 \mu \mathrm{g} / \mathrm{m}^{3}$. We calculated nicotine ETS emission factors.from their data. They varied from 850 to 1,470 with an average of $1,230 \pm 190 \mu \mathrm{g}$ per cigarette. The nicotine ETS emission factors in our study varied over a similar range with an average of $919 \pm 240 \mu \mathrm{g}$ per cigarette and were, therefore, in good agreement with the other values despite differences in cigarettes and chambers.

Lofroth, et al. (1989) conducted experiments in a $13.6-\mathrm{m}^{3}$ Plexiglas chamber. In the first series of experiments, in which the chamber was occupied by two persons and contained some furnishings, the nicotine ETS emission factor was $800 \mu \mathrm{g} / \mathrm{cig}$. In the second series, in which the chamber was unoccupied, the average nicotine emission factor was $3,000 \mu \mathrm{g} / \mathrm{cig}$. Additional depositional losses to occupants and furnishings was suggested as the explanation for the difference. Their lower value falls within the range of factors measured in the present study and in the studies by Hammond, et al. (1987) and Leaderer and Hammond, (1991).

The SS nicotine emission factors reported in Table 6.18 are all much higher than the ETS emission factors, as might be expected. The presented values are also reasonably consistent despite differences in the cigarettes selected for each study. Guerin, et al. (1987) reviewed older published studies of SS emission factors for nicotine and reported a range of $2,700-6,900 \mu \mathrm{g}$ per cigarette.

In summary, both our ETS and SS emission factors for nicotine are in good agreement with literature values. The large difference we observed between the ETS and SS emission factor (factor of five)' is also consistent with the literature. The SS emission factors are the total amounts of nicotine emitted as sidestream smoke; whereas, only vapor-phase concentrations of nicotine were measured in the chamber. Nicotine has a low vapor pressure and, therefore, a high tendency for deposition on surfaces. We did not measure the decay of nicotine in the chamber due to deposition losses as only a single time-weighted 
Table 6.18. Comparison of nicotine emission factors determined in this study to literature data.

\begin{tabular}{|c|c|c|c|c|}
\hline \multirow[t]{2}{*}{ Study } & \multirow[t]{2}{*}{$\begin{array}{l}\text { ETS } \\
\text { or SS } \\
\end{array}$} & \multicolumn{2}{|c|}{$\begin{array}{c}\text { Emission Factor, } \\
\mu \mathrm{g} / \mathrm{cig}\end{array}$} & \multirow[t]{2}{*}{ Comments } \\
\hline & & Mean \pm S.D. & Range & \\
\hline This Study & ETS & $920 \pm 240$ & $710-1,350$ & $\begin{array}{l}6 \text { American brands }(62 \% \text { of } \\
\text { California market }) ; \quad 20-\mathrm{m}^{3} \\
\text { chamber, } 0.03 \text { ach; } 3 \text { cigarettes } \\
\text { smoked at start }\end{array}$ \\
\hline Hammond, et al., 1987 & ETS & $1,240 \pm 180$ & $1,070-1,450$ & $\begin{array}{l}\text { Two high and two low nicotine } \\
\text { cigarette brands; } 34-\mathrm{m}^{3} \text { aluminum } \\
\text { chamber, } 2.5 \text { ach with } \\
\text { recirculation (mixing) set at } 95 \mathrm{~h}^{\circ} \\
1,8 \text { cigarettes } / \mathrm{hr} \text { smoked over } 5 \\
\text { hours. }\end{array}$ \\
\hline $\begin{array}{l}\text { Leaderer and Hammond, } \\
1991\end{array}$ & ETS & $1,230 \pm 200$ & $850-1,470$ & $\begin{array}{l}10 \text { American brands; } 34-\mathrm{m}^{3} \\
\text { aluminum chamber, } 2.5 \text { ach with } \\
\text { recirculation (mixing) set at } 95 \mathrm{~h}^{-} \\
1,8 \text { cigarettes } / \mathrm{hr} \text { smoked over } 5 \\
\text { hours. }\end{array}$ \\
\hline Lofroth, et al., 1989 & ETS & - & $800,3,000$ & $\begin{array}{l}\text { One } 2 \mathrm{R} 1 \text { cigarette smoked per } 15 \\
\text { or } 30 \text { minutes in a 13.6- } \mathrm{m}^{3} \\
\text { Plexiglas chamber, } 4 \text { ach; } 4 \\
\text { people in chamber in first } \\
\text { experiment, none in second }\end{array}$ \\
\hline This study & SS & $5,060 \pm 760$ & $4,100-5,700$ & 6 American brands \\
\hline Rickert, et al., 1984 & SS & $4,100 \pm 1,000$ & $2,700-6,100$ & 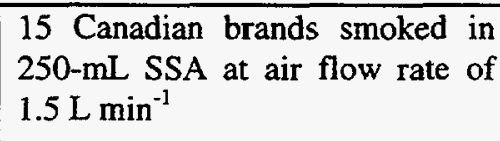 \\
\hline $\begin{array}{l}\text { Chortyk and } \\
\text { Schlotzhauer, } 1987\end{array}$ & SS & $8,040 \pm 1,380$ & $\begin{array}{l}5,700- \\
10,500\end{array}$ & 20 Low tar American brands \\
\hline R.J. Reynolds, 1988 & SS & 5,600 & - & 1R4F reference cigarette \\
\hline
\end{tabular}

average sample was collected. Nevertheless, the losses are expected to be relatively high. Consequently, nicotine is not an ideal tracer for vapor-phase compounds in ETS.

\subsubsection{Particulate Matter}

Although the focus of this study was on the determination of emission factors for vapor-phase air toxics, ETS particulate matter emission factors were also measured during the chamber experiments. This was done to: 1) provide a more complete set of emission factors; 2) examine the ratio of particulate matter to nicotine for comparison to earlier studies; and 3) provide ratios of particulate matter to nicotine that could be used to predict concentrations of ETS derived particulate matter from measured nicotine concentrations. 
Samples of ETS particulate matter were collected on filters, without a cyclone pre-filter to remove particles greater than $2.5 \mu \mathrm{m}\left(\mathrm{D}_{50}\right)$ because experimental work at LBL (Offermann, et al., 1985; Xu, et al., 1994) has demonstrated that the mass median particle diameter of ETS is about $0.2 \mu \mathrm{m}$, with a $\sigma_{\mathrm{g}}$ of about 2. Based on these studies, $98 \%$ of the particles collected in the chamber would be less than $0.8 \mu \mathrm{m}$ diameter. Thus, the samples were equivalent to those which would have been measured with a PM-2.5 sampler with a cyclone pre-filter and are designated as PM-2.5 $5_{\mathrm{EQ}}$.

The ETS emission factors were corrected for deposition losses to chamber surfaces as described in Section 5.8.1. These corrected values are presented in Table 6.19. For comparison, the corresponding uncorrected values are also shown. Particulate matter was measured in only one of the duplicate experiments with cigarette A, therefore, the reproducibility of the ETS measurements can not be assessed.

Variability among cigarette brands. The average corrected emission factor, expressed as $\mu \mathrm{g}$ of ETS PM-2.5 $5_{\mathrm{EQ}}$ per mg of tobacco was $12.4 \pm 1.3$ for the six commercial cigarette brands (Table 6.19 ). The variability among brands was $11 \%$, and the ratio of the highest to lowest emission factor was 1.3 . The average corrected ETS PM-2.5 $5_{\mathrm{EQ}}$ emission factor, expressed as mg/cig, was $8.1 \pm 2.0$, and the coefficient of variation was $25 \%$ among the brands. The ratio of the highest to the lowest emission factor was 1.8 . The corresponding uncorrected values are lower by a factor of 0.86 .

Table 6.19 also lists the tar values for mainstream smoke that have been reported by the FTC (1992). These were examined to determine if there was any correlation between tar values and the ETS particulate matter emission factors. However, the value of $r^{2}$ for the correlation was only 0.04 , indicating that there is no significant relationship between ETS particulate emissions and the mainstream tar values. Mainstream emissions are highly dependent upon the presence and design of filters which remove much of the tar, and these filters have little or no effect on SS and ETS emissions.

SS particulate emission factors. The total SS particulate mass, defined as the mass collected onto the TCGF filter plus the mass collected from the SSA condensate, is reported for each cigarette brand in Table 6.20. The filters were observed to have collected some water. No attempt was made to correct for this artifact. The average emission factor for SS particulate mass was $30 \mathrm{mg}$ per cigarette, and the variation among the brands was $19 \%$. The SS values are about four times the ETS values. As noted here and as discussed in Section 5.7.4, there are substantial artifact problems associated with measuring emission factors for SS particulate matter due to condensation on the SSA and the filter. Therefore, the SS values can not reliably be used to estimate concentrations of airborne particulate matter that would be found in the air of a room due to smoking. 
Table 6.19. Summary of corrected and uncorrected ETS emission factors for PM-2.5 $5_{\text {EQ }}$ for six commercial cigarettes and Kentucky reference cigarette 1R4F.

\begin{tabular}{|c|c|c|c|c|c|}
\hline \multirow[b]{2}{*}{ Cigarette } & \multicolumn{2}{|c|}{ Corrected PM-2.5 } & \multicolumn{2}{|c|}{ Uncorrected PM-2.5 $5_{\mathrm{E} O}$} & \multirow{2}{*}{$\begin{array}{c}\text { FTC Tar } \\
\text { Values } \\
\text { mg/cig } \\
\end{array}$} \\
\hline & $\mu \mathrm{g} / \mathbf{m g}^{2}$ & mg/cig ${ }^{\text {b. }}$ & $\mu \mathrm{g} / \mathrm{mg}^{\mathrm{a}}$ & $\mathrm{mg} / \mathrm{cig}^{\mathrm{b}}$ & \\
\hline $\mathbf{A}$ & 12.8 & 7.8 & 11.1 & 6.8 & 16 \\
\hline B & 11.8 & 6.7 & 10.1 & 5.8 & 11 \\
\hline $\mathbf{C}$ & 11.0 & 5.9 & 9.5 & 5.1 & 22 \\
\hline $\mathbf{D}$ & 11.1 & 7.1 & 9.6 & 6.1 & 16 \\
\hline $\mathbf{E}$ & 13.9 & 10.9 & 12.0 & 9.4 & 15 \\
\hline $\mathbf{F}$ & 13.9 & 10.1 & 12.0 & 8.7 & 17 \\
\hline 1R4F & 11.9 & 7.7 & 10.2 & 6.6 & - \\
\hline Average $^{\mathrm{d}}$ & 12.4 & 8.1 & 10.7 & 7.0 & \\
\hline Std. Dev. & 1.3 & 2.0 & 1.1 & 1.7 & \\
\hline C. V.e. & 11 & 25 & 11 & 25 & \\
\hline
\end{tabular}

a. Average particulate matter emission factor in $\mu \mathrm{g}$ per $\mathrm{mg}$ of tobacco consumed for 250 -minute sampling period.

b. Average particulate matter emission factor in $\mathrm{mg}$ per cigarette for 250 -minute sampling period.

c. Values reported by FTC (1992) for mainstream smoke.

d. IR4F omitted; $\mathrm{n}=6$.

e. C. V.: Coefficient of Variation $=($ Std. Dev. X 100)/Average.

Table 6.20. Summary of SS particulate matter emission factors for six commercial cigarettes and Kentucky reference cigarette 1R4F.

\begin{tabular}{|c|c|c|c|c|}
\hline Cigarette & $\begin{array}{l}\text { TCGF }^{\text {a }} \text {, } \\
\text { mg/cig }\end{array}$ & $\begin{array}{l}\mathrm{SSA}^{\mathrm{b}} \text {, } \\
\mathrm{mg} / \mathrm{cig}\end{array}$ & $\begin{array}{l}\text { Total } \\
\text { mg/cig }\end{array}$ & $\begin{array}{l}\text { Total }^{\mathrm{d}} \text {, } \\
\mu \mathrm{g} / \mathrm{mg}\end{array}$ \\
\hline $\mathbf{A}$ & 23 & 5.9 & 29 & 47 \\
\hline $\mathbf{A}^{\text {e. }}$ & 25 & 6.2 & 32 & 54 \\
\hline B & 22 & 4.8 & 26 & 46 \\
\hline $\mathrm{C}$ & 19 & 4.8 & 24 & 45 \\
\hline $\bar{D}$ & 21 & 5.8 & 27 & 42 \\
\hline $\mathbf{E}$ & 33 & 6.5 & 40 & 51 \\
\hline $\mathbf{F}$ & 28 & 5.7 & 34 & 47 \\
\hline 1R4F & 27 & 4.4 & 32 & 49 \\
\hline Average $^{\text {f. }}$ & 24 & 5.6 & 30 & 47 \\
\hline Std. Dev. & 5.2 & 0.7 & 5.7 & 3.3 \\
\hline C. V.g. & 21 & 12 & 19 & 7 \\
\hline
\end{tabular}
a. Particulate mass collected on the Teflon-coated glass-fiber filter during SS sampling.
b. Particulate mass condensed onto inner walls of the sidestream smoke apparatus.
c. Total particulate mass [TCGF filter + SSA condensate] found in SS.
d. Total particulate mass collected onto filter and SSA wall per mg of tobacco consumed.
e. Duplicate experiment.
f. 1R4F omitted; $n=6$
g. C. V.: Coefficient of Variation $=($ Std. Dev. X 100)/Average. 
Comparison of emission factors determined in environmental chambers. There have been several recent reports of environmental chamber measurements of particulate matter emission factors. These are summarized and compared in Table 6.21. The particulate emission factors from the four studies are in quite good agreement despite differences in the cigarette brands and the experimental protocols.

The averages for the studies by Hammond, et al. (1987) and Leaderer and Hammond (1991), are about 60 to $79 \%$ higher than the average we measured. This difference may be due in part to the differences in the smoking protocols. In their studies, cigarettes were continuously smoked by human subjects during sampling to achieve steady-state concentrations of particulate matter which included the exhaled portion of mainstream smoke. In our experiments, three cigarettes were machine smoked and only the sidestream smoke was emitted into the chamber. Sample collection did not begin until all three cigarettes were smoked. Therefore, our sampled ETS particles were more "aged" and did not include any exhaled mainstream component. Baker and Proctor (1990) have reported that exhaled MS contributes about $15 \%$ of the total particulate matter in ETS. Benner et al. (1989) reported results of experiments which demonstrated volatilization losses from ETS particles over time in a sealed $30-\mathrm{m}^{3}$ Teflon chamber. They measured decreases in ETS particle mass concentrations, changes in particle-size distributions and increases in total vapor-phase hydrocarbons over time and took particle coagulation and wall losses of particles into account. Their results showed that particle mass shifted to the vapor phase as the aerosol aged. In summary, the measured ETS emission factors in this study are in good agreement with recently reported data when differences in smoking protocols are taken into account.

Comparison of ETS PM-2.5 to ETS Nicotine Ratios. The ratio of PM-2.5 to nicotine concentrations has been measured in environmental chambers and in a field study of residences (Leaderer and Hammond, 1991; U.S. EPA, 1992). This ratio is of interest because it can be used to estimate how much of the particulate mass concentration in a building is from ETS. In this "chemical marker" approach, the measured concentration of nicotine in a building is assumed to come only from ETS and is multiplied by the ratio of particulate matter to nicotine in ETS to obtain the concentration of particles that originates from ETS. Table 6.22 compares measurements of this ratio determined in chamber studies, and in a field study.

In the environmental chamber experiments conducted by Leaderer and Hammond (1991), a ratio of $14 \pm 2$ was obtained for 10 cigarette brands. In this same study, the investigators measured respirable suspended particulate matter (RSP), i.e., ETS RSP and background RSP, and nicotine in 96 residences in New York state over one-week periods. Forty-seven of the residences had smokers. The plots of RSP versus nicotine had slopes (RSP/nicotine ratio) of 10.8 for all 96 houses and 9.8 for the 47 homes with smokers. These ratios are similar to those obtained in chamber studies. The intercepts of the lines were interpreted as particulate matter from outdoor and any other indoor sources. 
Table 6.21. ETS particulate matter emission factors determined in environmental chambers.

\begin{tabular}{|c|c|c|}
\hline Investigation & $\begin{array}{l}\text { Average ETS } \\
\text { Emission } \\
\text { Factor, } \mathrm{mg} / \text { cig }\end{array}$ & Comments \\
\hline This Study & $8.1 \pm 2.0$ & $\begin{array}{l}\text { Six commercial brands ( } 62 \% \text { of California market); } \\
3 \text { cigarettes machine-smoked in } 20-\mathrm{m}^{3} \text { chamber } \\
\text { followed by sample collection over a } 4 \text {-hour period. } \\
\text { PM-2.5 } 5_{\mathrm{EQ}}: 500-1300 \mu \mathrm{g}-\mathrm{m}^{-3}\end{array}$ \\
\hline Lofroth, et al., 1989 & 10 & $\begin{array}{l}2 \mathrm{R} 1 \text { cigarettes smoked by smokers in } 13.6-\mathrm{m}^{3} \\
\text { Plexiglas chamber, } 4.05 \mathrm{~h}^{-1} \text { air exchange rate; } 1 \\
\text { cigarette smoked every } 15 \text { or } 30 \text { minutes throughout } \\
\text { the experiments; average of } 13 \text { experiments } \\
\text { reported. PM-2.5 } 5_{\mathrm{EQ}}: 349 \pm 39,321 \pm 25,934 \pm 46 \\
\mu \mathrm{g}-\mathrm{m}^{-3}\end{array}$ \\
\hline Hammond, et al., 1987 & $13.0 \pm 1.2$ & $\begin{array}{l}2 \text { high and } 2 \text { low tar brands; } 8 \text { cigarettes per hour } \\
\text { smoked by } 4 \mathrm{smokers} \mathrm{in} \mathrm{a} 34-\mathrm{m}^{3} \text { chamber; air } \\
\text { exchange rate } 2.5 \mathrm{~h}^{-1} \text {, recirculation rate } 95-\mathrm{h}^{-1}\end{array}$ \\
\hline Leaderer and Hammond, 1991 & $14.5 \pm 2.0$ & $\begin{array}{l}\text { Average for } 10 \text { brands of American cigarettes, } \\
\text { ranging from high to low tar; chamber and } \\
\text { protocols were the same as for Hammond, et al., } \\
\text { 1987. RSP concentrations: } 953-1789 \mu \mathrm{g}-\mathrm{m}^{-3}\end{array}$ \\
\hline
\end{tabular}

Table 6.22. Comparison of ratios of ETS particulate matter to ETS nicotine.

\begin{tabular}{|l|c|l|}
\hline Study & $\begin{array}{c}\text { Ratio of } \\
\text { particulate matter to nicotine }\end{array}$ & \multicolumn{1}{c|}{ Comments } \\
\hline This study & $\begin{array}{c}9.0 \pm 1.3 \\
(7.2 \text { to } 11.1)\end{array}$ & $\begin{array}{l}\text { Chamber study; 6 American } \\
\text { brands }\end{array}$ \\
\hline Hammond, et al., 1987 & 11.8 to 14.5 & $\begin{array}{l}\text { Chamber study; 4 American } \\
\text { brands, 2 high and 2 low tar }\end{array}$ \\
\hline Lofroth, et al., 1989 & $12 \pm 3^{\mathrm{a} .}$ & Chamber study; 2R1 cigarettes \\
\hline Leaderer and Hammond, 1991 & $14 \pm 2$ & $\begin{array}{l}\text { Chamber study; 10 American } \\
\text { brands }\end{array}$ \\
\hline Leaderer and Hammond, 1991 & $10.8 \pm 0.72^{\mathrm{b} ., \mathrm{c}}$ & $\begin{array}{l}\text { Measurements in 96 homes, 47 } \\
\text { with smokers }\end{array}$ \\
\hline
\end{tabular}

a. RSP/nicotine; variability estimated from standard deviations of means of RSP and nicotine.

b. Slope \pm standard error of the slope from a regression analysis of RSP versus nicotine. The intercept of the line was $17.9 \pm 1.6$ (S.E.) and was interpreted as particulate matter from outdoor air plus any other indoor sources.

c. The standard error of the slope and intercept were not reported in the original publication but in U.S. EPA (1992). 
There are other published data that can be used to calculate the ratio of particulate matter to nicotine. For example, Meisner et al. (1989) measured PM-2.5 particulate matter and nicotine in a variety of public facilities and office buildings. Overall, the ratio of particulate concentration to nicotine concentration was about 15 with no corrections for background concentrations of particles. When the points with the two highest nicotine concentrations were removed from the data set, the ratio decreased to 9 . Nagda et al. (1992) measured concentrations of RSP and nicotine in 92 randomly selected commercial airline flights, including 68 smoking flights. When the average RSP and nicotine concentrations for the smoking sections of the smoking flights are corrected for concentrations on non-smoking flights, the ratio of RSP to nicotine is about 8 . Ventilation rates on the planes were very high and often there was no recirculation of air.

The ratios of particulate matter to nicotine show very little variation among cigarette brands or across studies. The ratios that we measured were only slightly lower than those measured in other chamber studies. This may have been due to differences in chamber protocols and the "age" of the ETS. In the other chamber studies, cigarettes were continuously smoked by subjects during the sampling, while in our study three cigarettes were machine smoked prior to initiation of sampling. As noted above, our ETS particulate emission factors were low relative to other chamber studies. Therefore, our ratios of particulate matter to nicotine are also low.

Despite the wide ranges in concentrations and conditions in the 96 residences, the ratios of particulate matter to nicotine showed relatively little variation. The concentrations of particulate matter and nicotine from smoking in buildings are determined by a number of factors, including number of cigarettes smoked, the volume of the space, removal by air infiltration and deposition to surfaces, and re-emission from surfaces. This suggests that, as the net result of these processes, particulate matter and nicotine are behaving similarly.

In general, the chamber studies and field study taken together support the use of the ratio of particulate matter to nicotine for first-order approximations of the contributions of ETS to particulate matter in residences and probably other types of buildings.

\subsubsection{NO, NOx and CO}

$\mathrm{NO}, \mathrm{NO}_{\mathrm{x}}$ and $\mathrm{CO}$ levels were continuously monitored during the ETS experiments. Measurements were initiated prior to cigarette smoking to determine background levels, which were very low. Concentrations reached maximum or peak levels shortly after completion of smoking and remained close to this level throughout the experiments. Table 6.23 summarizes the average $\mathrm{CO}$ and NO chamber concentrations measured in each experiment and the emission factors calculated from those concentrations. 
Table 6.23. Environmental chamber concentrations of carbon monoxide and nitric oxide from environmental tobacco smoke during experiments with six commercial cigarettes and Kentucky reference cigarette 1R4F.

\begin{tabular}{|c|c|c|c|c|c|}
\hline Cigarette & $\begin{array}{c}\text { Carbon } \\
\text { Monoxide, } \\
\text { ppm }^{2}\end{array}$ & $\begin{array}{l}\text { Nitric Oxide } \\
\text { Peak, } \\
\text { ppb }^{2}\end{array}$ & $\begin{array}{c}\text { Nitric Oxide } \\
\text { after } 250 \text { min, } \\
\text { ppb }^{\text {b. }}\end{array}$ & $\begin{array}{c}\text { Carbon Monoxide } \\
\text { Emission Factor, } \\
\text { mg/cig }\end{array}$ & $\begin{array}{l}\text { NO Emission } \\
\text { Factor, } \\
\text { mg/cig }\end{array}$ \\
\hline $\mathbf{A}$ & 13 & 110 & 102 & 99 & 0.90 \\
\hline $\mathrm{A}^{\mathrm{c}}$ & 13 & 119 & 106 & 99 & 0.97 \\
\hline B & 12 & 109 & 96.0 & 92 & 0.89 \\
\hline C & 7 & 76.8 & 65.5 & 54 & 0.63 \\
\hline $\mathbf{D}$ & 9 & 113 & 93.9 & 69 & 0.93 \\
\hline $\mathbf{E}$ & 18 & 138 & 106 & 139 & 1.13 \\
\hline $\mathbf{F}$ & 16 & 103 & 85.2 & 122 & 0.84 \\
\hline 1R4F & 12 & 107 & 91.2 & 92 & 0.87 \\
\hline Average $^{\text {d. }}$ & 12 & 109 & 91.7 & 96 & 0.89 \\
\hline Std. Dev. ${ }^{\text {d. }}$ & 4.1 & 19.8 & $\overline{14.8}$ & 32 & 0.16 \\
\hline C. V.t.,e. & 33 & 18 & 16 & 33 & 18 \\
\hline
\end{tabular}

a. Maximum gas concentration for three cigarettes. Within the uncertainty of the measurements, the $\mathrm{CO}$ peak concentrations did not change over time.

b. Nitric oxide concentration at end of the chamber experiment for three cigarettes.

c. Analysis of a duplicate sample.

d. 1R4F omitted; $n=6$.

e. C. V.: Coefficient of Variation $=($ Std. Dev. X 100)/Average.

Peak $\mathrm{CO}$ levels remained unchanged in the sealed chamber over the course of the experiments within the sensitivity of the $\mathrm{CO}$ monitor. The concentrations of $\mathrm{CO}$ in the chamber over the 4-hour period ranged from 7 to $18 \mathrm{ppm}$ for the eight experiments with an average of $12 \mathrm{ppm}$. For comparison, the current ambient (outdoor) air quality standard for $\mathrm{CO}$ is $9 \mathrm{ppm}$ for an 8-hour exposure. The average emission factor for CO was determined for these experiments to be $96 \pm 32 \mathrm{mg} / \mathrm{cig}$. This value is within the range of previously reported SS emission factors (NRC, 1986).

The $\mathrm{NO}_{\mathrm{x}}$ levels in the chamber were only slightly above the $\mathrm{NO}$ levels. Therefore, the $\mathrm{NO}_{2}$ levels, which are measured with the chemiluminescence monitor as the difference between $\mathrm{NO}_{\mathrm{x}}$ and $\mathrm{NO}$, were only slightly above background laboratory levels. Also, the uncertainty in the calculated $\mathrm{NO}_{2}$ levels was high. Consequently, only NO levels are reported in Table 6.23. Although the NO levels decayed throughout the experimental period, the average difference between the peak and final levels was only $15 \%$. Since air infiltration alone would account for a $12 \%$ decrease in the airborne concentration over the period, the loss of NO can be attributed to air infiltration rather than chemical reactions of NO. These results suggest that there was very little chemical reaction in the chamber to generate $\mathrm{NO}_{2}$ under the conditions of this study. 


\subsection{REFERENCES}

Baker, R.R. and Proctor, C.J. (1990) The origins and properties of environmental tobacco smoke. Environ. Intern., 16: 231-245.

Bell, J.H., Crouse, W.E., Morgan, J.P. and Smart, D.R. (1987) An improved apparatus for the determination of formaldehyde in sidestream smoke. Tobacco Chemists' Research Conference, Paper No. 25.

Benner, C.L., Bayona, J.M., Caka, F.M., Tang, H., Lewis, L., Crawford, J., Lamb, J.D., Lee, M.L., Lewis, E.A., Hansen, L.D. and Eatough, D.J. (1989) Chemical composition of environmental tobacco smoke. 2. particulate-phase compounds. Environ. Sci. Technol., 23: 688-699.

Brunnemann, K.D., Fink, W. and Moser, F. (1980) Analysis of volatile N-nitrosamines in mainstream and sidestream smoke from cigarettes by GLC-TEA. Onocology, 37: 217-222.

Brunnemann, K. D. and Hoffmann, D. (1974) The pH of tobacco smoke. Fd. Cosmet. Toxicol., 12: 115 124.

Brunnemann, K.D. and Hoffmann, D. (1978) Chemical studies of tobacco smoke LIX. Analysis of volatile nitrosamines in tobacco smoke and polluted indoor environments. In: Environmental Aspects of N-Nitroso Compounds (IARC No. 19), E. Walker, L. Griciute, M. Castegnaro and R. Lyle, Eds., International Agency for Research on Cancer, Lyons, France.

Brunnemann, K.D., Kagan, M.R., Cox, J. E. and Hoffmann, D. (1990) Analysis of 1,3-butadiene and other selected gas-phase components in cigarette mainstream and sidestream smoke by gas chromatography-mass selective detection. Carcinogenesis 11(10): 1863-1868.

Brunnemann, K.D., Yu, L. and Hoffmann, D. (1977) Assessment of carcinogenic volatile N-nitrosamines in tobacco and in mainstream and sidestream smoke from cigarettes. Cancer Res., 37: 3218-3222.

Caldwell, W.S. and Conner, J.M. (1990) Artifact formation during smoke trapping: An improved method for determination of N-nitrosamines in cigarette smoke. J. Assoc. Off. Anal. Chem., 73: 783-789. 
CARB (1989) Information on Substances for Review as Toxic Air Contaminants. M. Jones, Principal Ed., Report ARB/SSD/89-01, California Air Resources Board, Stationary Source Division, Sacramento, CA.

CARB (1993) Proposed Identification of Federal Hazardous Air Pollutants as Toxic Air Contaminants. R. Krieger, A. Krichevsky and E. Parkhurst. California Environmental Protection Agency, Air Resources Board, Sacramento, CA.

Chortyk, O.T. and Schlotzhauer, W.S. (1989) The contribution of low tar cigarettes to environmental tobacco smoke. J. Anal. Toxicol., 13: 129-134.

Cucco, J.A. and Brown, P.R. (1981) Confirming the presence of N-nitrosamines in ambient air and cigarette smoke by converting to and photochemically altering their corresponding $\mathrm{N}$-nitrosamines. $J$. Chromatogr. 213: 253-263.

Daisey, J.M., Gadgil, A. and Hodgson, A.T. Model estimates of the contributions of environmental tobacco smoke to volatile organic compound exposures in office buildings. Indoor Air 1: $37-45$ (1991).

Dreyfors, J.M., Jones, S.B. and Sayed, Y. (1989) Hexamethylenetetramine: A review. Am. Ind. Hyg. Assoc. J., 50: 579-585.

Eatough, D.J. (1993) Assessing exposure to environmental tobacco smoke. In: Modeling of Indoor Air Quality and Exposure, ASTM STP 1205, N.L. Nagda, Ed. American Society for Testing and Materials, Philadelphia, pp. 42-63.

Eatough, D.J., Benner, C., Mooney, R.L., Bartholomew, D., Steiner, D.S., Hansen, L.D., Lamb, J.D. and Lewis, E.A. (1986) Gas and particle phase nicotine in environmental tobacco smoke. Paper No. 86-68.5, Proceedings of the 79th Annual Meeting of the Air Pollution Control Association, Minneapolis, MN, June 22-27.

Eatough, D.J., Hansen, L.D. and Lewis, E.A. (1990) "The chemical characterization of environmental tobacco smoke," pp. 3-39 In: Environmental Tobacco Smoke, Proceedings of the International Symposium at McGill University, 1989, D.J. Ecobichon and J.M. Wu, Eds., Lexington Books, D.C. Heath and Co., Lexington. 
Fine, D.H., Rounbehler, D.P., Sawicki, E. and Krost, K. (1977) Determination of dimethylnitrosamine in air and water by thermal energy analysis: Validation of analytical procedures. Environ. Sci. Technol. 11: $577-580$.

Fine, D.H. Rounbehler, D.P. and Goff, U. (1993) Determination of airborne nitrosamines by means of airsampling cartridges and GC-TEA. In: Environmental Carcinogens Methods of Analysis and Exposure Measurement, (IARC No 109), B. Seifert, H.J. van de Wiel, B. Dodet and I.K O'Neill, pp. 269-274.

Fischer, S. and Spiegelhalder, B. (1989) Improved method for the determination of tobacco-specific nitrosamines (TSNA) in tobacco smoke. Beitr. z. Tabakfor. Intern., 14: 145-153. ।

Fischer, S., Spiegelhalder, B. and Preussmann, R. (1989) Tobacco-specific nitrosamines in mainstream smoke of West German cigarettes - tar alone is not a sufficient index for the carcinogenic potential of cigarette smoke. Carcinogenesis, 10: 169-173.

Fung, K. and Grosjean, D. (1981) Determination of nanogram amounts of carbonyls as 2,4dinitrophenylhydrazones by high-performance liquid chromatography. Anal. Chem., 53: 168-171.

FTC (1992) Federal Trade Commission. Tar, Nicotine and Carbon Monoxide of the Smoke of 543 Varieties of Domestic Cigarettes.

Grosjean, D. (1992) Atmospheric concentrations and temporal variations of $C_{3}-C_{3}$ carbonyl compounds at two rural sites in central Ontario. Atmos. Environ., 26A: 349-351.

Guerin, M.R., Higgins, C.E and Jenkins, R.A. (1987) Measuring environmental emissions from tobacco combustion: Sidestream cigarette smoke literature review. Atmospheric Environ, 21(2): 291-297.

Hammond, S.K., Leaderer, B.P., Roche, A.C. and Schenker, M. (1987) Collection and analysis of nicotine as a marker for environmental tobacco smoke. Atmos. Environ., 21: 457-462.

Hecht, S.S., Adams, J.D. and Hoffmann, D. (1983) In: Environmental Carcinogens Selected Methods of - Analysis, N-Nitroso Compounds, H. Egan, R. Preussmann, I.K. O'Neill, G. Eisenbrand, B. Spiegelhalder and H. Bartsch (Eds), Scientific Publication No. 45, IARC, Lyon, France, pp. 93101. 
Heller, S.R. and Milne, G.W.A. (1978) EPANIH Mass Spectral Data Base, U.S. National Bureau of Standards Reference Data Ser., 63, U.S. Government Printing Office, Washington, D.C.

Hodgson, A.T. and Girman, J.R. (1989) Application of a multisorbent sampling technique for investigations of volatile organic compounds in buildings. In: Design and Protocol for Monitoring Indoor Air Quality, ASTM STP 1002, N.L. Nagda, J.P. and Harper (Eds.) American Society for Testing and Materials, Philadelphia, PA.

Hodgson, A.T. and Wooley, J.D. (1991) Assessment of Indoor Concentrations, Indoor Sources and Source Emissions of Selected Volatile Organic Compounds, Final Report. Interagency Agreement A933063 between the California Air Resources Board and Lawrence Berkeley Laboratory, Berkeley, CA.

Hodgson, A.T., Wooley, J.D. and Daisey, J.M. (1993) Emissions of volatile organic compounds from new carpets measured in a large-scale environmental chamber. J. Air Waste Manage. Assoc., 43: 316324.

Hoffmann, D. and Wynder, E.L. (1986) Constituents and bioactivity of tobacco smoke. In: Tobacco: A Major International Health Hazard, D.G. Zaridze and R. Peto, Eds., IARC Scientific Publications, Vol. 74, Lyon, France.

IARC (1978). IARC Monographs on the Evaluation of the Carcinogenic Risk of Chemicals to Humans: Some N-nitroso Compounds. Vol. 17. International Agency for Research on Cancer. Lyon, France.

ISO (International Organization of Standardization) (1986) ISO 3308-1986 (E) International Standard: Cigarettes - Routine Analytical Cigarette Smoking Conditions - Definitions and Standard Conditions. Beuth Verlag, Berlin.

Jenkins, P.L., Phillips, T.J., Mulberg, E.J. and Hui, S.P. (1992) Activity patterns of Californians: Use of and proximity to indoor pollutant sources. Atmos. Environ., 26A: 2141-2148.

Jermini, C., Weber, A. and Grandjean, E. (1976) Quantitative Bestimmung verschiedener Gasphasekomponenten des Nebenstromrauches von Zigaretten in der Raumluft als Beitrag zum Problem des Passivrauchens. Intern. Arch. Occup. Environ. Health, 36: 169-181. 
Kuwata, K., Uebori, M. and Yamasaki, Y. (1979) Determination of aliphatic and aromatic aldehydes in polluted airs as their 2,4-dinitrophenylhydrazones by high performance liquid chromatography. $J$. Chromatogr. Sci., 17: 264-268.

Kuwata, K., Uebori, M., Yamasaki, H. and Kuge, Y. (1983) Determination of aliphatic aldehydes by liquid chromatography. Anal. Chem., 55: 2013-2016.

Leaderer, B.P. and Hammond, S.K. (1991) Evaluation of vapor-phase nicotine and respirable suspended particle mass as markers for environmental tobacco smoke. Environ. Sci. Technol., 25: 770-777.

Lofroth, G., Burton, R.M., Forehand, L., Hammond, K., Seila, R.L., Zweidinger, R.B. and Lewtas, J. (1989) Characterization of Environmental Tobacco Smoke. Environ. Sci. Technol., 23: 610-614.

Miesner, E.A., Rudnick, S.N., Hu, F-C, Spengler, J.D., Preller, L., Ozkaynak, H. and Nelson, W. (1989) Particulate and nicotine sampling in public facilities and offices. JAPCA, 39: 1577-1582.

Mirvish, S.S., Wallcave, L., Eagen, M. and Shubik, P. (1972) Ascorbate-nitrite reaction: Possible means of blocking the formation of carcinogenic N-nitroso compounds. Science 177: 65-68.

Nagda, N.L., Koonz, M.D., Konheim, A.G. and Hammond, S.K. (1992) Measurement of cabin air quality aboard commmercial airliners. Atmospheric Environment 26A: 2203-2210.

NRC, National Research Council (1986) Environmental Tobacco Smoke. Measuring Exposures and Assessing Health Effects. National Academy Press, Washington, D.C.

Nelson, P.R., Heavener, D.L., Collie, B.B., Maiolo, K.C. and Ogden, M.W. (1992) Effect of ventilation and sampling time on environmental tobacco smoke component ratios. Environ. Sci. Technol., 26: 1909-1915.

Neurath, G. and Emhke, H. (1964) Apparatur zur Untersuchung des Nebenstromrauches. Beitr. Tabakforsch., 2: 117-121.

Offermann, F.J., Sextro, R.G., Fisk, W.J., Grimsrud, D.T., Nazaroff, W.W., Nero, A.V., Revzan, K.L. and Yater, J. (1985) Control of respirable particles in indoor air with portable air cleaners. Atmos. Environ., 19: 1761-1771. 
Ogden, M.W. (1988) High resolution gas chromatography of tobacco smoke: the contributions of Kurt Grob. High Resolut Chromatogr., Gas Chromatogr., 11: 428-440.

Ogden, M.W., Eudy, L.W., Heavner, D.L., Conrad, Jr., F.W. and Green, C.R. (1989) Improved gas chromatographic determination of nicotine in environmental tobacco smoke. Analyst, 114: 10051008.

Pierce, J.P., Gilpin, E., Burns, D.M., Whalen, E., Rosbrook, B., Shopland D. and Johnson, M. (1991) Does tobacco advertising target young people to start smoking? J. Amer. Med. Assoc., 266: 3154-3158.

Pierce, J.P., J. Goodman, E. Gilpin and C. Berry. (1992) Technical Report on Analytical Methods and Approaches Used in the Tobacco Use in California, 1990-1991, Report. Sacramento: California Department of Health Services,

Preussmann, R. (1984) N-nitroso compounds: Occurrence, biological effects and relevance to human cancer. I.K. O'Neil, R.C. Van Borstel, C.T. Miller, J. Lang and H. Bartsch (Eds.), pp. 3-15, IARC Scientific Publication No. 57, International Agency for Research on Cancer, Lyon.

R.J. Reynolds Tobacco Co. (1988) New cigarette prototypes that heat instead of burn tobacco. WinstonSalem, NC, R.J. Reynolds Tobacco Co.

Rickert, W.S., Robinson, J.C. and Collishaw, N. (1984) Yields of tar, nicotine and carbon monoxide in the sidestream smoke from 15 brands of Canadian cigarettes. Am. J. Pub. Health, 74: 228-231.

Riggin, R.M. (1984) Compendium of Methods for the Determination of Toxic Organic Compounds in Ambient Air. U.S. EPA Report EPA-600/4-84-041, Environmental Monitoring Systems Laboratory, Research Triangle Park, NC.

Risner, C.H. and Martin, P. (1994) Quantitation of formaldehyde, acetaldehyde and acetone in sidestream cigarette smoke by high-performance liquid chromatography. J. Chromatogr. Sci., 32: 76-82.

Schlitt, H. and Knoppel, H. (1989) Carbonyl compounds in mainstream and sidestream cigarette smoke. In: Present and Future of Indoor Air Quality, Proceedings of the Brussels Conference, 14-16 February 1989, Exerpta Medica, Amsterdam, pp. 197-206. 
Schmeltz, I. and Hoffmann, D. (1977) Nitrogen-containing compounds in tobacco and tobacco smoke. Chem. Rev., 77(3): 295-311.

Shoemaker, D.P., Garland, C.W. and Steinfeld, J.I. (1974) Experiments in Physical Chemistry, Third Edition. McGraw-Hill, Inc., New York, pp. 725.

Thermedics, Inc., Analytical Instruments Division, Watham, MA. "Thermosorb/N air sampler: Nitrosamine Air Sampling System Brochure.”

U.S. EPA (1992) U.S. Environmental Protection Agency. Respiratory Health Effects of Passive Smoking: Lung Cancer and Other Disorders. EPA/600/6-90/006F

Vilcins, G. and Lephardt, J.P. (1975) Aging process of cigarette smoke: Formation of methyl nitrite. Chem. Ind. (London), 22: 974-975.

Wiley, J.A., Robinson, J.P., Piazza, T., Garrett, K., Cirksena, K., Cheng, Y.-T. and Martin, G. (1991) Activity Patterns of California Residents. Final Report, Contract No. A6-177-33, California Air Resources Board, Research Division, Sacramento, CA.

Xu, M., Nematollahi, M., Sextro, R.G., Gadgil, A.J. and Nazaroff, W.W. (1994) Deposition of tobacco smoke particles in a low ventilation room. Aero. Sci. Technol., 20: 194-206. 


\subsection{GLOSSARY OF TERMS}

\begin{tabular}{|c|c|}
\hline Acronym & \\
\hline ach & Air changes per hour \\
\hline CO & Carbon monoxide \\
\hline C.V. & Coefficient of variation \\
\hline DCM & Dichloromethane \\
\hline DNPH & 2,4-dinitrophenylhydrazine \\
\hline DLX & Deluxe (cigarettes) \\
\hline EA & Ethyl acetate \\
\hline ETS & Environmental tobacco smoke \\
\hline GC & Gas chromatograph \\
\hline GC/MS & Gas chromatograph/mass spectrometer \\
\hline GC/TEA & Gas chromatograph/thermal energy analyzer \\
\hline HPLC & High performance liquid chromatograph \\
\hline $\mathbf{L T}$ & Light (Cigarettes) \\
\hline MEN & Mentholated (cigarettes) \\
\hline MSD & Mass selective detector \\
\hline NF & Non-filtered (cigarettes) \\
\hline NDMA & N-Nitrosodimethylamine \\
\hline NDEA & N-Nitrosodiethylamine \\
\hline NMPH & N-Nitrosomorpholine \\
\hline No & Nitric oxide \\
\hline $\mathrm{NO}_{2}$ & Nitrogen dioxide \\
\hline $\mathrm{NO}_{\mathbf{x}}$ & Oxides of nitrogen \\
\hline NPYR & N-Nitrosopyrrolidine \\
\hline $\mathbf{P M}$ & Particulate matter \\
\hline REG & Regular (cigarettes) \\
\hline RSP & Respirable particulate matter \\
\hline SS & Sidestream smoke \\
\hline SSA & Sidestream sampling apparatus \\
\hline Std. Dev. & Standard deviation of the mean \\
\hline TCGF & Teflon-coated glass-fiber (filters) \\
\hline TEA & Thermal energy analyzer \\
\hline ULT-LT & Ultra light (cigarettes) \\
\hline VNA & Volatile N-nitrosamines \\
\hline VOCs & Volatile organic compounds \\
\hline
\end{tabular}




\begin{abstract}
APPENDIX
This appendix presents the concentrations in $\mu \mathrm{g} \mathrm{m}^{-3}$ for the 15 volatile organic compounds measured in the ETS experiments conducted in the environmental chamber. The results for experiments with eight cigarettes are given. The concentrations are shown for the 0-1, 1-2, 2-3, and 3-4 hour sampling periods. These data are statistically summarized. These values were used to calculate the ETS emission factors.
\end{abstract}


Cigarette A

\begin{tabular}{|l|c|c|c|c|c|c|c|}
\hline & \multicolumn{4}{|c|}{ Sampling Time } & \multicolumn{3}{c|}{ Statistical Data } \\
\hline Compound & $\mathbf{0 - 1} \mathbf{~ h r}$ & $\mathbf{1 - 2} \mathbf{~ h r}$ & $\mathbf{2 - 3} \mathbf{~ h r}$ & $\mathbf{3 - 4} \mathbf{~ h r}$ & Average & Std. Dev. & C.V., ${ }^{\text {a }}$. \\
\hline Acrylonitrile & $*$ & $\mathbf{1 7 . 5}$ & 16.1 & 15.4 & 16.3 & 1.09 & 7 \\
\hline 1,3-Butadiene & $*$ & 26.8 & 31.4 & 28.4 & 28.9 & 2.29 & 8 \\
\hline 2-Butanone & $*$ & 35.3 & 38.5 & 36.8 & 36.9 & 1.61 & 4 \\
\hline Benzene & 36.0 & 72.6 & 59.8 & 62.4 & 57.7 & 15.5 & 27 \\
\hline m,p-Cresol & $*$ & 9.84 & 8.95 & 7.07 & 8.60 & 1.42 & 16 \\
\hline o-Cresol & $*$ & 4.40 & 3.54 & 2.77 & 3.60 & 0.81 & 23 \\
\hline Ethyl benzene & 15.1 & 16.2 & 15.4 & 13.9 & 15.1 & 0.93 & 6 \\
\hline Phenol & $*$ & 41.8 & 40.8 & 25.8 & 36.1 & 8.95 & 25 \\
\hline Pyridine & 66.1 & 76.9 & 73.8 & 69.8 & 71.7 & 4.70 & 7 \\
\hline Pyrrole & 60.3 & 69.0 & 64.8 & 57.3 & 62.8 & 5.15 & 8 \\
\hline Styrene & 22.5 & 23.7 & 22.6 & 21.5 & 22.6 & 0.93 & 4 \\
\hline Toluene & 99.3 & 108 & 96.9 & 90.9 & 98.9 & 7.29 & 7 \\
\hline 3-Vinylpyridine & $*$ & 98.3 & 88.0 & 77.0 & 87.8 & 10.6 & 12 \\
\hline m,p-Xylene & 44.0 & 47.9 & 45.0 & 42.5 & 44.9 & 2.26 & 5 \\
\hline o-Xylene & 9.06 & 10.1 & 9.88 & 9.11 & 9.5 & 0.52 & 5 \\
\hline
\end{tabular}

* water in the sampling tube

a. C.V.: $=$ Coefficient of Variation $=($ Std. Dev. $\times 100) /$ Average.

Cigarette A (duplicate)

\begin{tabular}{|l|c|c|c|c|c|c|c|}
\hline & \multicolumn{4}{|c|}{ Sampling Time } & \multicolumn{3}{c|}{ Statistical Data } \\
\hline Compound & $\mathbf{0 - 1} \mathbf{~ h r}$ & $\mathbf{1 - 2} \mathbf{~ h r}$ & $\mathbf{2 - 3} \mathbf{~ h r}$ & $\mathbf{3 - 4} \mathbf{~ r}$ & Average & Std. Dev. & C.V., $\%$. \\
\hline Acrylonitrile & 13.2 & 14.1 & 14.7 & 14.0 & 14.0 & 0.60 & 4 \\
\hline 1,3-Butadiene & 17.0 & 18.9 & 20.5 & 24.0 & 20.1 & 2.98 & 15 \\
\hline 2-Butanone & 33.0 & 37.8 & 39.8 & 38.8 & 37.3 & 3.00 & 8 \\
\hline Benzene & 57.8 & 49.5 & 48.3 & 48.4 & 51.0 & 4.57 & 9 \\
\hline m,p-Cresol & 12.1 & 7.65 & 6.96 & 5.27 & 8.00 & 2.90 & 36 \\
\hline o-Cresol & 5.50 & 3.86 & 3.44 & 1.97 & 3.70 & 1.45 & 39 \\
\hline Ethyl benzene & 13.7 & 13.2 & 12.9 & 12.4 & 13.0 & 0.56 & 4 \\
\hline Phenol & 41.1 & 33.8 & 36.6 & 24.8 & 34.1 & 6.86 & 20 \\
\hline Pyridine & 60.1 & 51.6 & 52.9 & 48.5 & 53.2 & 4.91 & 9 \\
\hline Pyrrole & 59.5 & 57.8 & 55.7 & 53.5 & 56.6 & 2.61 & 5 \\
\hline Styrene & 20.5 & 20.2 & 20.5 & 20.5 & 20.4 & 0.17 & 1 \\
\hline Toluene & 91.3 & 87.9 & 87.9 & 80.9 & 87.0 & 4.40 & 5 \\
\hline 3-Vinylpyridine & 97.1 & 84.3 & 75.6 & 66.9 & 81.0 & 12.9 & 16 \\
\hline m,p-Xylene & 42.8 & 39.3 & 38.1 & 36.7 & 39.2 & 2.61 & 7 \\
\hline o-Xylene & 8.93 & 8.85 & 8.29 & 7.59 & 8.40 & 0.62 & 7 \\
\hline
\end{tabular}

a. C.V.: $=$ Coefficient of Variation $=($ Std. Dev. $x$ 100) $/$ Average 


\section{Cigarette B}

\begin{tabular}{|l|c|c|c|c|c|c|c|}
\hline & \multicolumn{4}{|c|}{ Sampling Time } & \multicolumn{3}{c|}{ Statistical Data } \\
\hline Compound & $\mathbf{0 - 1} \mathbf{~ h r}$ & $\mathbf{1 - 2} \mathbf{~ h r}$ & $\mathbf{2 - 3} \mathbf{~ h r}$ & $\mathbf{3 - 4} \mathbf{~ h r}$ & Average & Std. Dev. & C.V., ${ }^{\text {a \%. }}$ \\
\hline Acrylonitrile & 11.7 & 13.8 & 13.1 & $\mathbf{1 4 . 9}$ & 13.4 & 1.32 & 10 \\
\hline 1,3-Butadiene & 17.7 & 21.1 & 18.4 & 22.0 & 19.8 & 2.09 & 11 \\
\hline 2-Butanone & 31.3 & 36.6 & 37.7 & 40.0 & 36.4 & 3.71 & 10 \\
\hline Benzene & 52.4 & 46.8 & 56.0 & 56.3 & 52.9 & 4.44 & 8 \\
\hline m,p-Cresol & 11.0 & 7.84 & 5.58 & 4.39 & 7.20 & 2.89 & 40 \\
\hline o-Cresol & 4.56 & 3.49 & 2.97 & 1.81 & 3.20 & 1.14 & 36 \\
\hline Ethyl benzene & 13.2 & 13.4 & 12.6 & 12.6 & 13.0 & 0.39 & 3 \\
\hline Phenol & 34.8 & 28.4 & 25.2 & 23.0 & 27.9 & 5.14 & 18 \\
\hline Pyridine & 49.2 & 60.6 & 57.2 & 59.6 & 56.6 & 5.19 & 9 \\
\hline Pyrrole & 51.5 & 51.2 & 51.0 & 52.4 & 51.5 & 0.63 & 1 \\
\hline Styrene & 18.6 & 19.3 & 17.7 & 19.0 & 18.6 & 0.73 & 4 \\
\hline Toluene & 84.9 & 85.6 & 83.3 & 83.6 & 84.3 & 1.10 & 1 \\
\hline 3-Vinylpyridine & 88.5 & 78.6 & 66.7 & 62.3 & 74.0 & 11.9 & 16 \\
\hline m,p-Xylene & 40.9 & 40.9 & 38.0 & 38.2 & 39.5 & 1.62 & 4 \\
\hline o-Xylene & 9.58 & 9.24 & 8.63 & 8.75 & 9.10 & 0.44 & 5 \\
\hline
\end{tabular}

a. C.V.: $=$ Coefficient of Variation $=($ Std. Dev. $x$ 100) $/$ Average.

\section{Cigarette C}

\begin{tabular}{|l|c|c|c|c|c|c|c|}
\hline & \multicolumn{4}{|c|}{ Sampling Time } & \multicolumn{3}{c|}{ Statistical Data } \\
\hline Compound & $\mathbf{0 - 1 ~ h r}$ & $\mathbf{1 - 2} \mathbf{~ h r}$ & $\mathbf{2 - 3} \mathbf{~ h r}$ & $\mathbf{3 - 4} \mathbf{~ h r}$ & Average & Std. Dev. & C.V., \%. \\
\hline Acrylonitrile & 9.38 & 11.6 & 11.6 & 9.28 & 10.5 & 1.32 & 13 \\
\hline 1,3-Butadiene & 6.98 & 23.8 & 21.2 & 13.8 & 16.4 & 7.62 & 46 \\
\hline 2-Butanone & 31.4 & 36.3 & 34.1 & 34.5 & 34.1 & 2.05 & 6 \\
\hline Benzene & 46.5 & 50.8 & 41.6 & 44.9 & 46.0 & 3.82 & 8 \\
\hline m,p-Cresol & 6.45 & 5.32 & 5.23 & 2.80 & 4.95 & 1.54 & 31 \\
\hline o-Cresol & 2.90 & 2.98 & 1.78 & 0.63 & 2.07 & 1.11 & 53 \\
\hline Ethyl benzene & 13.1 & 12.3 & 10.4 & 10.1 & 11.5 & 1.45 & 13 \\
\hline Phenol & 29.3 & 20.4 & 14.8 & 12.3 & 19.2 & 7.53 & 39 \\
\hline Pyridine & 53.4 & 56.8 & 47.1 & 40.5 & 49.4 & 7.21 & 15 \\
\hline Pyrrole & 55.0 & 49.1 & 49.5 & 48.9 & 50.6 & 2.92 & 6 \\
\hline Styrene & 18.6 & 18.4 & 16.8 & 15.4 & 17.3 & 1.52 & 9 \\
\hline Toluene & 84.9 & 89.9 & 79.1 & 68.6 & 80.6 & 9.16 & 11 \\
\hline 3-Vinylpyridine & 72.1 & 70.5 & 53.3 & 47.8 & 60.9 & 12.2 & 20 \\
\hline m,p-Xylene & 41.6 & 39.6 & 32.8 & 32.4 & 36.6 & 4.72 & 13 \\
\hline o-Xylene & 8.14 & 8.63 & 6.58 & 6.74 & 7.52 & 1.02 & 13 \\
\hline
\end{tabular}

a. C.V.: $=$ Coefficient of Variation $=($ Std. Dev. $\times 100) /$ Average. 
Cigarette D

\begin{tabular}{|l|c|c|c|c|c|c|c|}
\hline & \multicolumn{4}{|c|}{ Sampling Time } & \multicolumn{3}{c|}{ Statistical Data } \\
\hline Compound & $\mathbf{0 - 1} \mathbf{~ h r}$ & $\mathbf{1 - 2} \mathbf{~ h r}$ & $\mathbf{2 - 3} \mathbf{~ h r}$ & $\mathbf{3 - 4} \mathbf{~ h r}$ & Average & Std. Dev. & C.V., $\mathbf{q}^{\mathbf{2}}$. \\
\hline Acrylonitrile & 11.2 & 11.1 & 13.1 & 12.7 & 12.0 & 1.02 & 9 \\
\hline 1,3-Butadiene & 15.5 & 18.9 & 18.2 & 19.8 & 18.1 & 1.87 & 10 \\
\hline 2-Butanone & 31.6 & 38.8 & 40.2 & 41.9 & 38.2 & 4.52 & 12 \\
\hline Benzene & 50.3 & 53.4 & 56.1 & 56.4 & 54.1 & 2.86 & 5 \\
\hline m,p-Cresol & 8.62 & 6.79 & 5.49 & 4.79 & 6.40 & 1.68 & 26 \\
\hline o-Cresol & 4.56 & 3.32 & 2.67 & 2.44 & 3.20 & 0.95 & 29 \\
\hline Ethyl benzene & 12.3 & 12.1 & 12.3 & 11.8 & 12.1 & 0.23 & 2 \\
\hline Phenol & 33.6 & 25.3 & 22.7 & 26.4 & 27.0 & 4.70 & 17 \\
\hline Pyridine & 39.1 & 48.1 & 58.7 & 45.8 & 48.0 & 8.14 & 17 \\
\hline Pyrrole & 44.8 & 44.7 & 52.7 & 43.3 & 46.4 & 4.28 & 9 \\
\hline Styrene & 18.8 & 18.7 & 19.5 & 19.6 & 19.2 & 0.44 & 2 \\
\hline Toluene & 83.8 & 80.5 & 91.4 & 79.9 & 83.9 & 5.27 & 6 \\
\hline 3-Vinylpyridine & 64.3 & 62.3 & 56.2 & 50.2 & 58.2 & 6.38 & 11 \\
\hline m,p-Xylene & 37.5 & 36.1 & 37.4 & 35.9 & 36.7 & 0.85 & 2 \\
\hline o-Xylene & 8.25 & 7.98 & 8.11 & 7.82 & 8.00 & 0.18 & 2 \\
\hline
\end{tabular}

a. C.V.: $=$ Coefficient of Variation $=($ Std. Dev. $\times 100) /$ Average.

Cigarette E

\begin{tabular}{|l|c|c|c|c|c|c|c|}
\hline & \multicolumn{4}{|c|}{ Sampling Time } & \multicolumn{3}{c|}{ Statistical Data } \\
\hline Compound & $\mathbf{0 - 1} \mathbf{~ h r}$ & $\mathbf{1 - 2} \mathbf{~ h r}$ & $\mathbf{2 - 3} \mathbf{~ h r}$ & $\mathbf{3 - 4} \mathbf{~ h r}$ & Average & Std. Dev. & C.V., \\
\hline Acrylonitrile & 13.8 & $\mathbf{1 7 . 9}$ & 18.4 & 17.5 & 16.9 & 2.11 & 13 \\
\hline 1,3-Butadiene & 12.1 & 28.2 & 29.4 & 31.1 & 25.2 & 8.80 & 35 \\
\hline 2-Butanone & 45.9 & 56.8 & 57.3 & 58.8 & 54.7 & 5.90 & 11 \\
\hline Benzene & 69.5 & 76.8 & 77.0 & 76.2 & 74.9 & 3.62 & 5 \\
\hline m,p-Cresol & 16.6 & 9.57 & 7.98 & 5.15 & 9.80 & 4.85 & 49 \\
\hline o-Cresol & 5.21 & 3.44 & 3.01 & 2.21 & 3.50 & 1.27 & 37 \\
\hline Ethyl benzene & 18.1 & 21.1 & 18.0 & 20.1 & 19.3 & 1.54 & 8 \\
\hline Phenol & 52.2 & 36.5 & 29.6 & 28.1 & 36.6 & 11.0 & 30 \\
\hline Pyridine & 88.9 & 87.9 & 99.0 & 100 & 94.0 & 6.48 & 7 \\
\hline Pyrrole & 74.0 & 85.8 & 79.0 & 85.8 & 81.1 & 5.74 & 7 \\
\hline Styrene & 26.1 & 29.4 & 24.6 & 27.9 & 27.0 & 2.12 & 8 \\
\hline Toluene & 111 & 128 & 124 & 123 & 122 & 7.16 & 6 \\
\hline 3-Vinylpyridine & 128 & 108 & 99.0 & 87.5 & 106 & 17.0 & 16 \\
\hline m,p-Xylene & 54.3 & 59.4 & 53.2 & 57.3 & 56.1 & 2.84 & 5 \\
\hline o-Xylene & 13.1 & 15.5 & 12.7 & 14.1 & 13.8 & 1.26 & 9 \\
\hline
\end{tabular}

a. C.V.: $=$ Coefficient of Variation $=($ Std. Dev. $x$ 100 $) /$ Average. 
Cigarette F

\begin{tabular}{|l|c|c|c|c|c|c|c|}
\hline & \multicolumn{4}{|c|}{ Sampling Time } & \multicolumn{3}{c|}{ Statistical Data } \\
\hline Compound & $\mathbf{0 - 1} \mathbf{~ h r}$ & $\mathbf{1 - 2} \mathbf{~ h r}$ & $\mathbf{2 - 3} \mathbf{~ h r}$ & $\mathbf{3 - 4} \mathbf{~ h r}$ & Average & Std. Dev. & C.V., \\
\hline Acrylonitrile & 15.9 & 16.2 & 16.9 & 15.7 & 16.2 & 0.50 & 3 \\
\hline 1,3-Butadiene & 21.9 & 30.2 & 25.3 & 19.0 & 24.1 & 4.83 & 20 \\
\hline 2-Butanone & 40.7 & 46.7 & 48.2 & 48.5 & 46.0 & 3.65 & 8 \\
\hline Benzene & 56.7 & 67.3 & 67.3 & 58.1 & 62.3 & 5.74 & 9 \\
\hline m,p-Cresol & 12.4 & 8.28 & 6.37 & 4.93 & 8.00 & 3.25 & 41 \\
\hline o-Cresol & 4.26 & 2.62 & 2.14 & 1.93 & 2.70 & 1.06 & 39 \\
\hline Ethyl benzene & 15.2 & 15.2 & 14.8 & 14.5 & 14.9 & 0.38 & 3 \\
\hline Phenol & 43.1 & 31.0 & 25.5 & 25.5 & 31.3 & 8.26 & 26 \\
\hline Pyridine & 43.4 & 50.9 & 53.7 & 61.8 & 52.4 & 7.58 & 14 \\
\hline Pyrrole & 54.0 & 52.1 & 50.9 & 51.2 & 52.1 & 1.42 & 3 \\
\hline Styrene & 21.8 & 21.9 & 21.5 & 20.5 & 21.4 & 0.68 & 3 \\
\hline Toluene & 94.0 & 94.6 & 94.8 & 91.9 & 93.8 & 1.31 & 1 \\
\hline 3-Vinylpyridine & 102 & 90.7 & 82.9 & 71.9 & 86.9 & 12.8 & 15 \\
\hline m,p-Xylene & 45.4 & 44.4 & 43.1 & 41.9 & 43.7 & 1.52 & 3 \\
\hline o-Xylene & 10.1 & 9.59 & 9.34 & 8.87 & 9.50 & 0.52 & 6 \\
\hline
\end{tabular}

a. C.V.: $=$ Coefficient of Variation $=($ Std. Dev. $\times 100) /$ Average.

Cigarette 1R4F

\begin{tabular}{|l|c|c|c|c|c|c|c|}
\hline & \multicolumn{4}{|c|}{ Sampling Time } & \multicolumn{3}{c|}{ Statistical Data } \\
\hline Compound & $\mathbf{0 - 1} \mathbf{~ h r}$ & $\mathbf{1 - 2} \mathbf{~ h r}$ & $\mathbf{2 - 3} \mathbf{~ h r}$ & $\mathbf{3 - 4} \mathbf{~ h r}$ & Average & Std. Dev. & C.V., ${ }^{\mathbf{2}} \% \mathbf{~}$ \\
\hline Acrylonitrile & 15.5 & 15.9 & 16.1 & 20.2 & 16.9 & 2.20 & 13 \\
\hline 1,3-Butadiene & 19.2 & 29.3 & 17.7 & 34.7 & 25.2 & $\mathbf{8 . 1 4}$ & 32 \\
\hline 2-Butanone & 49.2 & 53.3 & 54.7 & 57.0 & 53.5 & 3.28 & 6 \\
\hline Benzene & 58.7 & 59.5 & 61.1 & 60.1 & 59.8 & 0.99 & 2 \\
\hline m,p-Cresol & 10.2 & 6.55 & 4.14 & 4.88 & 6.40 & 2.70 & 42 \\
\hline o-Cresol & 6.06 & 2.51 & 1.55 & 1.83 & 3.00 & 2.09 & 70 \\
\hline Ethyl benzene & 17.3 & 15.6 & 15.7 & 16.6 & 16.3 & 0.77 & 5 \\
\hline Phenol & 33.3 & 27.7 & 23.3 & 20.6 & 26.2 & 5.56 & 21 \\
\hline Pyridine & 73.4 & 85.9 & 90.1 & 111.5 & 90.3 & 15.9 & 18 \\
\hline Pyrrole & 73.5 & 70.2 & 71.9 & 83.3 & 74.7 & 5.85 & 8 \\
\hline Styrene & 25.0 & 22.2 & 20.9 & 23.6 & 22.9 & 1.75 & 8 \\
\hline Toluene & 102 & 104 & 97.8 & 111 & 104 & 5.46 & 5 \\
\hline 3-Vinylpyridine & 99.5 & 94.4 & 85.7 & 83.7 & 90.8 & 7.42 & 8 \\
\hline m,p-Xylene & 49.7 & 44.8 & 44.1 & 46.5 & 46.3 & 2.49 & 5 \\
\hline o-Xylene & 11.0 & 10.6 & 9.94 & 10.7 & 10.6 & 0.45 & 4 \\
\hline
\end{tabular}

a. C.V.: $=$ Coefficient of Variation $=($ Std. Dev. $\times 100) /$ Average. 